\title{
Synthesis and Investigation of the Abiotic Formation of Pyonitrins
} A-D

\author{
Rahul D. Shingare, Victor Aniebok, Hsiau-Wei Lee and John B. MacMillan \\ Department of Chemistry and Biochemistry, University of California, Santa Cruz, Santa Cruz, \\ CA 95064
}

Supporting Information

Table of contents

1. Methods and materials............................................... S2

2. Figure S1: proposed biosynthesis of pyonitrins A-D production................. S3

3. Experimental details................................................. S3

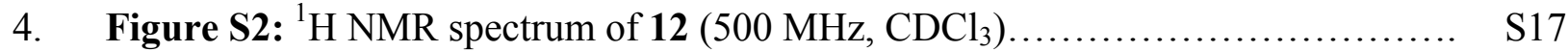

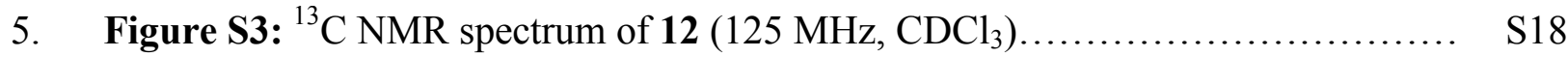

6. Figure S4: ${ }^{1} \mathrm{H}$ NMR spectrum of $13\left(500 \mathrm{MHz}, \mathrm{CDCl}_{3}\right) \ldots \ldots \ldots \ldots \ldots \ldots \ldots \ldots \ldots . . \ldots \ldots$

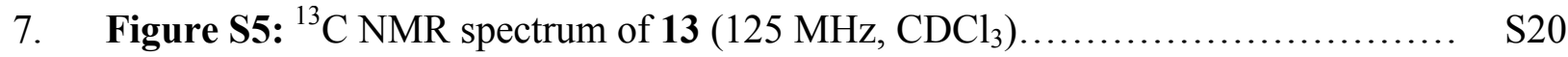

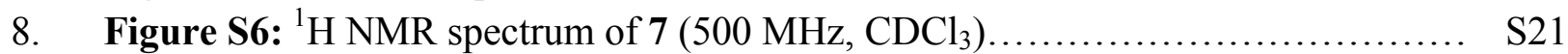

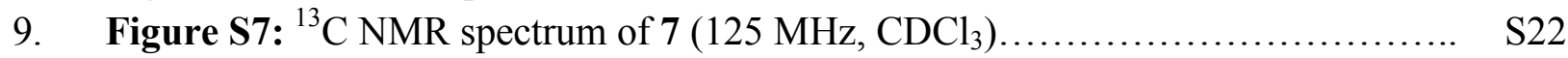

10. Figure S8: ${ }^{1} \mathrm{H}$ NMR spectrum of $18\left(500 \mathrm{MHz}, \mathrm{CDCl}_{3}\right) \ldots \ldots \ldots \ldots \ldots \ldots \ldots \ldots \ldots . . \ldots \ldots \ldots \ldots$

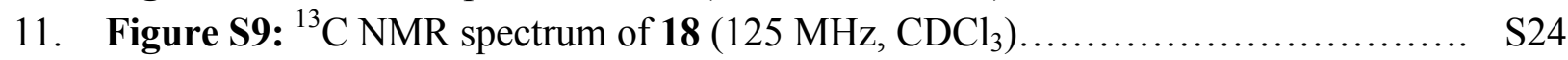

12. Figure S10: ${ }^{1} \mathrm{H}$ NMR spectrum of $22\left(500 \mathrm{MHz}, \mathrm{CDCl}_{3}\right) \ldots \ldots \ldots \ldots \ldots \ldots \ldots \ldots . . . \ldots \ldots \ldots$

13. Figure S11: ${ }^{13} \mathrm{C}$ NMR spectrum of $22\left(125 \mathrm{MHz}, \mathrm{CDCl}_{3}\right) \ldots \ldots \ldots \ldots \ldots \ldots \ldots \ldots . . . \ldots \ldots$

14. Figure S12: ${ }^{1} \mathrm{H}$ NMR spectrum of pyonitrin A $1\left(500 \mathrm{MHz}, \mathrm{DMSO}-d_{6}\right) \ldots \ldots \ldots \ldots . \quad \mathrm{S} 27$

15. Figure S13: ${ }^{13} \mathrm{C}$ NMR spectrum of pyonitrin A $1\left(125 \mathrm{MHz}, \mathrm{DMSO}-d_{6}\right) \ldots \ldots \ldots \ldots . \quad \mathrm{S} 28$

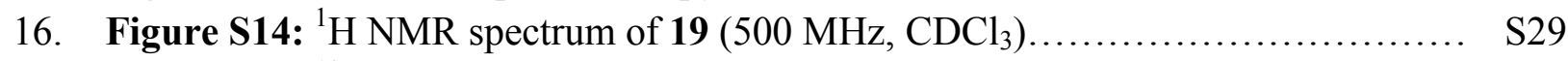

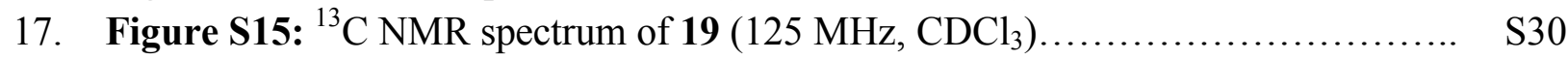

18. Figure S16: ${ }^{1} \mathrm{H}$ NMR spectrum of $23\left(500 \mathrm{MHz}, \mathrm{CDCl}_{3}\right) \ldots \ldots \ldots \ldots \ldots \ldots \ldots \ldots . . \ldots \ldots \ldots \ldots$

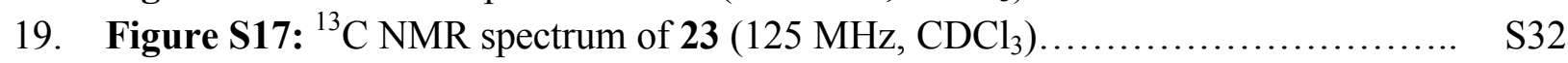

20. Figure S18: ${ }^{1} \mathrm{H}$ NMR spectrum of pyonitrin B $2\left(800 \mathrm{MHz}, \mathrm{DMSO}-d_{6}\right) \ldots \ldots \ldots \ldots . \mathrm{S} 33$

21. Figure S19: ${ }^{13} \mathrm{C}$ NMR spectrum of pyonitrin B $2\left(200 \mathrm{MHz}, \mathrm{DMSO}-d_{6}\right) \ldots \ldots \ldots \ldots . \mathrm{S} 34$

22. Figure S20: ${ }^{1} \mathrm{H}$ NMR spectrum of $\mathbf{2 0}\left(500 \mathrm{MHz}, \mathrm{CDCl}_{3}\right) \ldots \ldots \ldots \ldots \ldots \ldots \ldots \ldots . . . \ldots \ldots$

23. Figure S21: ${ }^{13} \mathrm{C} \mathrm{NMR}$ spectrum of $20\left(125 \mathrm{MHz}, \mathrm{CDCl}_{3}\right) \ldots \ldots \ldots \ldots \ldots \ldots \ldots \ldots . . . \ldots . \ldots . \ldots . \ldots$

24. Figure S22: ${ }^{1} \mathrm{H}$ NMR spectrum of $24\left(500 \mathrm{MHz}, \mathrm{CDCl}_{3}\right) \ldots \ldots \ldots \ldots \ldots \ldots \ldots \ldots . . \ldots \ldots \ldots$

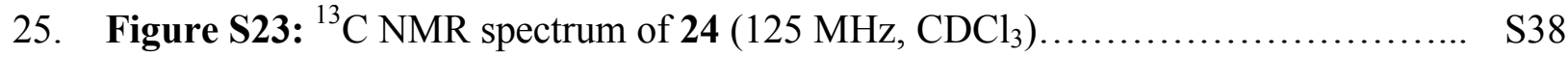

26. Figure S24: ${ }^{1} \mathrm{H}$ NMR spectrum of pyonitrin C $3\left(800 \mathrm{MHz}, \mathrm{DMDO}-d_{6}\right) \ldots \ldots \ldots \ldots . \quad \mathrm{S} 39$

27. Figure S25: ${ }^{13} \mathrm{C}$ NMR spectrum of pyonitrin C $3\left(200 \mathrm{MHz}, \mathrm{DMDO}-d_{6}\right) \ldots \ldots \ldots \ldots . \mathrm{S} 40$

28. Figure S26: ${ }^{1} \mathrm{H}$ NMR spectrum of $21\left(500 \mathrm{MHz}, \mathrm{CDCl}_{3}\right) \ldots \ldots \ldots \ldots \ldots \ldots \ldots \ldots . . . \ldots \ldots 1$ 


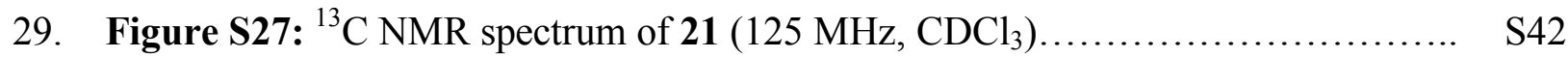

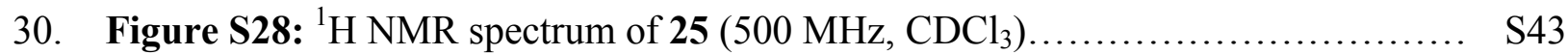

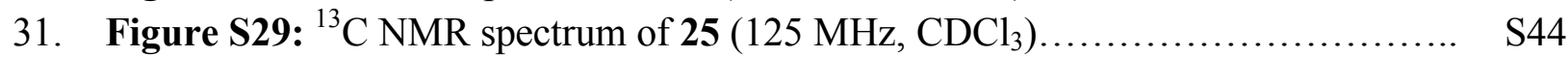

32. Figure S30: ${ }^{1} \mathrm{H}$ NMR spectrum of pyonitrin D $4\left(500 \mathrm{MHz}\right.$, DMSO- $\left.d_{6}\right) \ldots \ldots \ldots \ldots \ldots . . \ldots$ S45

33. Figure S31: ${ }^{13} \mathrm{C}$ NMR spectrum of pyonitrin D $4\left(200 \mathrm{MHz}\right.$, DMSO- $\left.d_{6}\right) \ldots \ldots \ldots \ldots \ldots$ S46

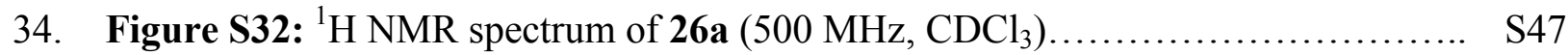

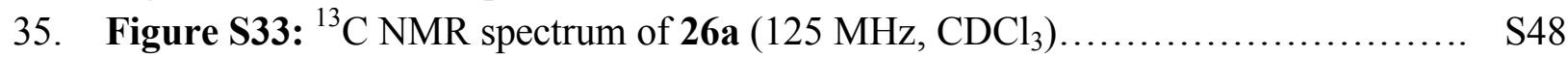

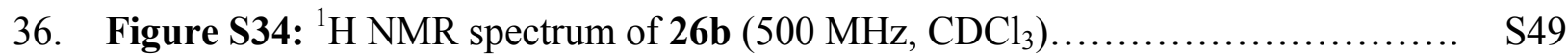

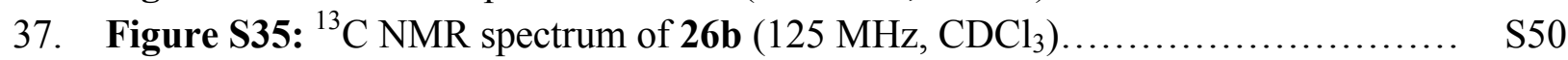

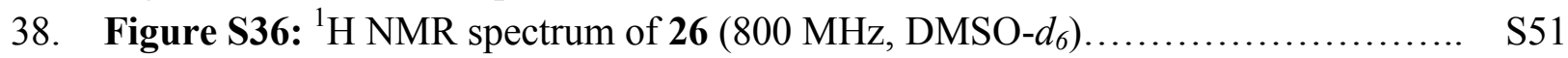

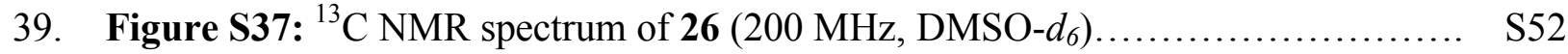

40. Figure S38: ${ }^{1} \mathrm{H}$ NMR spectrum of ${ }^{15} \mathrm{~N}$ pyonitrin A $29\left(500 \mathrm{MHz}\right.$, DMSO- $\left.d_{6}\right) \ldots \ldots \ldots \quad$ S53

41. Figure S39: ${ }^{13} \mathrm{C}$ NMR spectrum of ${ }^{15} \mathrm{~N}$ pyonitrin A $29\left(125 \mathrm{MHz}, \mathrm{DMSO}-d_{6}\right) \ldots \ldots \ldots \quad$ S54

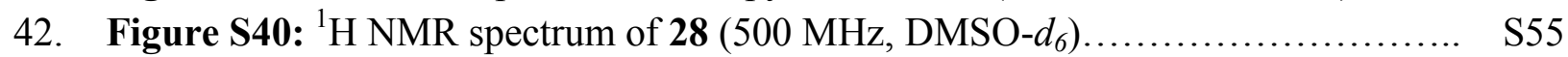

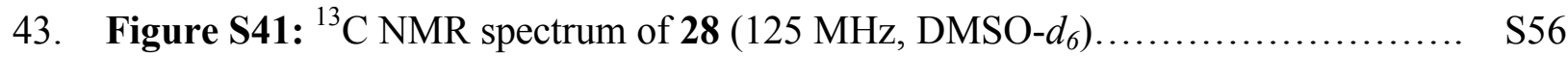

44. Figure S42: ${ }^{1} \mathrm{H}_{-}{ }^{15} \mathrm{~N}$ HMBC NMR spectrum of $\mathbf{2 6}\left(500 \mathrm{MHz}, \mathrm{DMSO}-d_{6}\right) \ldots \ldots \ldots \ldots . \quad \mathrm{S} 57$

45. Figure S43: ${ }^{1} \mathrm{H}-{ }^{15} \mathrm{~N}$ HMBC NMR spectrum of $\mathbf{2 8}\left(500 \mathrm{MHz}\right.$, DMSO- $\left.d_{6}\right) \ldots \ldots \ldots \ldots . \quad$ S58

46. Figure S44: ${ }^{1} \mathrm{H}-{ }^{15} \mathrm{~N}$ HMBC NMR spectrum of $29\left(500 \mathrm{MHz}\right.$, DMSO- $\left.d_{6}\right) \ldots \ldots \ldots \ldots . \quad$ S59

47. Figure S45: Overlay of the ${ }^{1} \mathrm{H}$ NMR of 26, 7, 28 and reaction mixture.............. S60

\section{Methods and materials}

Unless otherwise noted, commercially available materials were used without further purification. Reactions were performed under an atmosphere of nitrogen with magnetic stirring unless noted otherwise. Flash chromatography (FC) was performed using E. Merck silica gel 60 (240-400 mesh). Thin layer chromatography was performed using precoated plates purchased from E. Merck (silica gel $60 \mathrm{PF}_{254}, 0.25 \mathrm{~mm}$ ). Nuclear magnetic resonance (NMR) spectra were recorded on a Bruker Avance III HD $800 \mathrm{MHz}$ and Bruker Avance III HD $500 \mathrm{MHz}$ spectrometer at operating frequencies of $800 / 500 \mathrm{MHz}\left({ }^{1} \mathrm{H}\right.$ NMR) or $200 / 125 \mathrm{MHz}\left({ }^{13} \mathrm{C}\right.$ NMR). Chemical shifts ( $\delta$ ) are given in ppm relative to residual solvent chloroform $\left(\mathrm{CDCl}_{3}:{ }^{1} \mathrm{H}, \delta=7.26 \mathrm{ppm},{ }^{13} \mathrm{C}, \delta=\right.$ $77.16 \mathrm{ppm})$, dimethyl sulfoxide $\left(\left(\mathrm{CD}_{3}\right)_{2} \mathrm{SO}:{ }^{1} \mathrm{H}, \delta=2.50 \mathrm{ppm},{ }^{13} \mathrm{C}, \delta=39.52 \mathrm{ppm}\right)$ and coupling constants $(J)$ in Hz. Multiplicity is tabulated as s for singlet, $\mathrm{d}$ for doublet, $\mathrm{t}$ for triplet, $\mathrm{q}$ for quadruplet, and $\mathrm{m}$ for multiplet and br when the signal in question is broadened. Electrospray ionization mass spectra (ESI-MS) were recorded on a LTQ-Orbitrap Velos Pro MS. Chemicals were purchased from Aldrich, Fisher, Alfa Aesar, TCI, or Oakwood chemicals and used without purification. ${ }^{15} \mathrm{~N}-\mathrm{N}_{2} \mathrm{Na}$ was purchased from Cambridge Isotope Laboratories, Inc. and also used without purification. 
Figure S1: Proposed biosynthesis of pyonitrins A-D production
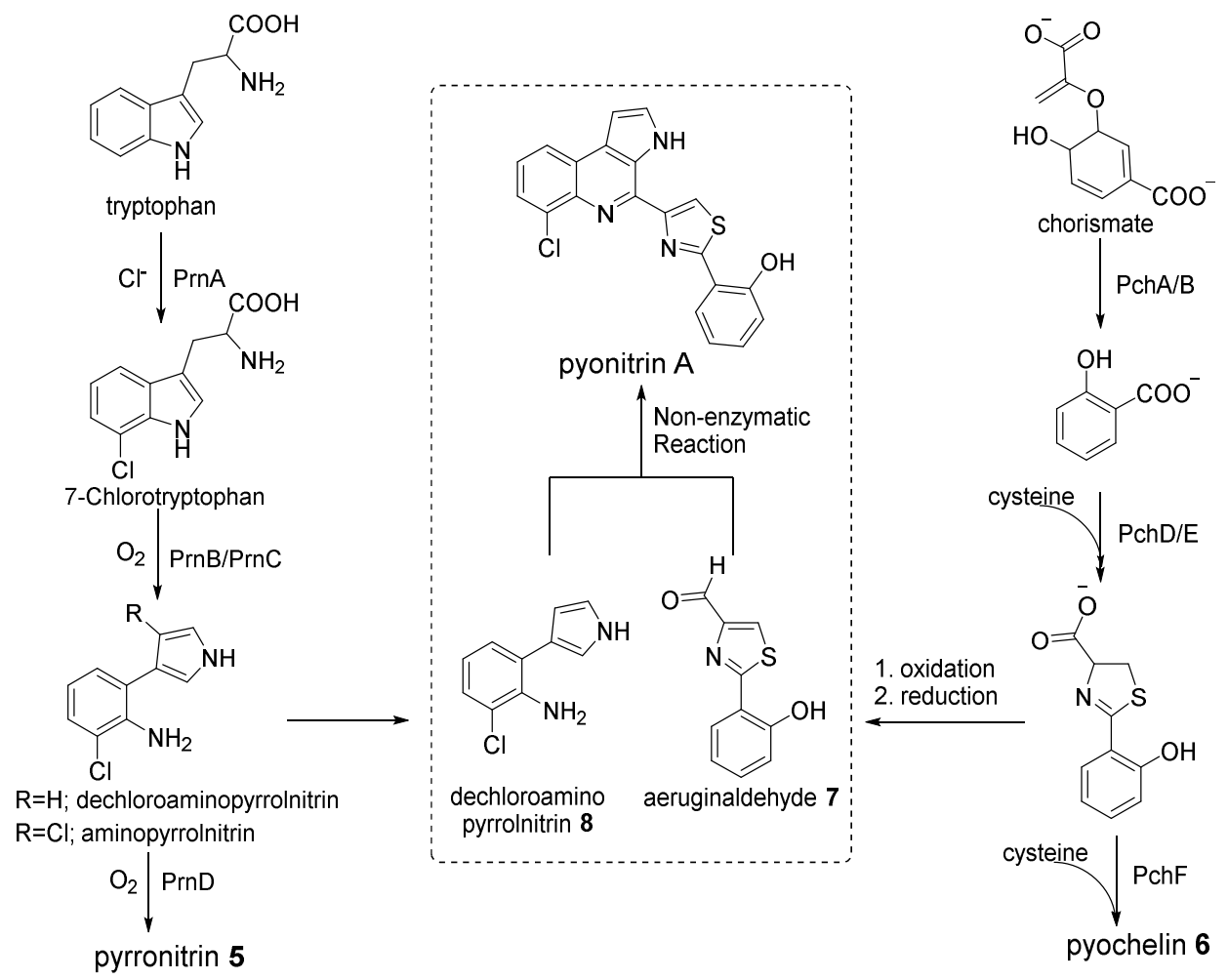

\section{General procedures:}

\section{General procedure 1. Suzuki-Miyaura cross-coupling.}

In an oven-dried round bottom flask, aromatic halide (1.0 equiv), N-(TIPS) pyrrole pinacol boronate (1.2 equiv), palladium acetate $\left[\mathrm{Pd}(\mathrm{OAc})_{2}, 0.05\right.$ equiv], 2-Dicyclohexyl-phosphino-2',6'dimethoxybiphenyl (SPhos, 0.10 equiv), and potassium phosphate $\left(\mathrm{K}_{3} \mathrm{PO}_{4}, 2.0\right.$ equiv) was added under inert atmosphere followed by addition of the solvent system $(2.0 \mathrm{~mL} / \mathrm{mmol}$ aryl halide), consisting of degassed $n$-butanol $(n-\mathrm{BuOH})$ and degassed deionized water in the ratio of 2.5:1. The resulting mixture was stirred at room temperature for $16 \mathrm{~h}$. The crude reaction mixture was then filtered through a plug of silica gel using EtOAc eluent and concentrated in vacuo. Purification by column chromatography over silica gel eluting with ethyl acetate and hexanes provided the desired TIPS-protected phenylpyrrole.

\section{General procedure 2. Removal of silyl protecting group using TBAF.}

To a solution of TIPS-protected phenylpyrrole (1.0 equiv) in THF $(2.0 \mathrm{~mL} / \mathrm{mmol} \mathrm{SM})$ at $0{ }^{\circ} \mathrm{C}$ was added tetrabutylammonium fluoride (TBAF, 1.0 M in THF, 2.0 equiv) dropwise. Allow to stir reaction mixture for $15 \mathrm{~min}$ at same temperature the reaction progress monitored by TLC. The reaction mixture was quenched with saturated ammonium chloride $\left(\mathrm{NH}_{4} \mathrm{Cl}\right)$ solution and the resulting biphasic mixture was transferred to a separatory funnel. The organic and aqueous layers were separated and the aqueous layer was extracted with ethyl acetate. The organic layers were combined and washed with saturated sodium chloride, dried with sodium sulfate and 
concentrated under reduced pressure to afford the crude product. Subsequent purification by column chromatography over deactivated silica gel $\left(5 \% \mathrm{NEt}_{3}\right)$, eluting with ethyl acetate and hexanes afforded the desilylated product

\section{General procedure 3. Pictet-Spengler reaction.}

$1 \%$ solution of TFA in DMSO (1 mL), aldehyde (1 eq) and pyrrole aniline (1 eq) were added at rt. The mixture was further stirred at ambient temperature and the progress of reaction was monitored by TLC. Upon completion of the reaction, saturated aqueous $\mathrm{NaHCO}_{3}$ was added to quench the acid in the reaction mixture. The product was extracted using ethyl acetate $(20 \mathrm{~mL})$ and the organic layer was washed with water $(2 \times 10 \mathrm{~mL})$, brine solution $(1 \times 10 \mathrm{~mL})$ and finally dried over anhydrous sodium sulfate. It was then evaporated in vacuo to obtain a residue and purified by column chromatography eluting with ethyl acetate and hexanes to afford pure pyonitrins.

\section{General 2D ${ }^{1} \mathrm{H}^{15} \mathrm{~N}$ HMBC monitoring of Pictet-Spengler condensation:}

${ }^{15} \mathrm{~N}$-dechloroaminopyrrolnitrin $\mathbf{2 6}$, and aeruginaldehyde 7 was added to $700 \mu \mathrm{L}$ of $1 \%$ TFA in DMSO- $d_{6}$ in a $5 \mathrm{~mm}$ thin wall, 8 inch NMR tube (Wilmad) and inserted Bruker Avance III HD $800 \mathrm{MHz}$ instrument. The ${ }^{1} \mathrm{H}-{ }^{15} \mathrm{~N}$ HMBC experiment was optimized for ${ }^{\mathrm{N}} J_{\mathrm{NH}}=5 \mathrm{~Hz}$. The ${ }^{1} \mathrm{H}-$ ${ }^{15} \mathrm{~N}$ HMBC data were acquired as $2048 \times 172$ points with 8 transients per $\mathrm{t} 1$ increment. A delay of 1 second was used between transients taking 27 minutes and 43 seconds per spectrum. Instrument was tuned, locked, and shimmed. NMR experiments were then queued to run in the following order: ${ }^{1} \mathrm{H}$ NMR experiment (32 scans taking 2 minutes and 15 seconds) followed by ${ }^{1} \mathrm{H}-{ }^{15} \mathrm{~N}$ HMBC ( 8 scans of 86 increments taking 27 minutes and 43 seconds). Therefore, a single ${ }^{1} \mathrm{H}$ NMR spectrum was collected once every 30 min along with one HMBC spectra constantly for 48 hours.

\section{Synthesis of methyl 2-(2-hydroxyphenyl)thiazole-4-carboxylate (12)}

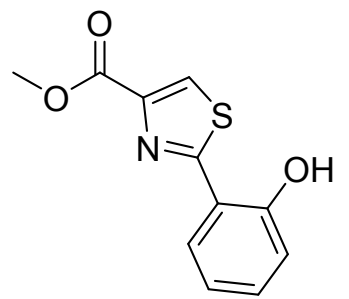

To the anhydrous methanol solution $(50 \mathrm{~mL})$ of the 2-hydroxybenzonitrile $\mathbf{1 0}(2 \mathrm{gm}, 16.8 \mathrm{mmol})$ and anhydrous $\mathrm{Na}_{2} \mathrm{CO}_{3}(1.7 \mathrm{gm}, 16.8 \mathrm{mmol})$ the L-Cysteine methyl ester hydrochloride 9 (7.1 gm $42.01 \mathrm{mmol}$ ) was added, then the resulting mixture was stirred at $80{ }^{\circ} \mathrm{C}$ in oil bath for $12 \mathrm{~h}$, cooled to room temperature. The insoluble solid was filtered and the filtrate was then concentrated. The crude product was dissolved in water $(30 \mathrm{~mL})$ and extracted with ethyl acetate $(2 \times 50 \mathrm{~mL})$. The combined organics were then washed with brine and dried over sodium sulfate and concentrated to obtain pure thiazoline product 11 ( $3 \mathrm{gm}, 77 \%$ yield) was used for next step without further purification. 
Thiazoline 11 (3 gm, $0.012 \mathrm{~mol})$ was dissolved in dry $\mathrm{CH}_{2} \mathrm{Cl}_{2}(150 \mathrm{~mL})$ and cooled to -20 ${ }^{\circ} \mathrm{C}$. DBU $(1.2 \mathrm{~mL}, 0.012 \mathrm{~mol})$ was added and the reaction mixture was stirred for $5 \mathrm{~min}$. at -20 ${ }^{\circ} \mathrm{C}$, treated with $\mathrm{BrCCl}_{3} 3(3.8 \mathrm{~mL}, 0.025 \mathrm{~mol})$ and stirred at room temperature for $12 \mathrm{~h}$. The reaction mixture was then diluted with ammonium chloride $(50 \mathrm{~mL})$ and the aqueous layer was extracted with dichloromehane ( $3 \times 100 \mathrm{~mL}$ each). After drying over sodium sulfate the solvent was removed under reduced pressure, and purified by column chromatography over silica gel, eluting with ethyl acetate and hexanes to furnished $2.8 \mathrm{gm}$ of methyl 2-(2hydroxyphenyl)thiazole-4-carboxylate $\mathbf{1 2}$ (94\% yield) as a colorless solid.

${ }^{1} \mathrm{H}$ NMR $\left(500 \mathrm{MHz}, \mathrm{CDCl}_{3}\right) \delta 11.84(\mathrm{~s}, 1 \mathrm{H}), 8.11(\mathrm{~s}, 1 \mathrm{H}), 7.62(\mathrm{dd}, J=7.8,1.2 \mathrm{~Hz}, 1 \mathrm{H}), 7.36$ (td, $J=7.7,1.2 \mathrm{~Hz}, 1 \mathrm{H}), 7.09$ (d, $J=8.3 \mathrm{~Hz}, 1 \mathrm{H}), 6.93(\mathrm{td}, J=7.7,0.9 \mathrm{~Hz}, 1 \mathrm{H}), 3.97(\mathrm{~s}, 3 \mathrm{H})$.

${ }^{13} \mathrm{C}$ NMR $\left(125 \mathrm{MHz}, \mathrm{CDCl}_{3}\right) \delta 169.7,161.1,157.2,145.9,132.7,127.5,125.3,119.7,118.3$, 116.4, 52.6.

HRMS (ESI) $m / z:[\mathrm{M}+\mathrm{H}]^{+}$Calcd for $\mathrm{C}_{11} \mathrm{H}_{10} \mathrm{NO}_{3} \mathrm{~S}$ 236.0381; Found 236.0372

\section{Synthesis of 2-(2-hydroxyphenyl)-N-methoxy-N-methylthiazole-4-carboxamide (13)}

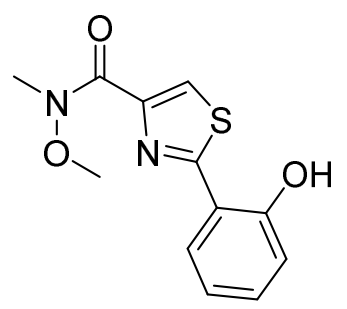

Methyl 2-(2-hydroxyphenyl) thiazole-4-carboxylate 12 (2.8 gm, $0.011 \mathrm{~mol})$ was taken into $50 \mathrm{ml}$ mixture of THF: $\mathrm{H}_{2} \mathrm{O}: \mathrm{CH}_{3} \mathrm{OH}$ (3:2:1). To this lithium hydroxide (1.0 gm, $\left.0.025 \mathrm{~mol}\right)$ was added and the reaction mixture was stirred at room temperature for $2 \mathrm{~h}$. The solvent was removed via rotovap and the reaction mixture was acidified using $1 \mathrm{~N} \mathrm{HCl}$. The white solid precipitate was filtered and dried over vacuum to obtain ( $2.6 \mathrm{gm}, 98 \%$ yield) pure thiazole acid product.

The acid product $(2.5 \mathrm{gm}, 0.011 \mathrm{~mol})$ was then dissolved in dry DMF $(30 \mathrm{~mL})$.To this EDC (2.5 gm, $0.013 \mathrm{~mol}), \mathrm{HOBt}$ (3.35 gm, $0.024 \mathrm{~mol}), N, O$-Dimethylhydroxylamine hydrochloride $(1.31 \mathrm{gm}, 0.013 \mathrm{~mol})$ and DIPEA $(10 \mathrm{~mL}, 0.056 \mathrm{~mol})$ were added at $0{ }^{\circ} \mathrm{C}$ and the reaction mixture was stirred at room temperature for $12 \mathrm{~h}$. The reaction mixture was diluted with water $(50 \mathrm{~mL})$ and extracted with ethyl acetate $(100 \mathrm{~mL} \times 2)$.The combined organic layer was washed with water $(50 \mathrm{~mL})$ and brine $(50 \mathrm{~mL})$ and dried over sodium sulfate and concentrated to obtain pure white solid product 13 ( $2.4 \mathrm{gm}, 80 \%$ yield)

${ }^{1} \mathrm{H}$ NMR $\left(500 \mathrm{MHz}, \mathrm{CDCl}_{3}\right) \delta 11.94(\mathrm{~s}, 1 \mathrm{H}), 8.02(\mathrm{~s}, 1 \mathrm{H}), 7.64(\mathrm{dd}, J=7.8,1.3 \mathrm{~Hz}, 1 \mathrm{H}), 7.35$ $(\operatorname{td}, J=7.9,1.4 \mathrm{~Hz}, 1 \mathrm{H}), 7.07(\mathrm{dd}, J=8.3,0.7 \mathrm{~Hz}, 1 \mathrm{H}), 6.93(\mathrm{td}, J=7.6,1.0 \mathrm{~Hz}, 1 \mathrm{H}), 3.81(\mathrm{~s}$, $3 \mathrm{H}), 3.43(\mathrm{~s}, 3 \mathrm{H})$.

${ }^{13} \mathrm{C}$ NMR $\left(125 \mathrm{MHz}, \mathrm{CDCl}_{3}\right) \delta 168.7,161.7,157.0,147.6,132.5,127.5,123.9,119.7,118.1$, $116.7,62.0,34.0$

HRMS (ESI) $m / z$ : $[\mathrm{M}+\mathrm{H}]^{+}$Calcd for $\mathrm{C}_{12} \mathrm{H}_{13} \mathrm{~N}_{2} \mathrm{O}_{3} \mathrm{~S}$ 265.0646; Found 265.0635 


\section{Synthesis of 2-(2-hydroxyphenyl)thiazole-4-carbaldehyde (7)}<smiles>O=Cc1csc(-c2ccccc2O)n1</smiles>

2-(2-hydroxyphenyl)-N-methoxy-N-methylthiazole-4-carboxamide 13 (100 mg, $0.37 \mathrm{mmol}$ ) was taken in dry THF $(5 \mathrm{~mL})$ and to it $2 \mathrm{M}$ solution of lithium aluminium hydride $(0.284 \mathrm{~mL}, 0.56$ mmol) was added at $0{ }^{\circ} \mathrm{C}$. After $10 \mathrm{~min}$ the reaction was quenched by adding a saturated solution of ammonium chloride $(10 \mathrm{~mL})$ and extracted with ethyl acetate $(30 \mathrm{~mL} \times 2)$. The combined organic layer was washed with water $(30 \mathrm{~mL})$ and brine $(30 \mathrm{~mL})$ and dried over sodium sulfate and concentrated to obtain pure yellow solid product 7 ( $60 \mathrm{mg}, 61 \%$ yield).

${ }^{1} \mathrm{H}$ NMR $\left(500 \mathrm{MHz}, \mathrm{CDCl}_{3}\right) \delta 11.60(\mathrm{~s}, 1 \mathrm{H}), 10.06(\mathrm{~s}, 1 \mathrm{H}), 8.13(\mathrm{~s}, 1 \mathrm{H}), 7.64(\mathrm{dd}, J=7.8,1.2$ $\mathrm{Hz}, 1 \mathrm{H}), 7.39$ (td, $J=7.8,1.2 \mathrm{~Hz}, 1 \mathrm{H}), 7.10(\mathrm{dd}, J=8.3,0.7 \mathrm{~Hz}, 1 \mathrm{H}), 6.95$ (td, $J=7.6,0.8 \mathrm{~Hz}$, $1 \mathrm{H})$.

${ }^{13} \mathrm{C}$ NMR $\left(125 \mathrm{MHz}, \mathrm{CDCl}_{3}\right) \delta 183.5,170.6,157.0,153.7,133.0,127.7,125.2,119.9,118.3$, 116.2 .

HRMS (ESI) $m / z$ : [M + H] $]^{+}$Calcd for $\mathrm{C}_{10} \mathrm{H}_{8} \mathrm{NO}_{2} \mathrm{~S}$ 206.0275; Found 206.0272

\section{2-chloro-6-(1-(triisopropylsilyl)-1H-pyrrol-3-yl)aniline (18) ${ }^{1}$}

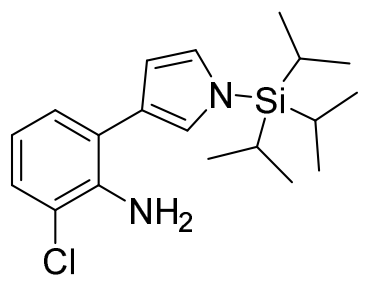

Following general procedure 1, Compound 18 was prepared from 2-chloro-6-iodoaniline 14 (250 $\mathrm{mg}, 0.99 \mathrm{mmol})$ and $17(415 \mathrm{mg}, 1.19 \mathrm{mmol})$. The crude residue was purified by column chromatography over silica gel (hexane/ethyl acetate =9/1), affording $18264 \mathrm{mg}$ (75\% yield) as colorless oil.

${ }^{1} \mathrm{H}$ NMR $\left(500 \mathrm{MHz}, \mathrm{CDCl}_{3}\right) \delta 7.17(\mathrm{~d}, J=7.8 \mathrm{~Hz}, 2 \mathrm{H}), 6.99(\mathrm{t}, J=1.9 \mathrm{~Hz}, 1 \mathrm{H}), 6.88(\mathrm{t}, J=2.2$ $\mathrm{Hz}, 1 \mathrm{H}), 6.71(\mathrm{t}, J=7.8 \mathrm{~Hz}, 1 \mathrm{H}), 6.52(\mathrm{dd}, J=1.5,2.4 \mathrm{~Hz}, 1 \mathrm{H}), 4.39$ (s, 2H), 1.50 (sept, $J=7.4$ $\mathrm{Hz}, 3 \mathrm{H}), 1.15$ (d, $J=7.6 \mathrm{~Hz}, 18 \mathrm{H})$.

${ }^{13} \mathrm{C}$ NMR $\left(125 \mathrm{MHz}, \mathrm{CDCl}_{3}\right) \delta 140.6,128.2,127.1,125.1,124.0,123.6,122.7,119.7,118.3$, $110.8,17.9,11.8$.

HRMS (ESI) $m / z$ : $[\mathrm{M}+\mathrm{H}]^{+}$Calcd for $\mathrm{C}_{19} \mathrm{H}_{30} \mathrm{ClN}_{2} \mathrm{Si} 349.1866$; Found 349.1869 
2-chloro-6-(1H-pyrrol-3-yl)aniline (22) ${ }^{1}$<smiles>Nc1c(Cl)cccc1-c1cc[nH]c1</smiles>

Following general procedure 2, 22 was prepared from 2-chloro-6-(1-(triisopropylsilyl)-1Hpyrrol-3-yl)aniline 18 (50 $\mathrm{mg}, 0.14 \mathrm{mmol}$ ). The crude residue was purified by column chromatography over deactivated silica gel $\left(5 \% \mathrm{NEt}_{3}\right)$ (hexane/ethyl acetate $\left.=2 / 8\right)$, affording compound $22(21 \mathrm{mg})$ as colorless oil in $76 \%$ yield.

${ }^{1} \mathrm{H}$ NMR $\left(500 \mathrm{MHz}, \mathrm{CDCl}_{3}\right) \delta 8.43(\mathrm{~s}, 1 \mathrm{H}), 7.19(\mathrm{~d}, J=8.0 \mathrm{~Hz}, 1 \mathrm{H}), 7.15(\mathrm{~d}, J=7.5 \mathrm{~Hz}, 1 \mathrm{H})$, $6.99(\mathrm{~d}, J=1.3 \mathrm{~Hz}, 1 \mathrm{H}), 6.88(\mathrm{~d}, J=1.9 \mathrm{~Hz}, 1 \mathrm{H}), 6.71$ (t, $J=7.8 \mathrm{~Hz}, 1 \mathrm{H}), 6.45$ (d, $J=1.2 \mathrm{~Hz}$, $1 \mathrm{H}), 4.39$ (s, 2H).

${ }^{13} \mathrm{C}$ NMR $\left(125 \mathrm{MHz}, \mathrm{CDCl}_{3}\right) \delta 140.7,128.4,127.3,123.7,121.5,119.7,118.8,118.4,116.5$, 108.7.

HRMS (ESI) $m / z:[\mathrm{M}+\mathrm{H}]^{+}$Calcd for $\mathrm{C}_{10} \mathrm{H}_{10} \mathrm{ClN}_{2}$ 193.0532; Found 193.0531

\section{pyonitrin A (1)}

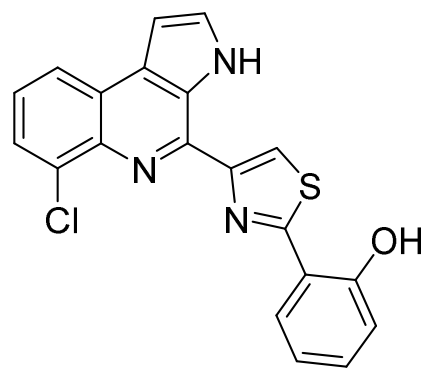

Following general procedure 3, pyonitrin A was prepared from 2-chloro-6-(1H-pyrrol-3yl)aniline $22(2 \mathrm{mg}, 0.01 \mathrm{mmol})$ and $7(2.1 \mathrm{mg}, 0.01 \mathrm{mmol})$. The crude residue was purified by column chromatography over silica gel (hexane/ethyl acetate $=4 / 6)$, affording pyonitrin A $(2.4$ $\mathrm{mg}$ ) as a yellow solid in $61 \%$ yield.

${ }^{1} \mathrm{H}$ NMR (500 MHz, DMSO-d 6 ) $\delta 11.68(\mathrm{~s}, 1 \mathrm{H}), 11.18(\mathrm{~s}, 1 \mathrm{H}), 8.75(\mathrm{dd}, J=7.8,1.2 \mathrm{~Hz}, 1 \mathrm{H})$, $8.70(\mathrm{~s}, 1 \mathrm{H}), 8.34(\mathrm{dd}, J=8.1,1.1 \mathrm{~Hz}, 1 \mathrm{H}), 7.85$ (t, $J=2.7 \mathrm{~Hz}, 1 \mathrm{H}), 7.77(\mathrm{dd}, J=7.4,1.0 \mathrm{~Hz}$, $1 \mathrm{H}), 7.55(\mathrm{t}, J=7.8 \mathrm{~Hz}, 1 \mathrm{H}), 7.39(\mathrm{~m}, 1 \mathrm{H}), 7.31(\mathrm{t}, J=2.0 \mathrm{~Hz}, 1 \mathrm{H}), 7.10(\mathrm{t}, J=8.1 \mathrm{~Hz}, 1 \mathrm{H}), 7.08$ $(\mathrm{t}, J=7.2 \mathrm{~Hz}, 1 \mathrm{H})$

${ }^{13} \mathrm{C}$ NMR $\left(125 \mathrm{MHz}, \mathrm{DMSO}-d_{6}\right) \delta 163.5,155.1,152.9,139.3,137.7,132.4,131.2,129.8,129.6$, 128.5, 126.2, 125.6, 124.7, 122.5, 121.4, 119.5, 119.4, 116.2, 101.3.

HRMS (ESI) $m / z$ : $[\mathrm{M}+\mathrm{H}]^{+}$Calcd for $\mathrm{C}_{20} \mathrm{H}_{13} \mathrm{ClN}_{3} \mathrm{OS} 378.0467$; Found 378.0460 
Table S1: Comparisons of NMR data of pyonitrin $A$ isolated and synthetic sample in DMSO-d 6

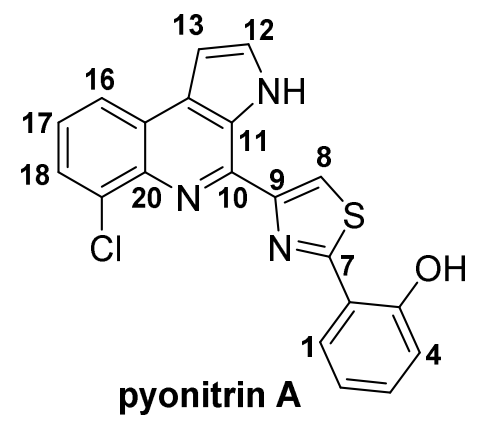

\begin{tabular}{|c|c|c|c|c|c|c|}
\hline No & $\begin{array}{c}{ }^{1} \mathrm{H} \text { NMR } \\
\text { natural (600 } \\
\mathrm{MHz})\end{array}$ & $\begin{array}{c}{ }^{1} \mathrm{H} \text { NMR synthetic } \\
(500 \mathrm{MHz})\end{array}$ & $\begin{array}{l}\text { Difference } \\
\text { in ppm }\end{array}$ & $\begin{array}{c}{ }^{13} \mathrm{C} \mathrm{NMR} \\
\text { natural } \\
(600 \mathrm{MHz})\end{array}$ & $\begin{array}{c}{ }^{13} \mathrm{C} \mathrm{NMR} \\
\text { synthetic } \\
(500 \mathrm{MHz})\end{array}$ & $\begin{array}{l}\text { Difference } \\
\text { in ppm }\end{array}$ \\
\hline 1 & $8.70(\mathrm{~d}, 7.8)$ & $\begin{array}{c}8.75(\mathrm{dd}, J=7.8,1.2 \\
\mathrm{Hz}, 1 \mathrm{H})\end{array}$ & 0.05 & $128.7 \mathrm{CH}$ & 128.5 & 0.2 \\
\hline 2 & $7.00(t, 7.5)$ & $7.10(\mathrm{t}, J=8.1 \mathrm{~Hz}, 1 \mathrm{H})$ & 0.10 & $118.9 \mathrm{CH}$ & 119.5 & 0.6 \\
\hline 3 & $7.34(\mathrm{t}, 7.6)$ & $7.39(\mathrm{~m}, 1 \mathrm{H})$ & 0.05 & $131.6 \mathrm{CH}$ & 131.1 & 0.5 \\
\hline 4 & $7.07(\mathrm{t}, 8.2)$ & $7.08(\mathrm{t}, J=7.2 \mathrm{~Hz}, 1 \mathrm{H})$ & 0.01 & $117.1 \mathrm{CH}$ & 116.2 & 0.9 \\
\hline 5 & & & & $157.3 \mathrm{C}$ & 155.1 & 2.2 \\
\hline 6 & & & & $119.9 \mathrm{C}$ & 119.4 & 0.5 \\
\hline 7 & & & & $164.4 \mathrm{C}$ & 163.5 & 0.9 \\
\hline 8 & $8.64(\mathrm{~s})$ & $8.70(\mathrm{~s}, 1 \mathrm{H})$ & 0.06 & $121.4 \mathrm{C}$ & 121.4 & - \\
\hline 9 & & & & $153.2 \mathrm{C}$ & 152.9 & 0.3 \\
\hline 10 & & & & $139.8 \mathrm{C}$ & 139.2 & 0.6 \\
\hline 11 & & & & $126.7 \mathrm{C}$ & 126.2 & 0.5 \\
\hline 12 & $7.85(\mathrm{~d}, 2.9)$ & $7.85(\mathrm{t}, J=2.7 \mathrm{~Hz}, 1 \mathrm{H})$ & - & $130.1 \mathrm{CH}$ & 129.6 & 0.5 \\
\hline 13 & $7.29(\mathrm{~d}, 2.9)$ & $7.31(\mathrm{t}, J=2.0 \mathrm{~Hz}, 1 \mathrm{H})$ & 0.02 & $101.7 \mathrm{CH}$ & 101.3 & 0.4 \\
\hline 14 & & & & $130.2 \mathrm{C}$ & 129.6 & 0.6 \\
\hline 15 & & & & $125.1 \mathrm{C}$ & 125.5 & 0.4 \\
\hline 16 & $8.32(\mathrm{~d}, 8.0)$ & $\begin{array}{c}8.34(\mathrm{dd}, J=8.1,1.1 \\
\mathrm{Hz}, 1 \mathrm{H})\end{array}$ & 0.02 & $122.9 \mathrm{CH}$ & 122.5 & 0.4 \\
\hline 17 & $7.54(\mathrm{~d}, 7.8)$ & $7.55(\mathrm{t}, J=7.8 \mathrm{~Hz}, 1 \mathrm{H})$ & 0.01 & $126.1 \mathrm{CH}$ & 125.6 & 0.5 \\
\hline 18 & $7.76(\mathrm{~d}, 7.4)$ & $\begin{array}{c}7.77(\mathrm{dd}, J=7.4,1.0 \\
\mathrm{Hz}, 1 \mathrm{H})\end{array}$ & 0.01 & $126.7 \mathrm{CH}$ & 126.2 & 0.5 \\
\hline 19 & & & & 132.8 & 132.4 & 0.4 \\
\hline 20 & & & & 138.2 & 137.7 & 0.5 \\
\hline $\mathrm{NH}$ & 11.69 (bs) & 11.68 & 0.01 & & & \\
\hline $\mathrm{OH}$ & & 11.18 & & & & \\
\hline
\end{tabular}

Synthesis of pyonitrin B:

2-(4-chloro-1-(triisopropylsilyl)-1H-pyrrol-3-yl)aniline (19) ${ }^{1}$ 


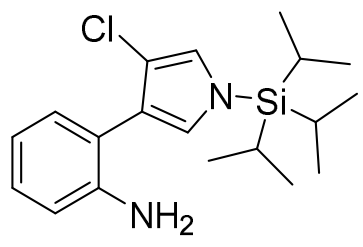

Following general procedure 1, 19 was prepared from 2-iodoaniline 15 (180mg, $0.82 \mathrm{mmol})$ and $16(377 \mathrm{mg}, 0.98 \mathrm{mmol})$. The crude residue was purified by column chromatography over silica gel (hexane/ethyl acetate $=9 / 1$ ), affording $19(192 \mathrm{mg}$ ) as colorless oil in $67 \%$ yield.

${ }^{1} \mathrm{H}$ NMR $\left(500 \mathrm{MHz}, \mathrm{CDCl}_{3}\right) \delta 7.21(\mathrm{~d}, J=7.5 \mathrm{~Hz}, 1 \mathrm{H}), 7.14(\mathrm{t}, J=7.2 \mathrm{~Hz}, 1 \mathrm{H}), 6.79(\mathrm{~m}, 4 \mathrm{H})$, 3.81 (s, 2H), 1.45 (Sept, $J=7.4 \mathrm{~Hz}, 3 \mathrm{H}), 1.13$ (d, $J=7.5 \mathrm{~Hz}, 18 \mathrm{H})$.

${ }^{13} \mathrm{C}$ NMR $\left(125 \mathrm{MHz}, \mathrm{CDCl}_{3}\right) \delta 144.9,131.6,128.4,123.7,121.8,121.7,119.5,118.3,115.5$, 113.8, 17.9, 11.7 .

HRMS (ESI) $m / z:[\mathrm{M}+\mathrm{H}]^{+}$Calcd for $\mathrm{C}_{19} \mathrm{H}_{30} \mathrm{ClN}_{2} \mathrm{Si} 349.1866$; Found 349.1868

\section{2-(4-chloro-1H-pyrrol-3-yl)aniline (23) ${ }^{1}$}<smiles>Nc1ccccc1-c1c[nH]cc1Cl</smiles>

Following general procedure 2, 23 was prepared from 2-(4-chloro-1-(triisopropylsilyl)-1Hpyrrol-3-yl)aniline 19 (60 $\mathrm{mg}, 0.17 \mathrm{mmol})$. The crude residue was purified by column chromatography over deactivated silica gel $\left(5 \% \mathrm{NEt}_{3}\right)$ (hexane/ethyl acetate $\left.=2 / 8\right)$, affording 23 $(28 \mathrm{mg}$ ) as colorless oil in $84 \%$ yield.

${ }^{1} \mathrm{H}$ NMR $\left(500 \mathrm{MHz}, \mathrm{CDCl}_{3}\right) \delta 8.39(\mathrm{~s}, 1 \mathrm{H}), 7.20(\mathrm{dd}, J=7.4,1.0 \mathrm{~Hz}, 1 \mathrm{H}), 7.16(\mathrm{td}, J=7.9,1.3$ $\mathrm{Hz}, 1 \mathrm{H}), 6.83-6.75(\mathrm{~m}, 4 \mathrm{H}), 3.83(\mathrm{~s}, 2 \mathrm{H})$.

${ }^{13} \mathrm{C}$ NMR $\left(125 \mathrm{MHz}, \mathrm{CDCl}_{3}\right) \delta 144.9,131.8,128.6,119.7,119.3,118.4,117.2,116.1,115.5$, 112.1.

HRMS (ESI) $m / z:[\mathrm{M}+\mathrm{H}]^{+}$Calcd for $\mathrm{C}_{10} \mathrm{H}_{10} \mathrm{ClN}_{2}$ 193.0532; Found 193.0530

pyonitrin B (2)<smiles>Oc1ccccc1-c1nc(-c2nc3ccccc3c3c(Cl)c[nH]c23)cs1</smiles> 
Following general procedure 3, 2 was prepared from 2-(4-chloro-1H-pyrrol-3-yl)aniline $\mathbf{2 3}$ (14 $\mathrm{mg}, 0.07 \mathrm{mmol})$ and $7(15 \mathrm{mg}, 0.07 \mathrm{mmol})$. The crude residue was purified by column chromatography over silica gel (hexane/ethyl acetate $=4 / 6$ ), affording pyonitrin B $(15 \mathrm{mg})$ as a colorless oil in $55 \%$ yield.

${ }^{1} \mathrm{H}$ NMR $\left(800 \mathrm{MHz}\right.$, DMSO- $\left.d_{6}\right) \delta 11.83(\mathrm{~s}, 1 \mathrm{H}), 11.20(\mathrm{~s}, 1 \mathrm{H}), 8.86(\mathrm{dd}, J=7.5,1.4 \mathrm{~Hz}, 1 \mathrm{H})$, $8.71(\mathrm{~s}, 1 \mathrm{H}), 8.70(\mathrm{~d}, J=6.8,1 \mathrm{H}), 8.15(\mathrm{dd}, J=7.6,1.2 \mathrm{~Hz}, 1 \mathrm{H}), 7.89(\mathrm{~d}, J=2.8 \mathrm{~Hz}, 1 \mathrm{H}), 7.70-$ $7.67(\mathrm{~m}, 2 \mathrm{H}), 7.40(\mathrm{dt}, J=7.5,1.4 \mathrm{~Hz}, 1 \mathrm{H}), 7.11(\mathrm{~d}, J=8.1 \mathrm{~Hz}, 1 \mathrm{H}), 7.09(\mathrm{t}, J=7.4 \mathrm{~Hz}, 1 \mathrm{H})$.

${ }^{13} \mathrm{C}$ NMR $\left(200 \mathrm{MHz}\right.$, DMSO- $\left.d_{6}\right) \delta 163.5,155.1,152.5,142.1,139.3,131.2,129.4,128.5,126.5$, 126.2, 126.0, 125.2, 123.1, 122.3, 121.8, 121.2, 119.5, 119.4, 116.2, 105.2.

HRMS (ESI) $m / z$ : $[\mathrm{M}+\mathrm{H}]^{+}$Calcd for $\mathrm{C}_{20} \mathrm{H}_{13} \mathrm{ClN}_{3} \mathrm{OS}$ 378.0467; Found 378.0464

Table S2: Comparisons of NMR data of pyonitrin B isolated and synthetic sample in DMSO-d 6

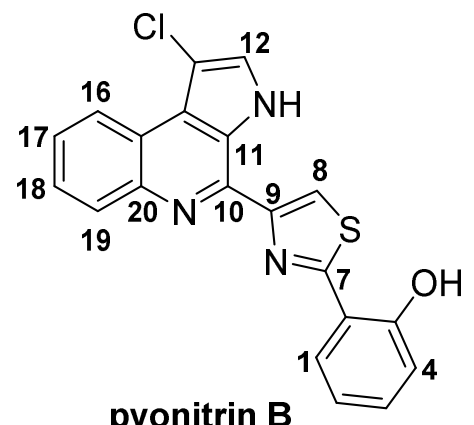

\begin{tabular}{|c|c|c|c|c|c|c|}
\hline No & $\begin{array}{c}{ }^{1} \mathrm{H} \text { NMR } \\
\text { natural }(600 \\
\text { MHz) }\end{array}$ & $\begin{array}{c}{ }^{1} \mathrm{H} \text { NMR synthetic } \\
(500 \mathrm{MHz})\end{array}$ & $\begin{array}{c}\text { Difference } \\
\text { in ppm }\end{array}$ & $\begin{array}{c}{ }^{13} \mathrm{C} \text { NMR } \\
\text { natural } \\
(600 \mathrm{MHz})\end{array}$ & $\begin{array}{c}{ }^{13} \mathrm{C} \text { NMR } \\
\text { synthetic } \\
(500 \mathrm{MHz})\end{array}$ & $\begin{array}{c}\text { Difference } \\
\text { in ppm }\end{array}$ \\
\hline 1 & $8.64(\mathrm{~d}, 7.3)$ & $8.70(\mathrm{dd}, J=6.8,1 \mathrm{H})$ & 0.06 & 128.6 & 128.5 & 0.1 \\
\hline 2 & $7.09(\mathrm{t}, 7.7)$ & $\begin{array}{c}7.11(\mathrm{~d}, J=8.1 \mathrm{~Hz}, \\
1 \mathrm{H}),\end{array}$ & 0.02 & 117.6 & 116.2 & 1.4 \\
\hline 3 & $7.30(\mathrm{t}, 7.3)$ & $\begin{array}{c}7.40(\mathrm{dt}, J=7.5,1.4 \\
\mathrm{Hz}, 1 \mathrm{H})\end{array}$ & 0.10 & 131.5 & 131.2 & 0.3 \\
\hline 4 & $6.93(\mathrm{t}, 7.1)$ & $\begin{array}{c}7.09(\mathrm{t}, J=7.4 \mathrm{~Hz}, \\
1 \mathrm{H})\end{array}$ & 0.16 & 118.1 & 119.4 & 1.3 \\
\hline 5 & & & & 158.0 & 155.05 & 2.95 \\
\hline 6 & & & & 119.8 & 119.5 & 0.3 \\
\hline 7 & & & & 164.6 & 163.5 & 1.1 \\
\hline 8 & $8.62(\mathrm{~s})$ & $8.71(\mathrm{~s}, 1 \mathrm{H})$, & 0.09 & 121.1 & 121.2 & 0.1 \\
\hline 9 & & & & 152.7 & 152.5 & 0.2 \\
\hline 10 & & & -140.0 & 139.3 & 0.7 \\
\hline 11 & & & 123.4 & 121.8 & 1.6 \\
\hline 12 & $7.88(\mathrm{~s})$ & $7.88(\mathrm{~d}, J=2.8 \mathrm{~Hz}$, & $-1 \mathrm{H})$ & & 126.5 & 0.3 \\
\hline 13 & & & & 105.6 & 105.2 & 0.4 \\
\hline
\end{tabular}




\begin{tabular}{|c|c|c|c|c|c|c|}
\hline 14 & & & & 125.8 & 125.2 & 0.6 \\
\hline 15 & & & & 123.0 & 123.1 & 0.1 \\
\hline 16 & $8.85(\mathrm{~d}, 7.9)$ & $\begin{array}{c}8.86(\mathrm{dd}, J=7.5,1.4 \\
\mathrm{Hz}, 1 \mathrm{H})\end{array}$ & 0.01 & 122.3 & 122.3 & -- \\
\hline 17 & $7.65(\mathrm{~m})$ & $7.70-7.67(\mathrm{~m}, 2 \mathrm{H})$ & - & 126.4 & 126.0 & 0.4 \\
\hline 18 & $7.68(\mathrm{~m})$ & & - & 126.9 & 126.2 & 0.7 \\
\hline 19 & $8.14(\mathrm{~d}, 7.7)$ & $\begin{array}{c}8.16(\mathrm{dd}, J=7.6,1.2 \\
\mathrm{Hz}, 1 \mathrm{H})\end{array}$ & 0.02 & 129.9 & 129.4 & 0.3 \\
\hline 20 & & & & 142.7 & 142.1 & 0.6 \\
\hline $\mathrm{NH}$ & & 11.83 & & & & \\
\hline $\mathrm{OH}$ & & 11.20 & & & & \\
\hline
\end{tabular}

Synthesis of pyonitrin C:

2-(1-(triisopropylsilyl)-1H-pyrrol-3-yl)aniline (20) ${ }^{1}$<smiles>CC(C)[Si](C(C)C)(C(C)C)n1ccc(-c2ccccc2N)c1</smiles>

Following general procedure 1, 20 was prepared from 2-iodoaniline 15 (150 mg, $0.68 \mathrm{mmol})$ and $17(286 \mathrm{mg}, 0.82 \mathrm{mmol})$. The crude residue was purified by column chromatography over silica gel (hexane/ethyl acetate $=9 / 1)$, affording $\mathbf{2 0}(180 \mathrm{mg}$ ) as a colorless oil in $83 \%$ yield.

${ }^{1} \mathrm{H}$ NMR $\left(500 \mathrm{MHz}, \mathrm{CDCl}_{3}\right) \delta 7.26(\mathrm{dd}, J=7.4,1.2 \mathrm{~Hz}, 1 \mathrm{H}), 7.05(\mathrm{td}, J=7.8,1.4 \mathrm{~Hz}, 1 \mathrm{H}), 6.96$ (s, 1H), 6.85 (s, 1H), 6.78 (td, $J=7.5,1.0 \mathrm{~Hz}, 1 \mathrm{H}), 6.75$ (dd, $J=7.8,0.8 \mathrm{~Hz}, 1 \mathrm{H}), 6.51$ (dd, $J=$ 2.4, $1.2 \mathrm{~Hz}, 1 \mathrm{H}), 3.94$ (s, 2H), 1.48 (sept, $J=7.6 \mathrm{~Hz}, 3 \mathrm{H}), 1.13$ (d, $J=7.5 \mathrm{~Hz}, 18 \mathrm{H})$.

${ }^{13} \mathrm{C}$ NMR $\left(125 \mathrm{MHz}, \mathrm{CDCl}_{3}\right) \delta 143.8,129.9,127.1,124.9,124.0,122.8,122.5,118.7,115.6$, 110.9, 18.0, 11.9.

HRMS (ESI) $m / z$ : [M $+\mathrm{H}]^{+}$Calcd for $\mathrm{C}_{19} \mathrm{H}_{31} \mathrm{~N}_{2} \mathrm{Si} 315.2256$; Found 315.2274

\section{2-(1H-pyrrol-3-yl)aniline (24) ${ }^{1}$}<smiles>Nc1ccccc1-c1cc[nH]c1</smiles>

Following general procedure 2, 24 was prepared from 2-(1-(triisopropylsilyl)-1H-pyrrol-3-yl) aniline $20(50 \mathrm{mg}, 0.15 \mathrm{mmol})$. The crude residue was purified by column chromatography over deactivated silica gel $\left(5 \% \mathrm{NEt}_{3}\right)$ (hexane/ethyl acetate $\left.=2 / 8\right)$, affording $24(22 \mathrm{mg})$ as a colorless oil in $87 \%$ yield. 
${ }^{1} \mathrm{H}$ NMR $\left(500 \mathrm{MHz}, \mathrm{CDCl}_{3}\right) \delta 8.39(\mathrm{~s}, 1 \mathrm{H}), 7.26(\mathrm{dd}, J=7.4,1.2 \mathrm{~Hz}, 1 \mathrm{H}), 7.09(\mathrm{td}, J=7.7,1.2$ $\mathrm{Hz}, 1 \mathrm{H}), 7.01(\mathrm{~s}, 1 \mathrm{H}), 6.88(\mathrm{~m}, 1 \mathrm{H}), 6.82-6.77(\mathrm{~m}, 2 \mathrm{H}), 6.47(\mathrm{~m}, 1 \mathrm{H}), 3.73(\mathrm{~s}, 2 \mathrm{H})$.

${ }^{13} \mathrm{C}$ NMR $\left(125 \mathrm{MHz}, \mathrm{CDCl}_{3}\right) \delta 143.9,130.0,127.3,122.4,122.1,118.8,118.6,116.3,115.7$, 108.9 .

HRMS (ESI) $m / z$ : $[\mathrm{M}+\mathrm{H}]^{+}$Calcd for $\mathrm{C}_{10} \mathrm{H}_{11} \mathrm{~N}_{2}$ 159.0922; Found 159.0917

\section{pyonitrin $\mathbf{C}$}

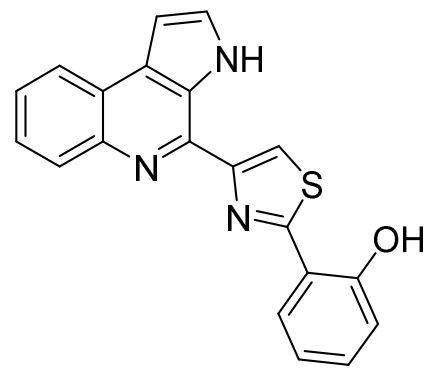

Following general procedure 3, pyonitrin $\mathrm{C}$ was prepared from 2-(1H-pyrrol-3-yl)aniline 24 (10 $\mathrm{mg}, 0.06 \mathrm{mmol})$ and $7(13 \mathrm{mg}, 0.06 \mathrm{mmol})$. The crude residue was purified by column chromatography over silica gel (hexane/ethyl acetate $=4 / 6)$, affording pyonitrin C $(12 \mathrm{mg})$ as a yellow solid in $55 \%$ yield.

${ }^{1} \mathrm{H}$ NMR $\left(800 \mathrm{MHz}, \mathrm{DMSO}-d_{6}\right) \delta 11.59(\mathrm{~s}, 1 \mathrm{H}), 8.72(\mathrm{dd}, J=7.8,1.6 \mathrm{~Hz}, 1 \mathrm{H}), 8.65(\mathrm{~s}, 1 \mathrm{H}), 8.33$ (dd, $J=7.8,1.3 \mathrm{~Hz}, 1 \mathrm{H}), 8.09(\mathrm{~d}, J=7.5 \mathrm{~Hz}, 1 \mathrm{H}), 7.79(\mathrm{t}, J=2.7 \mathrm{~Hz}, 1 \mathrm{H}), 7.62-7.56(\mathrm{~m}, 2 \mathrm{H})$, $7.37(\mathrm{t}, J=7.3 \mathrm{~Hz}, 1 \mathrm{H}), 7.25(\mathrm{~s}, 1 \mathrm{H}), 7.11(\mathrm{~d}, J=8.1 \mathrm{~Hz}, 1 \mathrm{H}), 7.05(\mathrm{~d}, J=7.4 \mathrm{~Hz}, 1 \mathrm{H})$.

${ }^{13} \mathrm{C}$ NMR $\left(200 \mathrm{MHz}\right.$, DMSO- $\left.d_{6}\right) \delta 163.4,153.1,141.9,139.2,131.0,129.3,129.0,128.6,128.4$, 126.0, 125.9, 125.5, 123.2, 123.1, 120.3, 119.5, 119.1, 116.4, 100.8.

HRMS (ESI) $m / z$ : $[\mathrm{M}+\mathrm{H}]^{+}$Calcd for $\mathrm{C}_{20} \mathrm{H}_{14} \mathrm{~N}_{3} \mathrm{OS} 344.0857$; Found 344.0844

\section{Synthesis of pyonitrin D:}

\section{2-chloro-6-(4-chloro-1-(triisopropylsilyl)-1H-pyrrol-3-yl)aniline (21) ${ }^{1}$}<smiles>CC(C)[Si](C(C)C)(C(C)C)n1cc(Cl)c(-c2cccc(Cl)c2N)c1</smiles>

Following general procedure 1, 21 was prepared from 2-chloro-6-iodoaniline 14 (190 mg, 0.75 $\mathrm{mmol}$ ) and 16 ( $345 \mathrm{mg}, 0.90 \mathrm{mmol}$ ). The crude residue was purified by column chromatography over silica gel (hexane/ethyl acetate $=9 / 1)$, affording $21(211 \mathrm{mg})$ as a colorless oil in $73 \%$ yield. 
${ }^{1} \mathrm{H}$ NMR $\left(500 \mathrm{MHz}, \mathrm{CDCl}_{3}\right) \delta 7.23(\mathrm{dd}, J=8.0,1.2 \mathrm{~Hz}, 1 \mathrm{H}), 7.12(\mathrm{dd}, J=7.6,1.2 \mathrm{~Hz}, 1 \mathrm{H}), 6.85$ $-6.80(\mathrm{~m}, 2 \mathrm{H}), 6.72(\mathrm{t}, J=7.8 \mathrm{~Hz}, 1 \mathrm{H}), 4.23(\mathrm{~s}, 2 \mathrm{H}), 1.45$ (sept, $J=7.6 \mathrm{~Hz}, 3 \mathrm{H}), 1.13$ (d, $J=7.5$ $\mathrm{Hz}, 18 \mathrm{H})$.

${ }^{13} \mathrm{C}$ NMR $\left(125 \mathrm{MHz}, \mathrm{CDCl}_{3}\right) \delta 141.7,130.0,128.4,123.4,122.1,121.1,120.7,119.5,118.0$, 113.7, 17.9, 11.7.

HRMS (ESI) $m / z$ : $[\mathrm{M}+\mathrm{H}]^{+}$Calcd for $\mathrm{C}_{19} \mathrm{H}_{29} \mathrm{Cl}_{2} \mathrm{~N}_{2} \mathrm{Si} 383.1477$; Found 383.1479

\section{2-chloro-6-(4-chloro-1H-pyrrol-3-yl)aniline (25) ${ }^{1}$}<smiles>Nc1c(Cl)cccc1-c1c[nH]cc1Cl</smiles>

Following general procedure 2, 25 was prepared from 2-chloro-6-(4-chloro-1-(triisopropylsilyl)1H-pyrrol-3-yl) aniline 21 (100 $\mathrm{mg}, 0.26 \mathrm{mmol})$. The crude residue was purified by column chromatography over deactivated silica gel $\left(5 \% \mathrm{NEt}_{3}\right)$ (hexane/ethyl acetate $\left.=2 / 8\right)$, affording $\mathbf{2 5}$ $(48 \mathrm{mg})$ as a colorless oil in $81 \%$ yield.

${ }^{1} \mathrm{H}$ NMR $\left(500 \mathrm{MHz}, \mathrm{CDCl}_{3}\right) \delta 8.43(\mathrm{~s}, 1 \mathrm{H}), 7.24(\mathrm{dd}, J=8.0,1.3 \mathrm{~Hz}, 1 \mathrm{H}), 7.09$ (dd, $J=7.6,1.2$ $\mathrm{Hz}, 1 \mathrm{H}), 6.86(\mathrm{t}, J=2.6 \mathrm{~Hz}, 1 \mathrm{H}), 6.82(\mathrm{t}, J=2.7 \mathrm{~Hz}, 1 \mathrm{H}), 6.72(\mathrm{t}, J=7.8 \mathrm{~Hz}, 1 \mathrm{H}), 4.23(\mathrm{~s}, 2 \mathrm{H})$.

${ }^{13} \mathrm{C}$ NMR $\left(125 \mathrm{MHz}, \mathrm{CDCl}_{3}\right) \delta 141.8,130.2,128.6,120.3,119.5,119.3,118.0,117.3,116.3$, 112.2 .

HRMS (ESI) $m / z:[\mathrm{M}+\mathrm{H}]^{+}$Calcd for $\mathrm{C}_{10} \mathrm{H}_{9} \mathrm{Cl}_{2} \mathrm{~N}_{2}$ 227.0142; Found 227.0137

\section{pyonitrin D (4)}

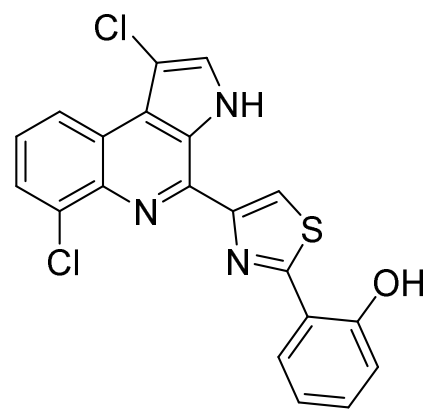

Following general procedure 2, pyonitrin D was prepared from 2-chloro-6-(4-chloro-1H-pyrrol3-yl) aniline 25 (10 mg, $0.04 \mathrm{mmol})$ and $7(9 \mathrm{mg}, 0.04 \mathrm{mmol})$. The crude residue was purified by column chromatography over silica gel (hexane/ethyl acetate $=4 / 6)$, affording pyonitrin D (12 $\mathrm{mg}$ ) as a yellow solid in $66 \%$ yield. 
${ }^{1} \mathrm{H}$ NMR (500 MHz, DMSO-d 6 ) $\delta 11.91(\mathrm{~s}, 1 \mathrm{H}), 11.28(\mathrm{~s}, 1 \mathrm{H}), 8.84(\mathrm{dd}, J=8.3,1.0 \mathrm{~Hz}, 1 \mathrm{H})$, $8.73(\mathrm{~s}, 1 \mathrm{H}), 8.72(\mathrm{~m}, 1 \mathrm{H}), 7.95(\mathrm{~s}, 1 \mathrm{H}), 7.86(\mathrm{dd}, J=7.5,1.0 \mathrm{~Hz}, 1 \mathrm{H}), 7.64(\mathrm{t}, J=7.9 \mathrm{~Hz}, 1 \mathrm{H})$, $7.39(\mathrm{dt}, J=8.1,1.0 \mathrm{~Hz}, 1 \mathrm{H}), 7.11(\mathrm{~d}, J=8.3 \mathrm{~Hz}, 1 \mathrm{H}), 7.08(\mathrm{t}, J=7.5 \mathrm{~Hz}, 1 \mathrm{H})$.

${ }^{13} \mathrm{C}$ NMR $\left(125 \mathrm{MHz}, \mathrm{DMSO}-d_{6}\right) \delta 163.8,152.3,139.4,138.0,132.7,131.2,128.5,127.2,126.9$, $126.2,125.4,123.8,123.4,122.1,121.0,119.4,116.3,105.5$.

HRMS (ESI) $m / z$ : $[\mathrm{M}+\mathrm{H}]^{+}$Calcd for $\mathrm{C}_{20} \mathrm{H}_{12} \mathrm{Cl}_{2} \mathrm{~N}_{3} \mathrm{OS} 412.0078$; Found 412.0075

Synthesis of ${ }^{15} \mathrm{~N}$ labeled pyonitrin A:

${ }^{15}$ N labeled 2-chloro-6-iodoaniline (26a)<smiles>Nc1c(Cl)cccc1I</smiles>

A solution of 2-chloro-6-iodobenzoic acid (281 mg $1.0 \mathrm{mmol})$ in concentrated sulfuric acid (2.5 $\mathrm{mL}$ ) was heated to $60^{\circ} \mathrm{C}$ in oil bath for $1 \mathrm{~h}$. The solution was then cooled to rt before addition of sodium azide $\left[{ }^{15} \mathrm{~N}\right](66 \mathrm{mg}, 1.0 \mathrm{mmol})$. The resulting mixture was left to stir at $\mathrm{rt}$ for $42 \mathrm{~h}$ before cooling to $0^{\circ} \mathrm{C}$ and basifying with concentrated ammonium hydroxide. The organics were extracted with ethyl acetate $(2 \times 20 \mathrm{~mL})$. The organic layers were combined and washed with brine solution ( 1 x $20 \mathrm{~mL}$ ), dried over sodium sulfate and concentrated under reduced pressure and purified by column chromatography over silica gel (hexane/ethyl acetate $=7 / 3$ ), to afford the pure ${ }^{15} \mathrm{~N}$ labeled 2-chloro-6-iodoaniline 26a as brown solid $(210 \mathrm{mg})$ in $82 \%$ yield.

${ }^{1} \mathrm{H}$ NMR $\left(500 \mathrm{MHz}, \mathrm{CDCl}_{3}\right) \delta 7.54(\mathrm{~d}, J=7.9 \mathrm{~Hz}, 1 \mathrm{H}), 7.23(\mathrm{~d}, J=7.9 \mathrm{~Hz}, 1 \mathrm{H}), 6.41(\mathrm{t}, J=7.9$ $\mathrm{Hz}, 1 \mathrm{H}), 4.64-4.41(\mathrm{~m}, 2 \mathrm{H})$.

${ }^{13} \mathrm{C}$ NMR $\left(125 \mathrm{MHz}, \mathrm{CDCl}_{3}\right) \delta 143.5,137.6,129.7,120.0,118.0,83.6$.

HRMS (ESI) $m / z$ : $[\mathrm{M}+\mathrm{H}]^{+}$Calcd for $\mathrm{C}_{6} \mathrm{H}_{6} \mathrm{ClI}^{15} \mathrm{~N} 254.9233$; Found 254.9203

Note: We have observed multiplate for $\mathrm{NH}_{2}$ in proton because equal possibility of the nucleophilic attack of ${ }^{15} \mathrm{~N}$ labeled as well as ${ }^{14} \mathrm{~N}$ nitrogen from the sodium azide $\left(\mathrm{Na}^{15} \mathrm{NN}_{2}\right)$ in the reaction. The same was confirmed by high resolution mass spectroscopy showed the mass for

both ${ }^{14} \mathrm{~N}$ and ${ }^{15} \mathrm{~N}$ products. HRMS (ESI) $\mathrm{m} / z$ : $[\mathrm{M}+\mathrm{H}]^{+}$Calcd for $\mathrm{C}_{6} \mathrm{H}_{6} \mathrm{ClIN}-253.9233$; Found 253.9232

${ }^{15}$ N labeled 2-chloro-6-(1-(triisopropylsilyl)-1H-pyrrol-3-yl)aniline (26b)<smiles>CC(C)[Si](C(C)C)(C(C)C)n1ccc(-c2cccc(Cl)c2N)c1</smiles> 
Following general procedure 1, 26b was prepared from ${ }^{15} \mathrm{~N}$ labelled 2-chloro-6-iodoaniline 26a (100 $\mathrm{mg}, 0.39 \mathrm{mmol})$ and $17(164 \mathrm{mg}, 0.47 \mathrm{mmol})$. The crude residue was purified by column chromatography over silica gel (hexane/ethyl acetate $=9 / 1)$, affording $26 \mathbf{b}(111 \mathrm{mg})$ as a colorless oil in $81 \%$ yield.

${ }^{1} \mathrm{H}$ NMR $\left(500 \mathrm{MHz}, \mathrm{CDCl}_{3}\right) \delta$ 7.16-7.14 (m, 2H), $6.96(\mathrm{~s}, 1 \mathrm{H}), 6.86(\mathrm{t}, J=2.2 \mathrm{~Hz}, 1 \mathrm{H}), 6.69(\mathrm{t}, J$ $=7.8 \mathrm{~Hz}, 1 \mathrm{H}), 6.50(\mathrm{dd}, J=2.4,1.2 \mathrm{~Hz} 1 \mathrm{H}), 4.37(\mathrm{~m}, 2 \mathrm{H}), 1.48(\mathrm{sept}, J=7.6 \mathrm{~Hz}, 3 \mathrm{H}), 1.13(\mathrm{~d}, J$ $=7.5 \mathrm{~Hz}, 18 \mathrm{H})$

${ }^{13} \mathrm{C}$ NMR $\left(125 \mathrm{MHz}, \mathrm{CDCl}_{3}\right) \delta 140.6,128.2,127.1,125.1,124.1,123.6,122.7,119.7,118.4$, $110.8,18.0,11.8$.

HRMS (ESI) $m / z$ : $[\mathrm{M}+\mathrm{H}]]^{+}$Calcd for $\mathrm{C}_{19} \mathrm{H}_{30} \mathrm{Cl}^{15} \mathrm{NNSi} 350.1866$; Found $350.1847\left({ }^{15} \mathrm{~N}\right.$ product) HRMS (ESI) $m / z$ : $[\mathrm{M}+\mathrm{H}]^{+}$Calcd for $\mathrm{C}_{19} \mathrm{H}_{30} \mathrm{ClN}_{2} \mathrm{Si} 349.1866$; Found $349.1871\left({ }^{14} \mathrm{~N}\right.$ product)

\section{${ }^{15} \mathrm{~N}$ labeled 2-chloro-6-(1H-pyrrol-3-yl)aniline (26)}<smiles>Nc1c(Cl)cccc1-c1cc[nH]c1</smiles>

Following general procedure 2, 26 was prepared from ${ }^{15} \mathrm{~N}$ labelled 2-chloro-6-(1(triisopropylsilyl)-1H-pyrrol-3-yl)aniline $26 \mathbf{b}(55 \mathrm{mg}, 0.15 \mathrm{mmol})$. The crude residue was purified by column chromatography over deactivated silica gel $\left(5 \% \mathrm{NEt}_{3}\right)$ (hexane/ethyl acetate $=2 / 8)$, affording $26(22 \mathrm{mg})$ as a colorless oil in $73 \%$ yield.

${ }^{1} \mathrm{H}$ NMR $\left(800 \mathrm{MHz}, \mathrm{DMSO}-d_{6}\right) \delta 11.05(\mathrm{~s}, 1 \mathrm{H}), 7.10-7.08(\mathrm{~m}, 2 \mathrm{H}), 7.01$ (q, $\left.J=1.8 \mathrm{~Hz}, 1 \mathrm{H}\right)$, $6.87(\mathrm{q}, J=2.5 \mathrm{~Hz}, 1 \mathrm{H}), 6.60(\mathrm{t}, J=7.7 \mathrm{~Hz}, 1 \mathrm{H}), 6.27(\mathrm{q}, J=2.4 \mathrm{~Hz}, 1 \mathrm{H}), 4.87-4.76(\mathrm{~m}, 2 \mathrm{H})$.

${ }^{13} \mathrm{C}$ NMR $\left(200 \mathrm{MHz}\right.$, DMSO- $\left.d_{6}\right) \delta 140.6,127.8,126.3,123.6,120.1,118.6,118.2,117.3,116.2$, 107.3.

HRMS (ESI) $m / z$ : $[\mathrm{M}+\mathrm{H}]^{+}$Calcd for $\mathrm{C}_{10} \mathrm{H}_{10} \mathrm{Cl}^{15} \mathrm{NN}$ 194.0532; Found $194.0499\left({ }^{15} \mathrm{~N}\right.$ product) HRMS (ESI) $m / z:[\mathrm{M}+\mathrm{H}]^{+}$Calcd for $\mathrm{C}_{10} \mathrm{H}_{10} \mathrm{ClN}_{2}$ 193.0532; Found $193.0428\left({ }^{14} \mathrm{~N}\right.$ product $)$

\section{${ }^{15}$ N labeled pyonitrin A (29)}

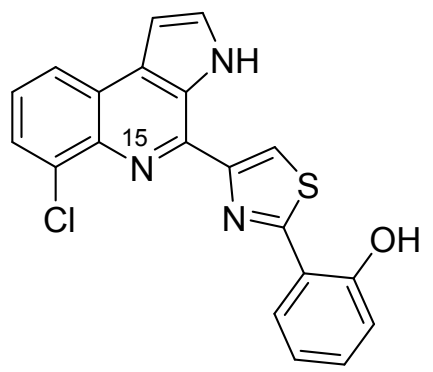

Following general procedure 3, 29 was prepared from ${ }^{15} \mathrm{~N}$ labeled 2-chloro-6-(1H-pyrrol-3yl)aniline 26 (10 $\mathrm{mg}, 0.05 \mathrm{mmol})$ and $7(10.6 \mathrm{mg}, 0.05 \mathrm{mmol})$. The crude residue was purified by 
column chromatography over silica gel (hexane/ethyl acetate $=4 / 6$ ), affording ${ }^{15} \mathrm{~N}$ labeled pyonitrin A (14 mg) as a yellow solid in 71\% yield.

${ }^{1} \mathrm{H}$ NMR (500 MHz, DMSO- $\left.d_{6}\right) \delta 11.69(\mathrm{~s}, 1 \mathrm{H}), 11.18(\mathrm{~s}, 1 \mathrm{H}), 8.75(\mathrm{dd}, J=7.8,1.1 \mathrm{~Hz}, 1 \mathrm{H})$, $8.70(\mathrm{~s}, 1 \mathrm{H}), 8.34(\mathrm{dd}, J=8.1,1.0 \mathrm{~Hz}, 1 \mathrm{H}), 7.85-7.83(\mathrm{~m}, 1 \mathrm{H}), 7.78(\mathrm{~d}, J=7.4 \mathrm{~Hz}, 1 \mathrm{H}), 7.54(\mathrm{t}$, $J=7.8 \mathrm{~Hz}, 1 \mathrm{H}), 7.39(\mathrm{dt}, J=8.2,1.3 \mathrm{~Hz}, 1 \mathrm{H}), 7.31(\mathrm{~s}, 1 \mathrm{H}), 7.11(\mathrm{~d}, J=8.0 \mathrm{~Hz}, 1 \mathrm{H}), 7.09$ (t, $J=$ $7.4 \mathrm{~Hz}, 1 \mathrm{H})$.

${ }^{13} \mathrm{C}$ NMR $\left(125 \mathrm{MHz}, \mathrm{DMSO}-d_{6}\right) \delta 163.5,155.1,152.9,139.3,137.7,132.4,131.2,129.8,129.6$, $128.5,126.2,125.6,124.7,122.5,121.4,119.5,119.4,116.2,101.3$.

HRMS (ESI) $m / z$ : $[\mathrm{M}+\mathrm{H}]^{+}$Calcd for $\mathrm{C}_{20} \mathrm{H}_{13} \mathrm{Cl}^{15} \mathrm{NN}_{2} \mathrm{OS} 379.0467$; Found $379.0438\left({ }^{15} \mathrm{~N}\right.$ product)

HRMS (ESI) $m / z$ : $[\mathrm{M}+\mathrm{H}]^{+}$Calcd for $\mathrm{C}_{20} \mathrm{H}_{13} \mathrm{ClN}_{3} \mathrm{OS} 378.0467$; Found $378.0457\left({ }^{14} \mathrm{~N}\right.$ product)

\section{Synthesis and isolation of ${ }^{15} \mathrm{~N}$ labeled intermediate (28)}

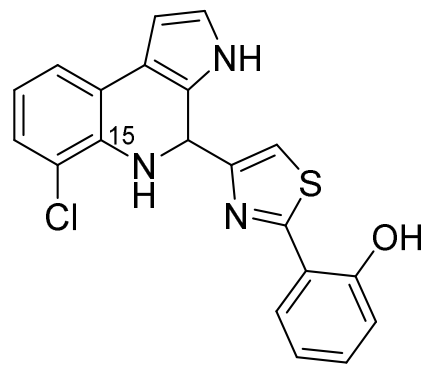

$1 \%$ solution of TFA in DMSO $(1 \mathrm{~mL}),{ }^{15} \mathrm{~N}$ labeled 2-chloro-6-(1H-pyrrol-3-yl)aniline 26 (5 mg, $0.025 \mathrm{mmol})$ and 2-(2-hydroxyphenyl)thiazole-4-carbaldehyde $7(5.31 \mathrm{mg}, 0.025 \mathrm{mmol})$ were added at rt. The mixture was further stirred at ambient temperature for $45 \mathrm{~min}$. Then saturated aqueous $\mathrm{NaHCO}_{3}$ was added to quench the acid in the reaction mixture. The product was extracted using ethyl acetate $(10 \mathrm{~mL} \times 2)$ and the organic layer was washed with water $(10 \mathrm{~mL} x$ $2)$, brine solution $(10 \mathrm{~mL} \times 1)$ and finally dried over anhydrous sodium sulfate. It was then evaporated in vacuo to obtain a residue and purified by flash column chromatography silica gel (hexane/ethyl acetate $=4 / 6)$, to afford pure ${ }^{15} \mathrm{~N}$ labeled intermediate $28(7 \mathrm{mg}, 71 \%$ ).

${ }^{1} \mathrm{H}$ NMR (500 MHz, DMSO- $\left.d_{6}\right) \delta 11.27(\mathrm{~s}, 1 \mathrm{H}), 10.98(\mathrm{~s}, 1 \mathrm{H}), 7.97(\mathrm{dd}, J=7.9,1.5 \mathrm{~Hz}, 1 \mathrm{H})$, $7.36-7.24(\mathrm{~m}, 1 \mathrm{H}), 7.18(\mathrm{~d}, J=6.8 \mathrm{~Hz}, 1 \mathrm{H}), 7.12(\mathrm{~s}, 1 \mathrm{H}), 7.00-6.94(\mathrm{~m}, 2 \mathrm{H}), 6.92(\mathrm{t}, J=7.5$ $\mathrm{Hz}, 1 \mathrm{H}), 6.77(\mathrm{t}, J=2.6 \mathrm{~Hz}, 1 \mathrm{H}), 6.58(\mathrm{t}, J=7.7 \mathrm{~Hz}, 1 \mathrm{H}), 6.35(\mathrm{t}, J=2.4 \mathrm{~Hz}, 1 \mathrm{H}), 6.09(\mathrm{~d}, J=$ $2.5 \mathrm{~Hz}, 1 \mathrm{H})$.

${ }^{13} \mathrm{C}$ NMR $\left(125 \mathrm{MHz}\right.$, DMSO- $\left.d_{6}\right) \delta 165.2,158.1,155.3,136.5,131.2,127.5,125.1,125.0,121.0$, 119.9, 119.4, 119.3, 118.3, 117.4, 116.8, 116.5, 114.2, 114.0, 102.0, 51.3.

HRMS (ESI) $m / z$ : $[\mathrm{M}+\mathrm{H}]^{+}$Calcd for $\mathrm{C}_{20} \mathrm{H}_{15} \mathrm{Cl}^{15} \mathrm{NN}_{2} \mathrm{OS} 381.0624$; Found $381.0600\left({ }^{15} \mathrm{~N}\right.$ product)

HRMS (ESI) $m / z$ : $[\mathrm{M}+\mathrm{H}]^{+}$Calcd for $\mathrm{C}_{20} \mathrm{H}_{15} \mathrm{ClN}_{3} \mathrm{OS} 380.0624$; Found $380.0622\left({ }^{14} \mathrm{~N}\right.$ product)

\section{Reference:}

1. Morrison, M. D.; Hanthorn, J. J.; Pratt, D. A. Synthesis of Pyrrolnitrin and Related Halogenated Phenylpyrroles. Org. Lett. 2009, 11, 1051-1054 
Figure S2: ${ }^{1} \mathrm{H}$ NMR spectrum of $12\left(500 \mathrm{MHz}, \mathrm{CDCl}_{3}\right)$
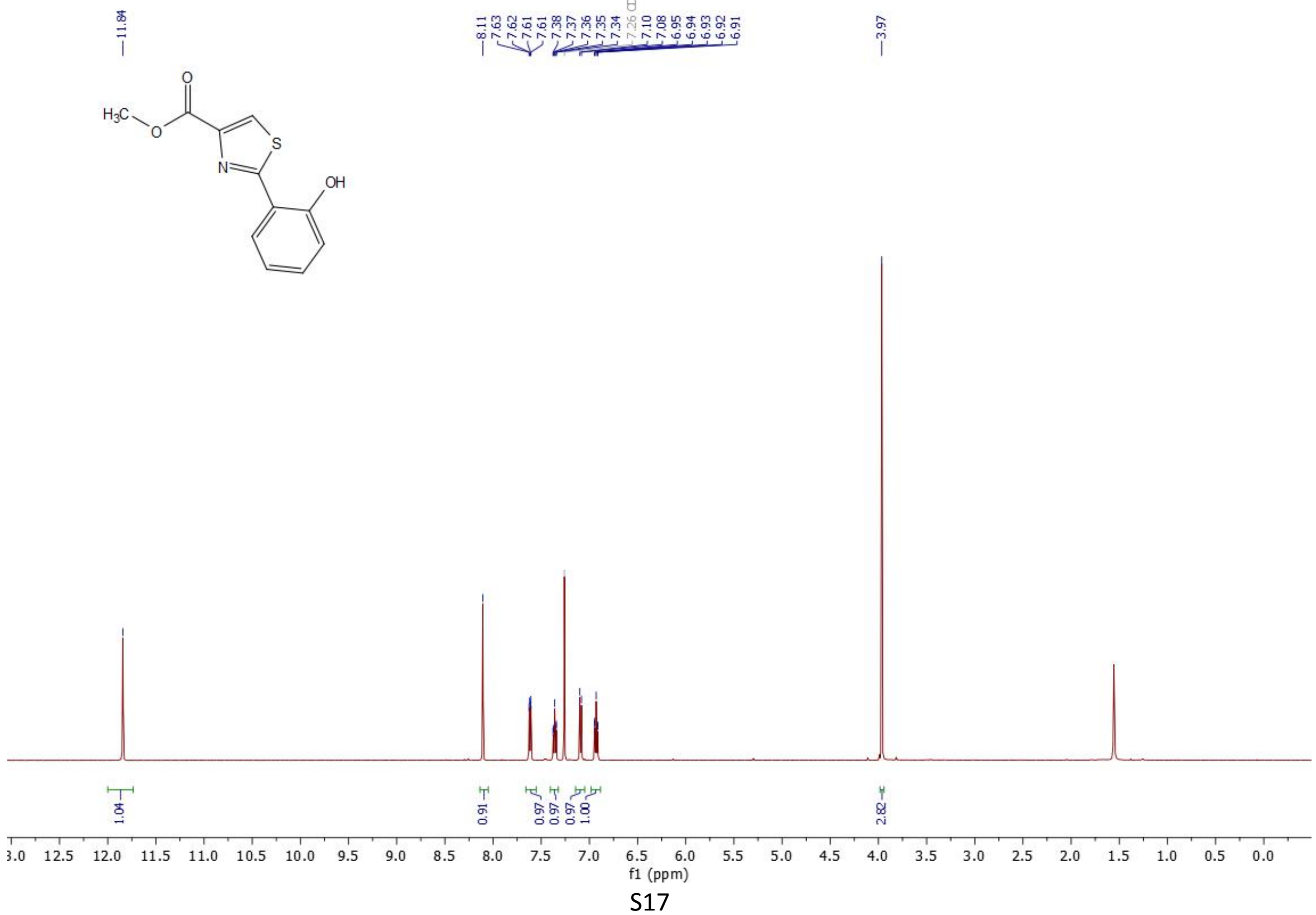

बे 
Figure S3: ${ }^{13} \mathrm{C}$ NMR spectrum of $12\left(125 \mathrm{MHz} \mathrm{CDCl}_{3}\right)$

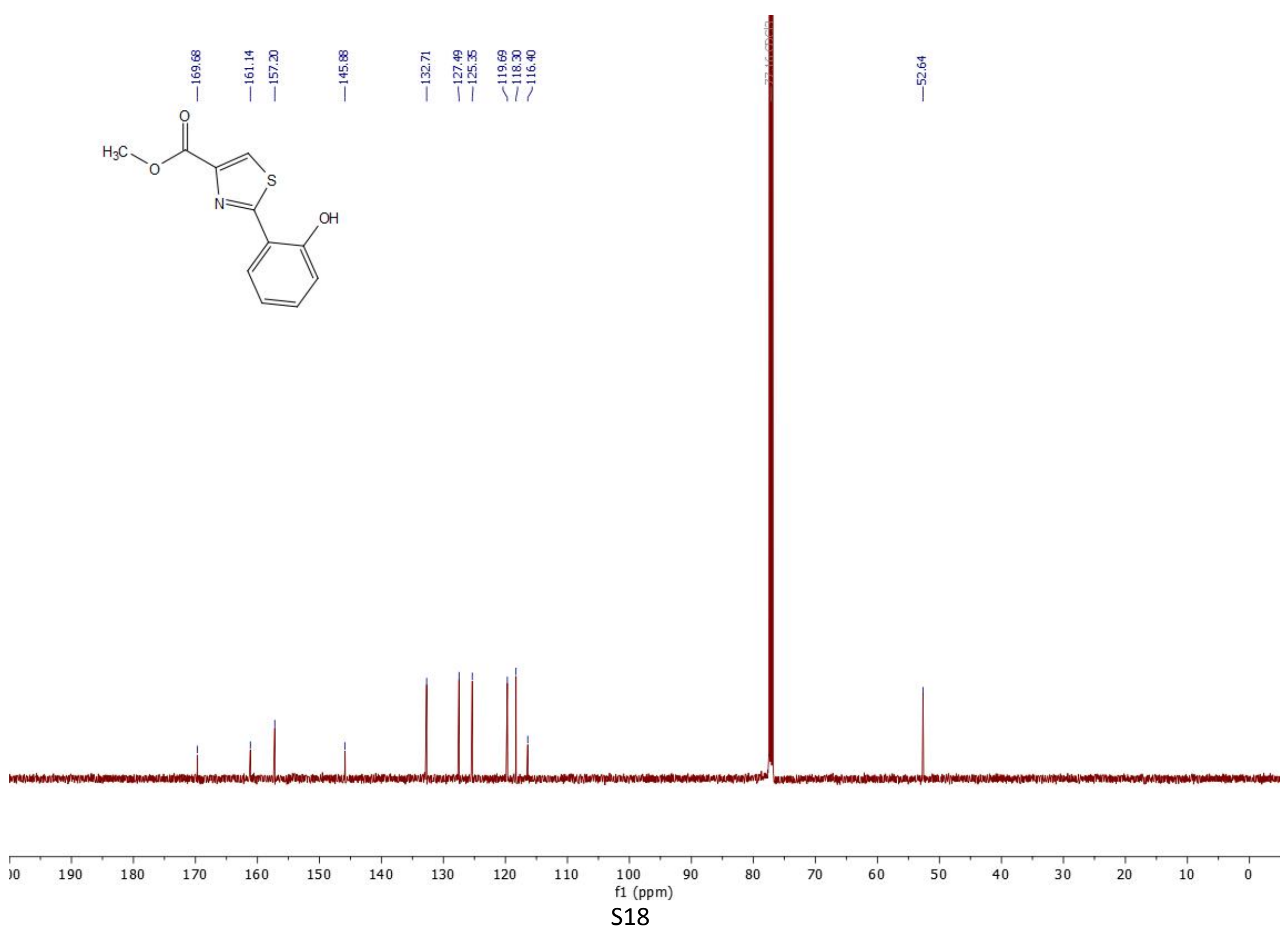


Figure S4: ${ }^{1} \mathrm{H}$ NMR spectrum of $13\left(500 \mathrm{MHz}, \mathrm{CDCl}_{3}\right)$

$\stackrel{\text { i }}{=}$
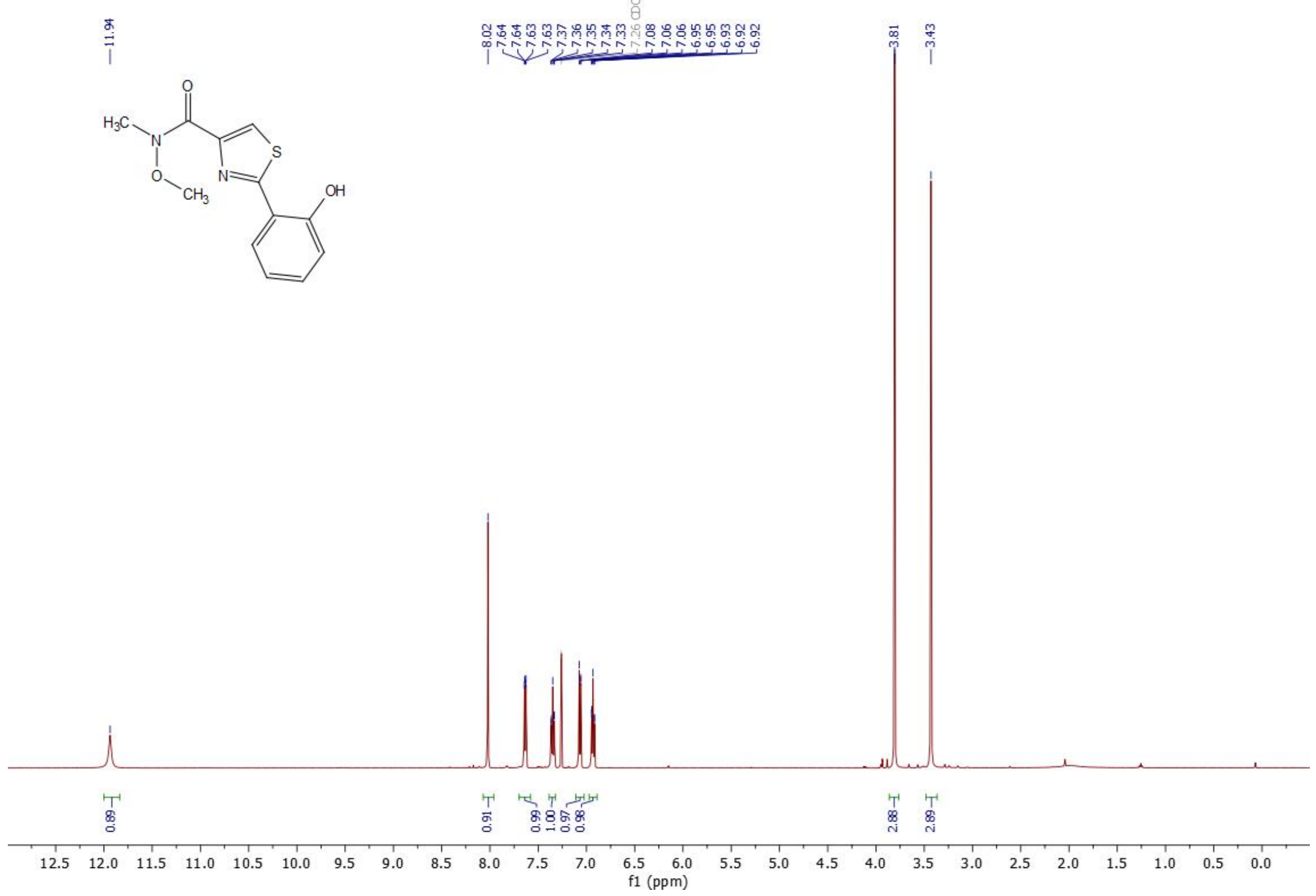

S19 
Figure S5: ${ }^{13} \mathrm{C}$ NMR spectrum of $13\left(125 \mathrm{MHz}, \mathrm{CDCl}_{3}\right)$

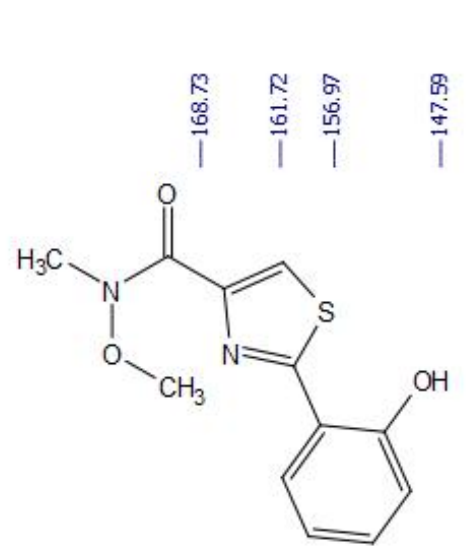

뭉

స్య

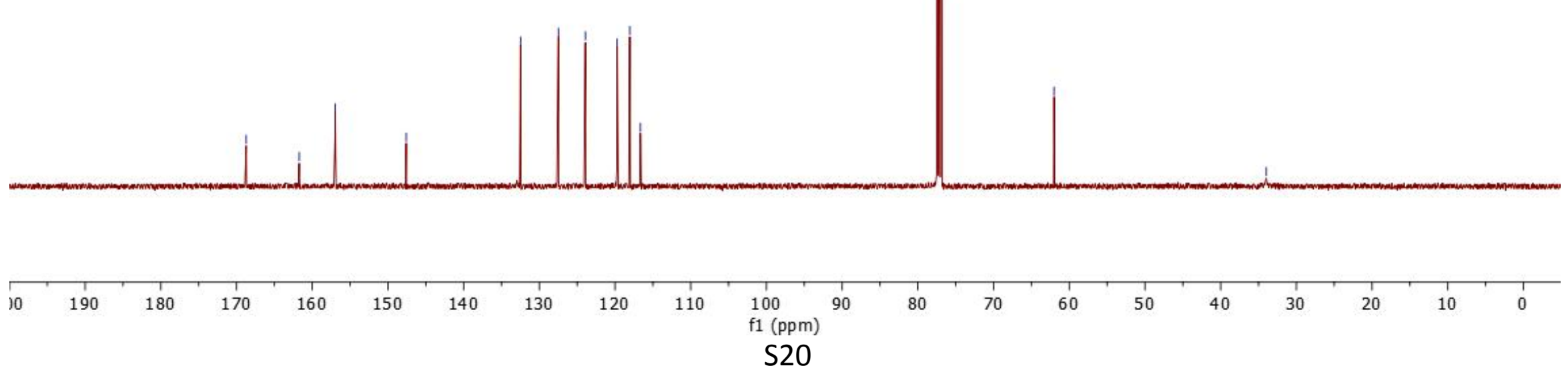


Figure S6: ${ }^{1} \mathrm{H} \mathrm{NMR}$ spectrum of $7\left(500 \mathrm{MHz}, \mathrm{CDCl}_{3}\right)$

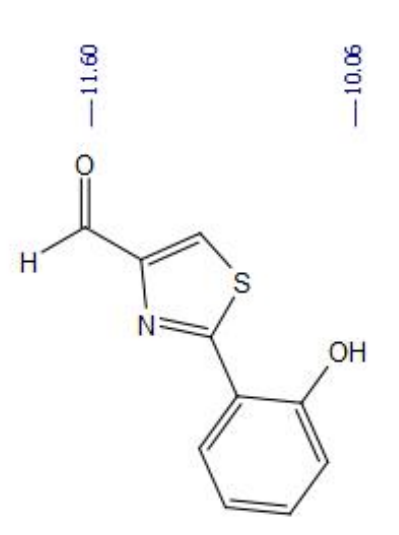

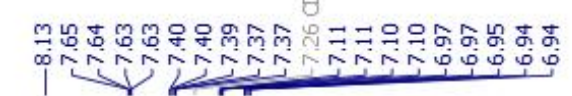

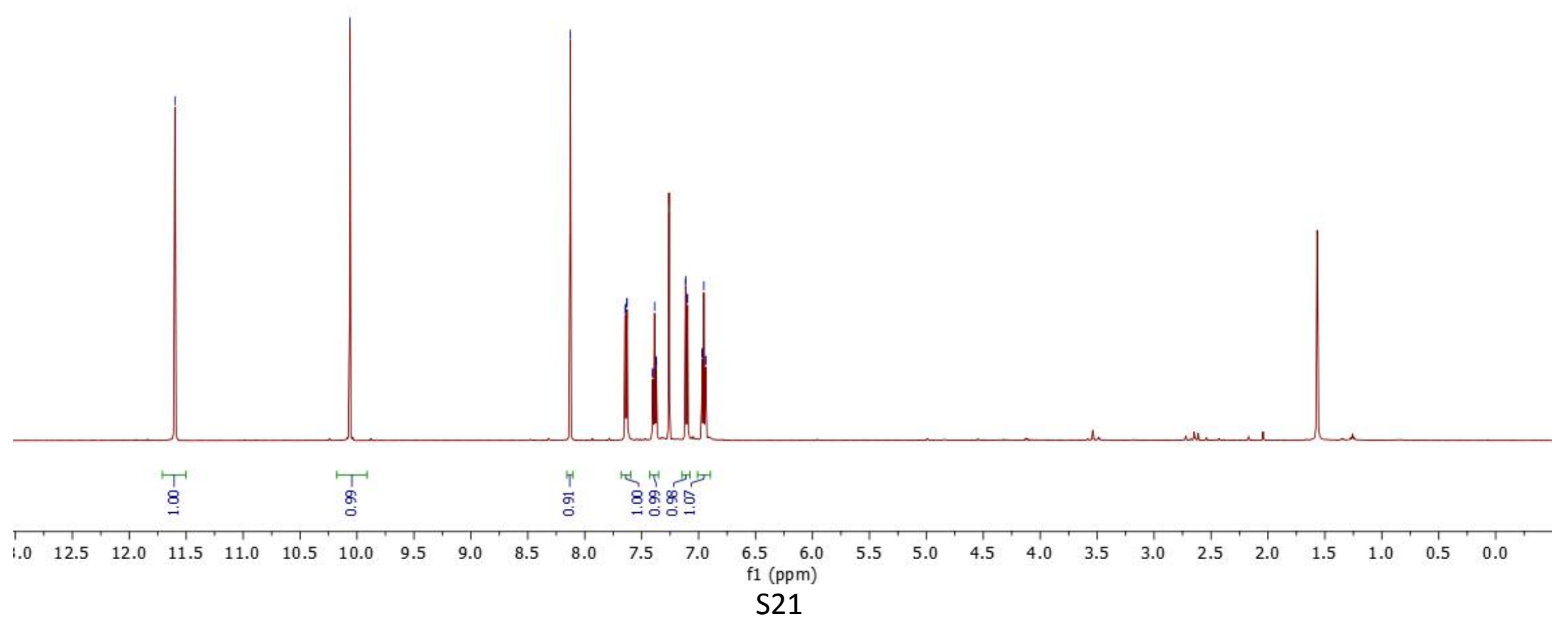


Figure S7: ${ }^{13} \mathrm{C}$ NMR spectrum of $7\left(125 \mathrm{MHz}, \mathrm{CDCl}_{3}\right)$
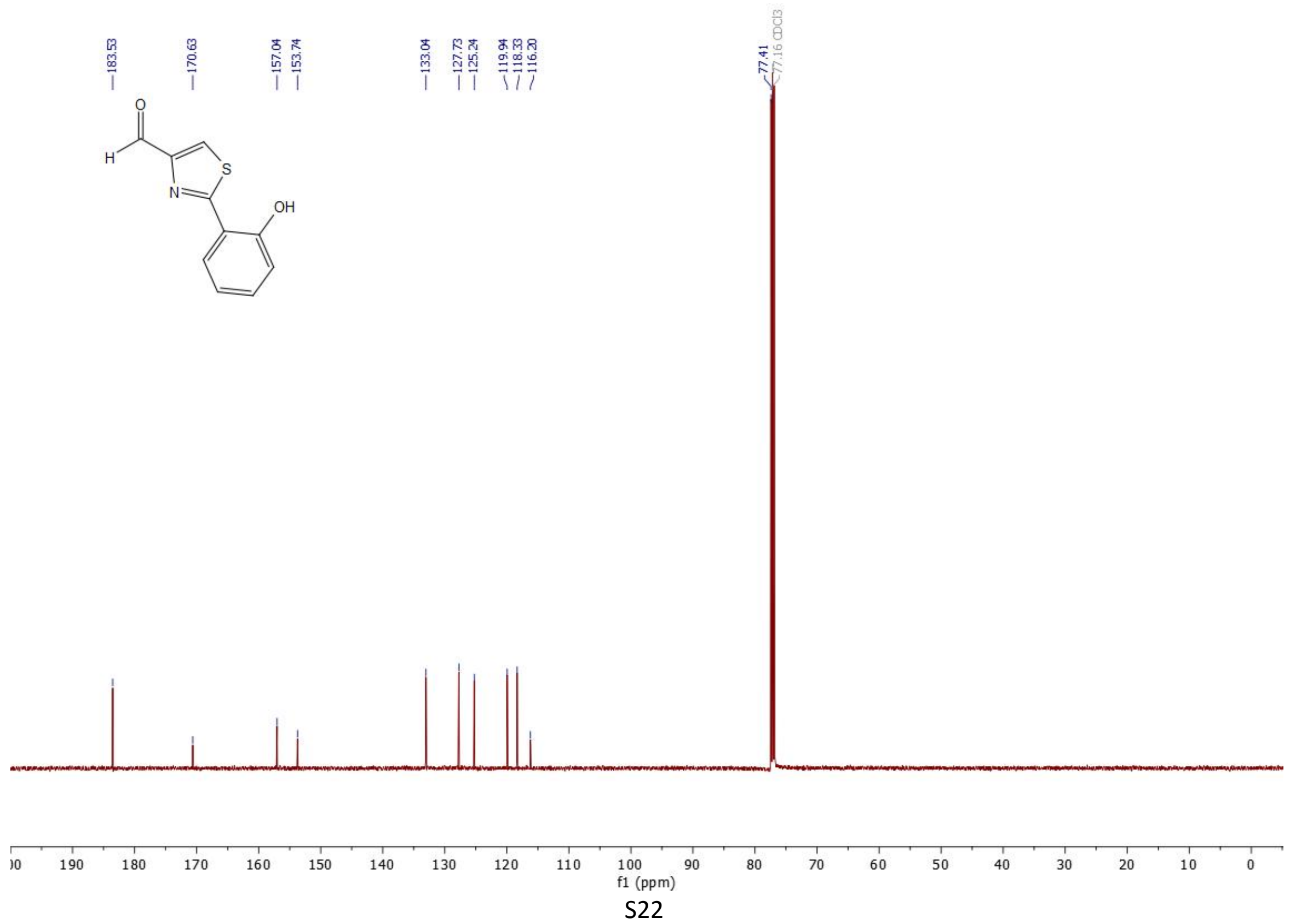
Figure S8: ${ }^{1} \mathrm{H}$ NMR spectrum of $18\left(500 \mathrm{MHz}^{\left.-\mathrm{CDCl}_{3}\right)}\right.$

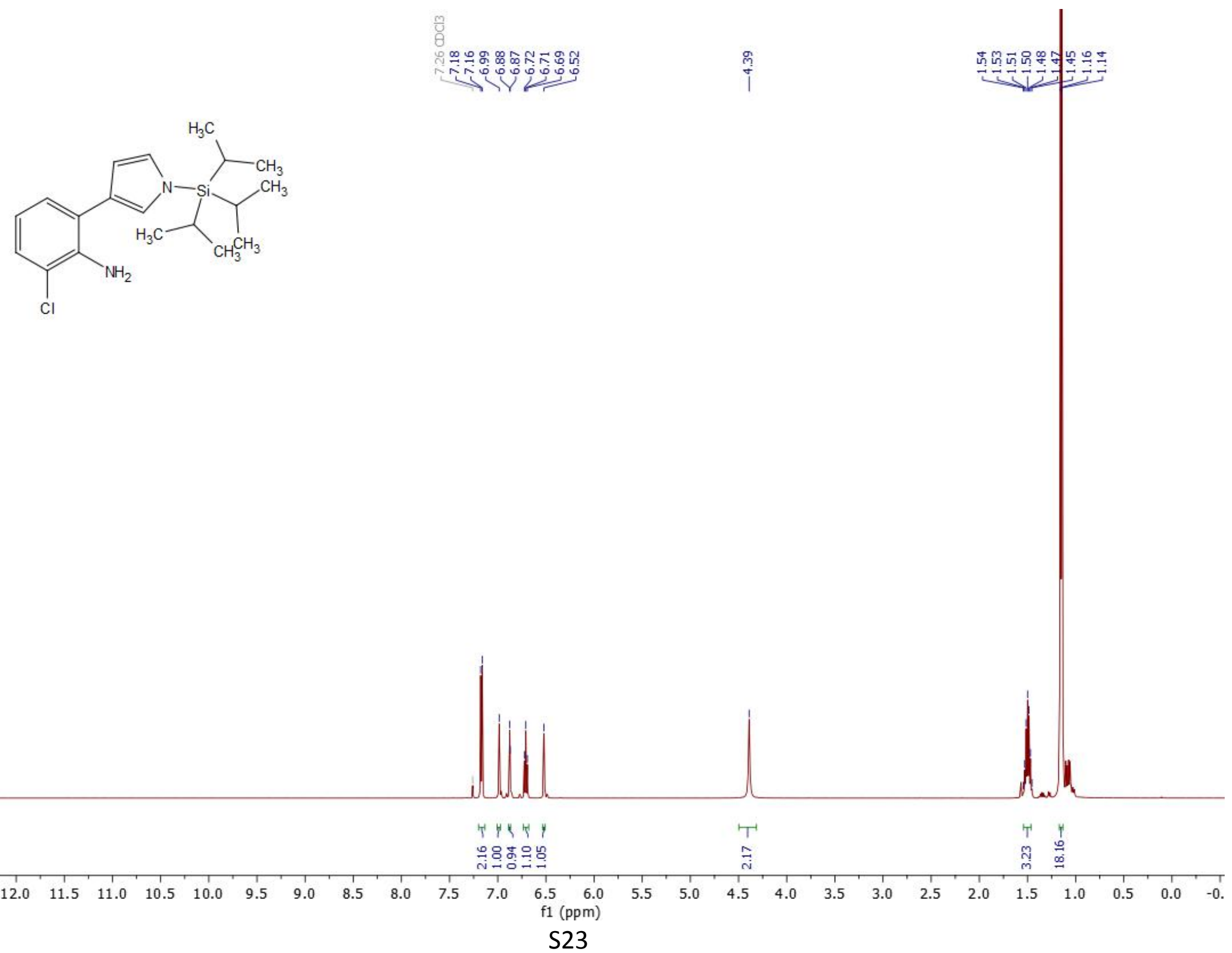


Figure S9: ${ }^{13} \mathrm{C}$ NMR spectrum of $18\left(125 \mathrm{MHz}, \mathrm{CDCl}_{3}\right)$
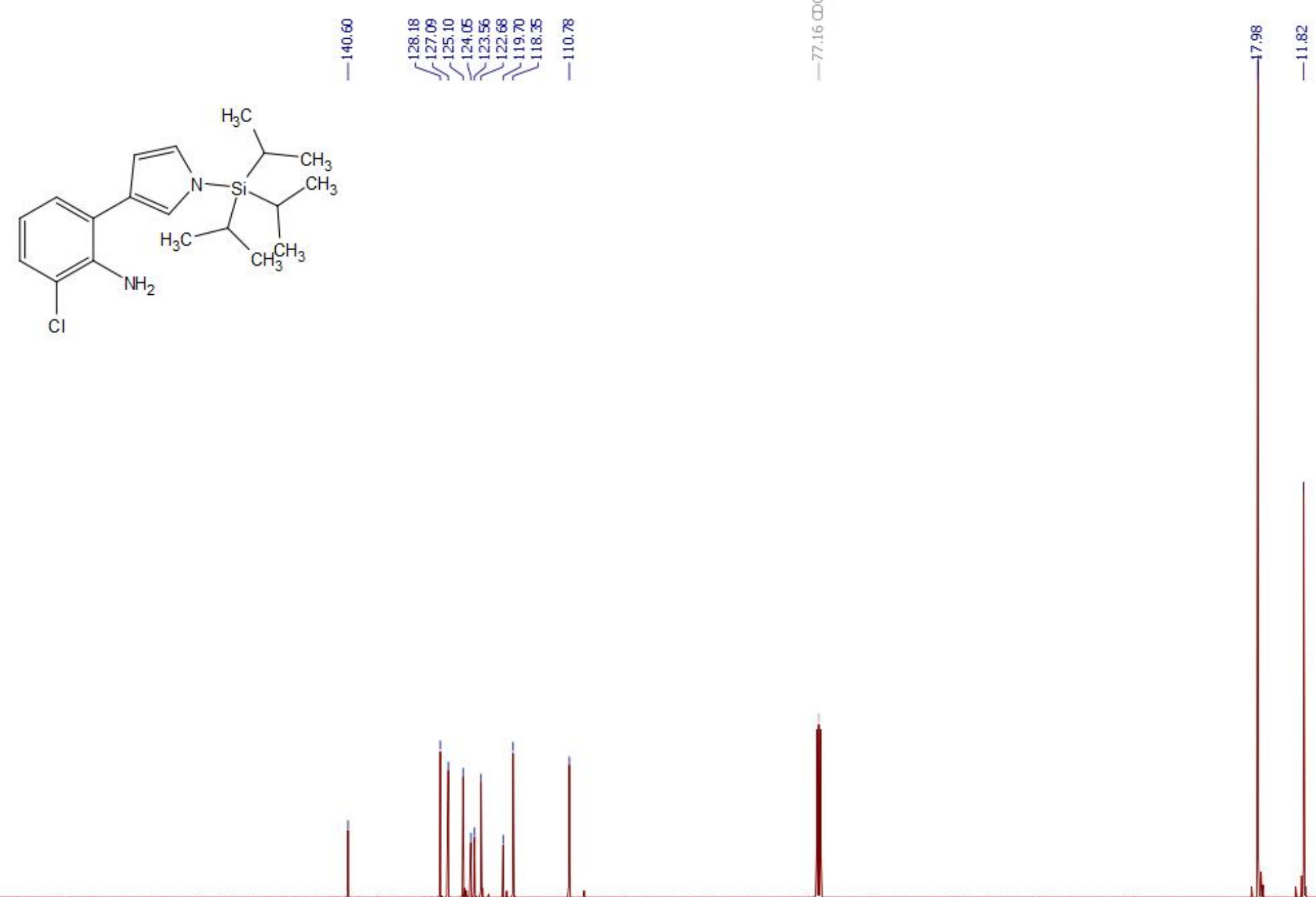

30

190

180

170

160

150

140

130

120 
Figure S10: ${ }^{1} \mathrm{H}$ NMR spectrum of $22\left(500 \mathrm{MHz}, \mathrm{CDCl}_{3}\right)$
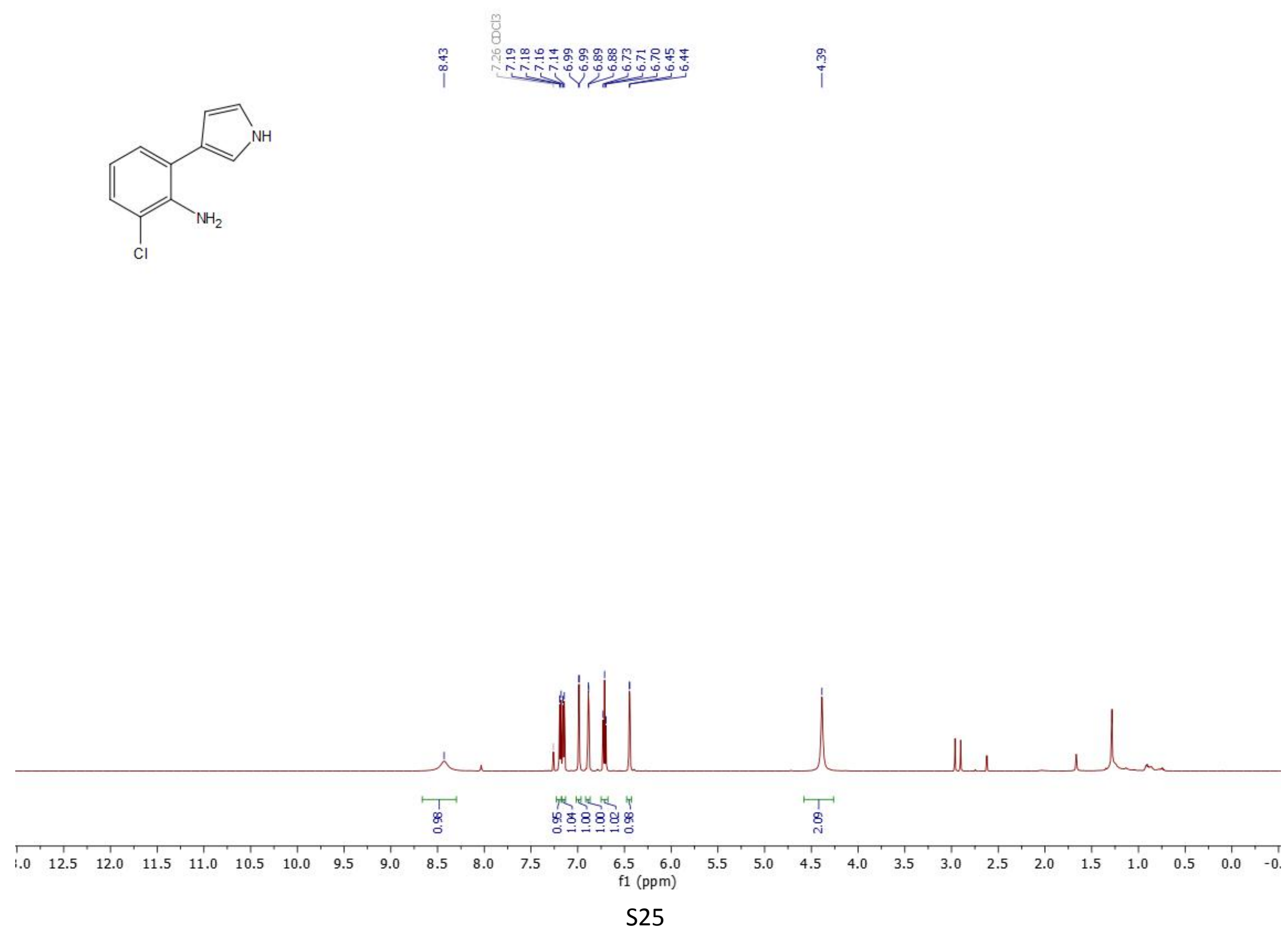
Figure S11: ${ }^{13} \mathrm{C}$ NMR spectrum of $22\left(125 \mathrm{MHz}, \mathrm{CDCl}_{3}\right)$
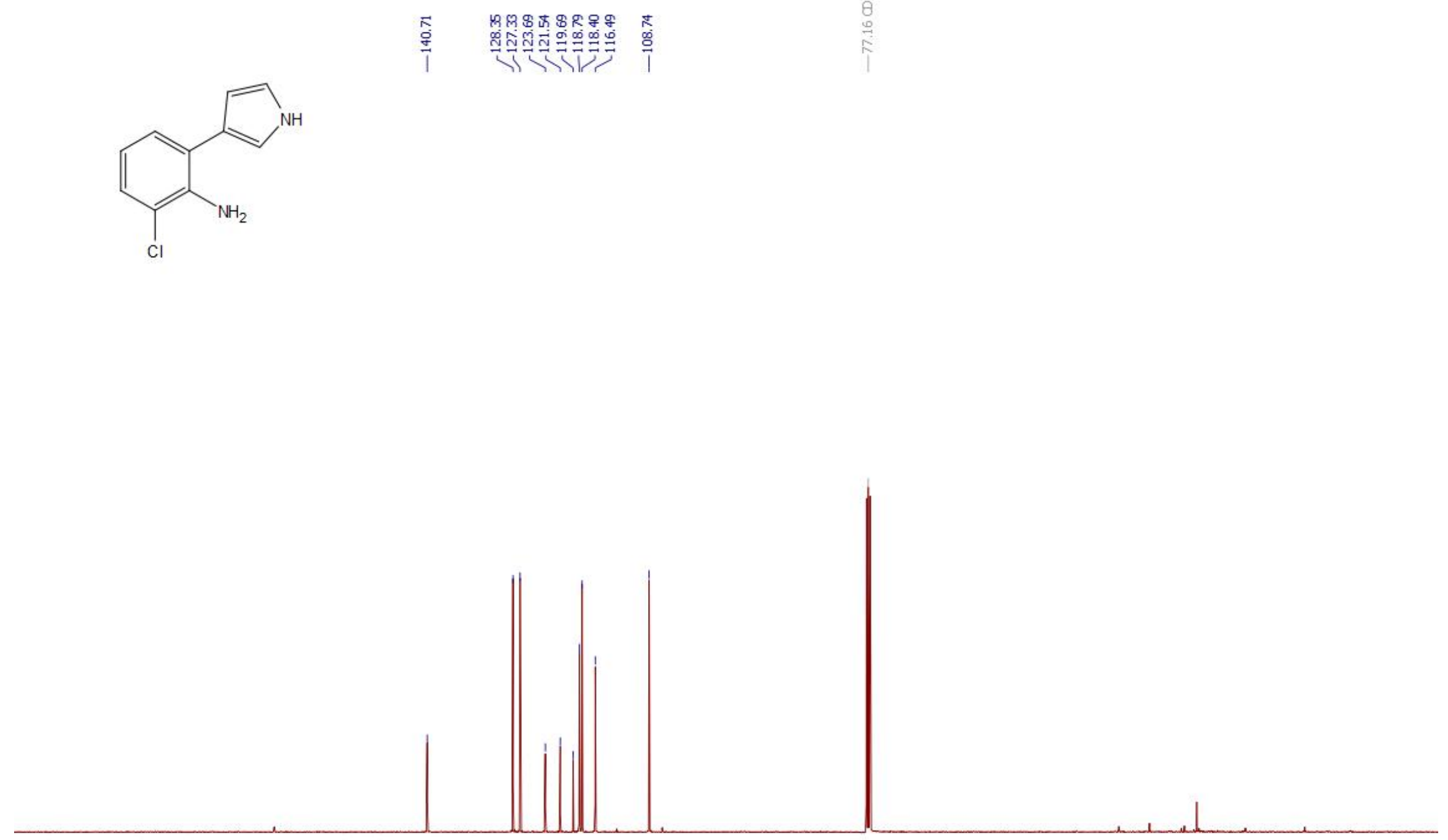
Figure S12: ${ }^{1} \mathrm{H}$ NMR spectrum of pyonitrin $\mathrm{A} 1\left(500 \mathrm{MHz}, \mathrm{DMSO}-d_{6}\right)$

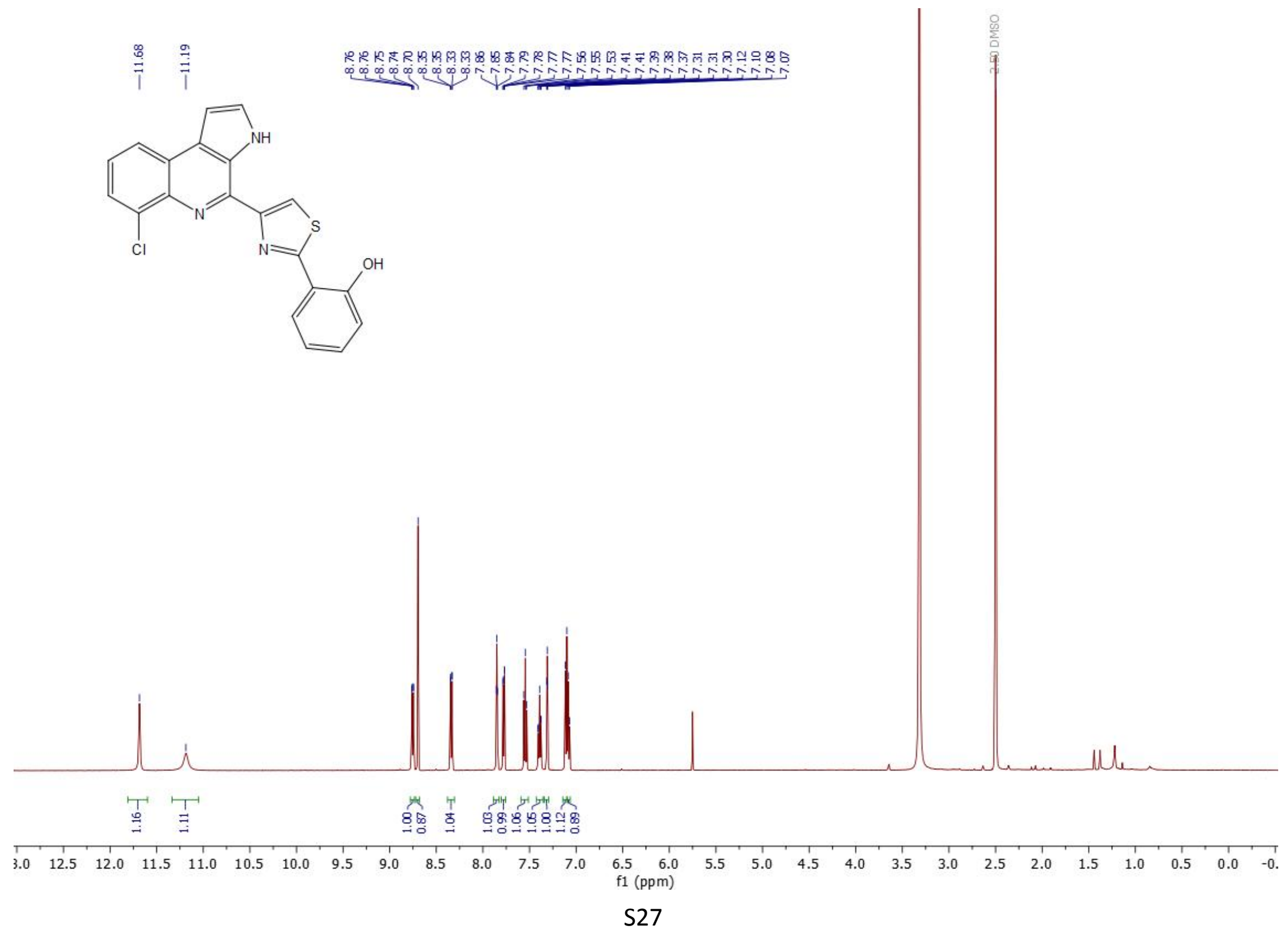


Figure S13: ${ }^{13} \mathrm{C}$ NMR spectrum of pyonitrin A $1\left(125 \mathrm{MHz}\right.$, DMSO- $\left.d_{6}\right)$
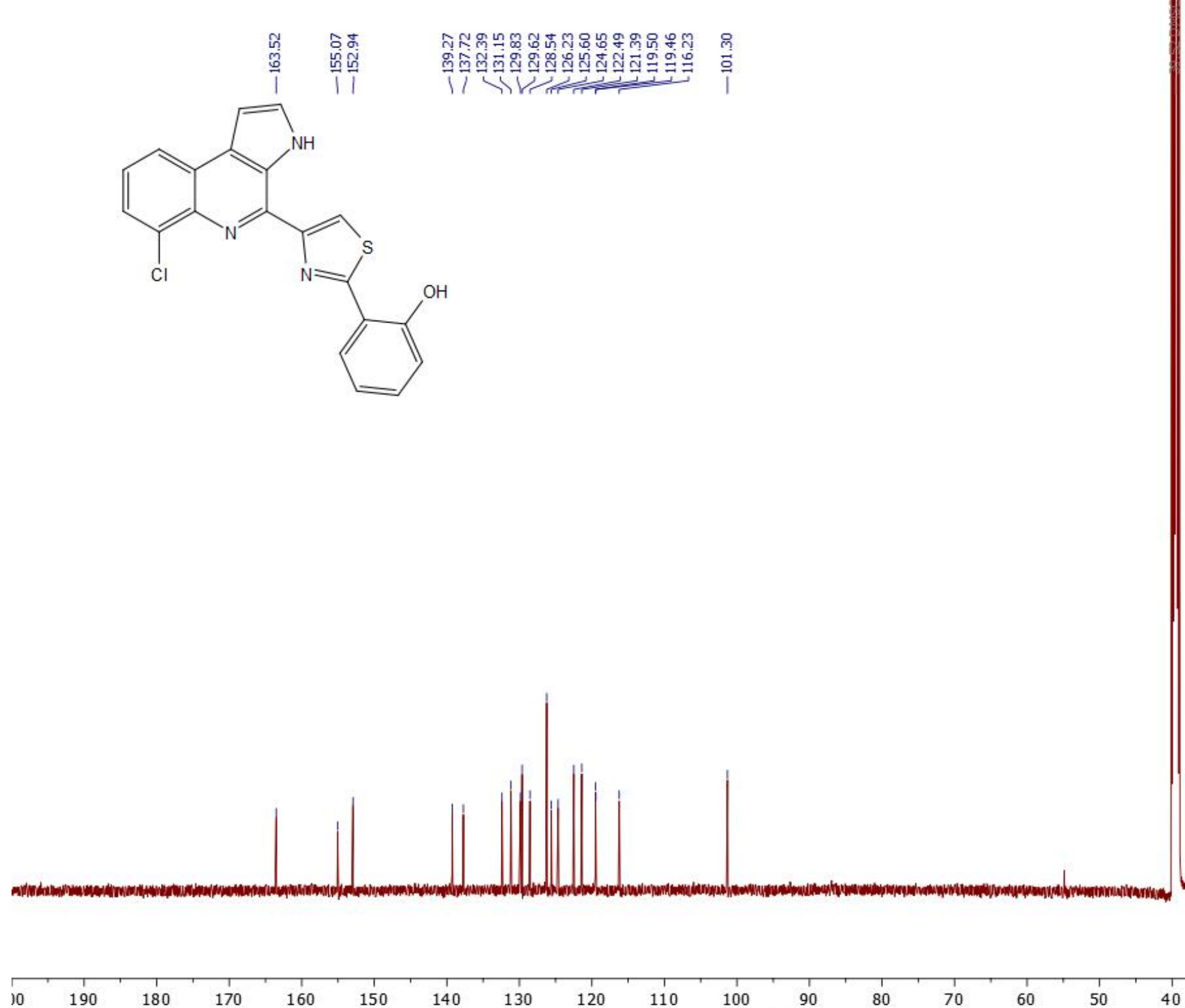

180

$170 \quad 160$

150

$140 \quad 130$

120

110 f1 (ppm) 
Figure S14: ${ }^{1} \mathrm{H}$ NMR spectrum of $19\left(500 \mathrm{MHz}, \mathrm{CDCl}_{3}\right)$

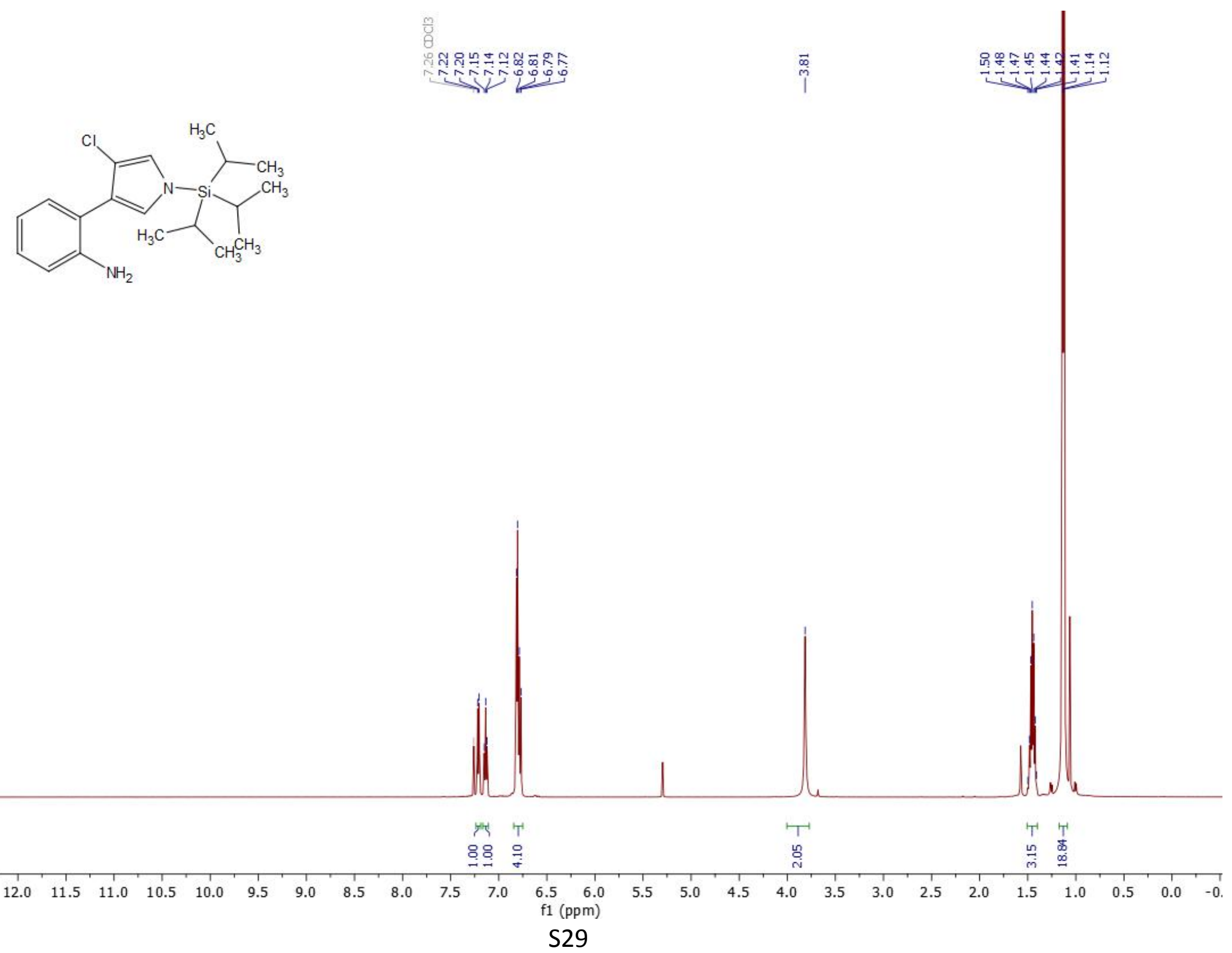


Figure S15: ${ }^{13} \mathrm{C}$ NMR spectrum of $19\left(125 \mathrm{MHz}, \mathrm{CDCl}_{3}\right)$

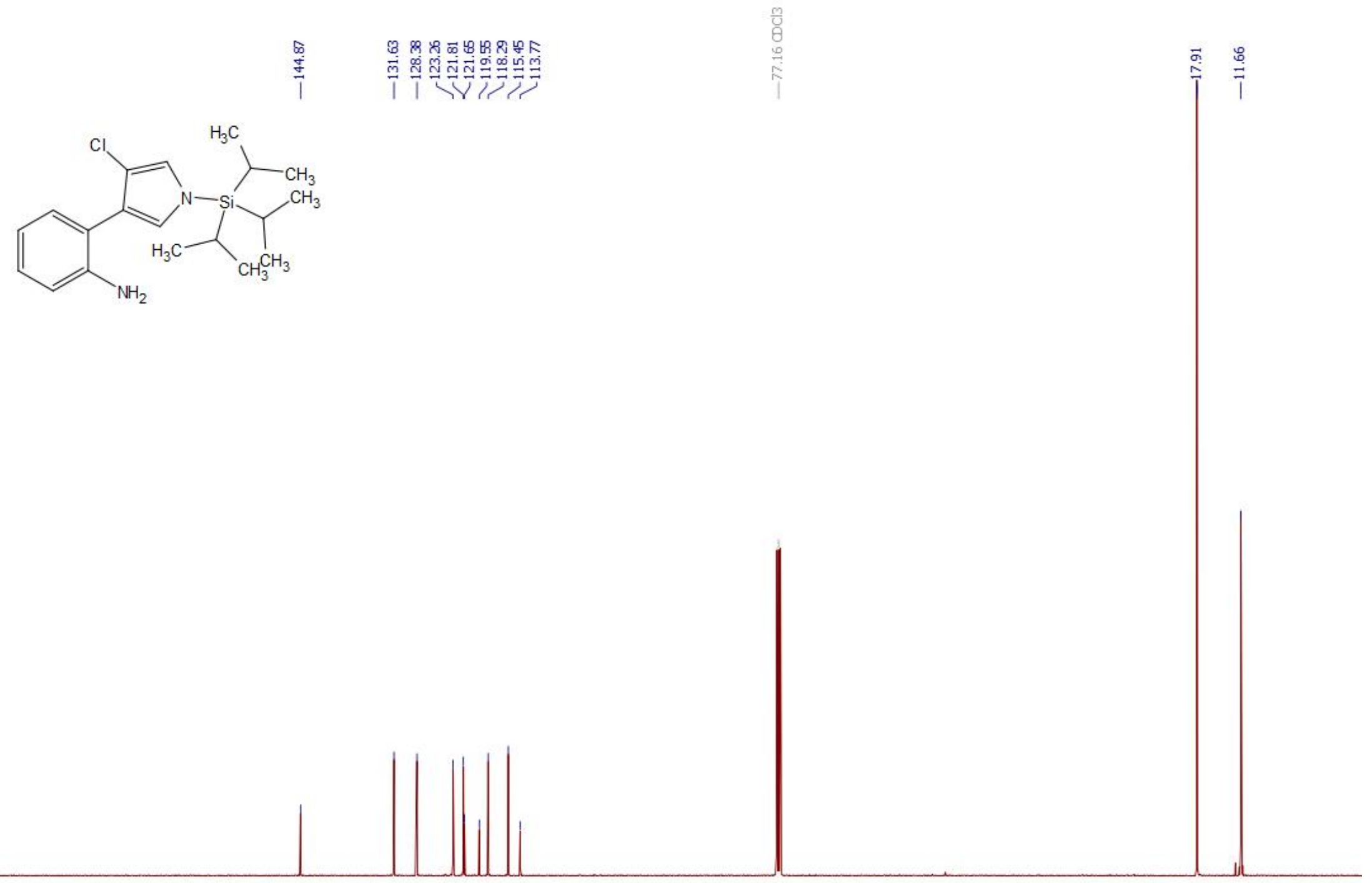

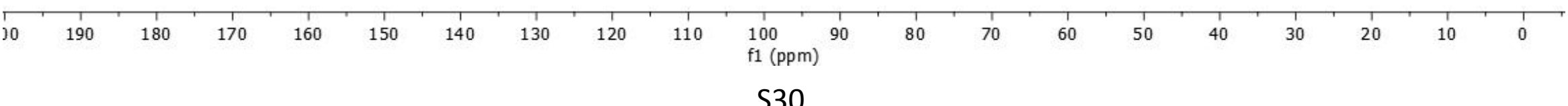


Figure S16: ${ }^{1} \mathrm{H}$ NMR spectrum of $23\left(500 \mathrm{MHz}, \mathrm{CDCl}_{3}\right)$
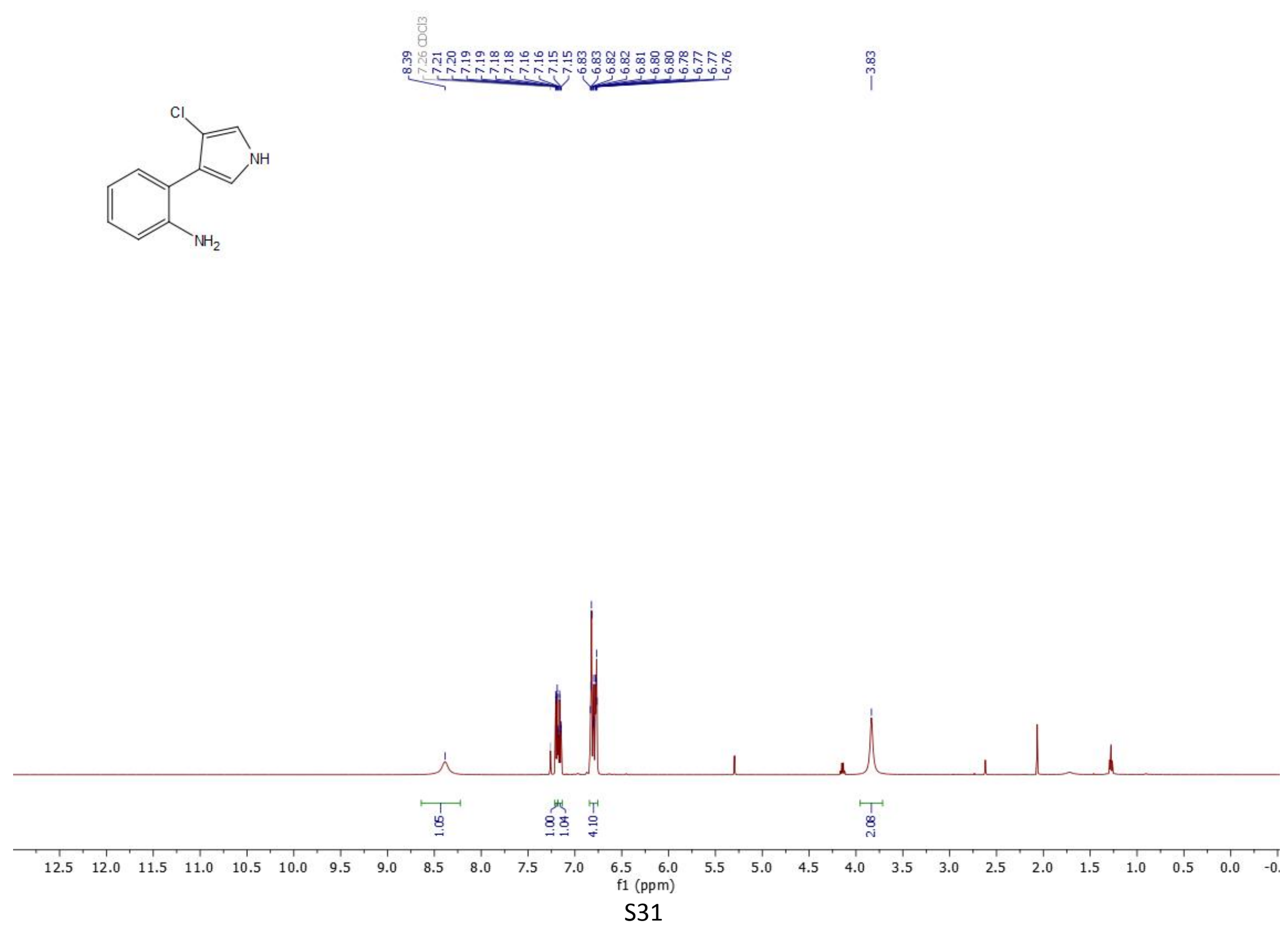
Figure S17: ${ }^{13} \mathrm{C}$ NMR spectrum of $23\left(125 \mathrm{MHz}, \mathrm{CDCl}_{3}\right)$
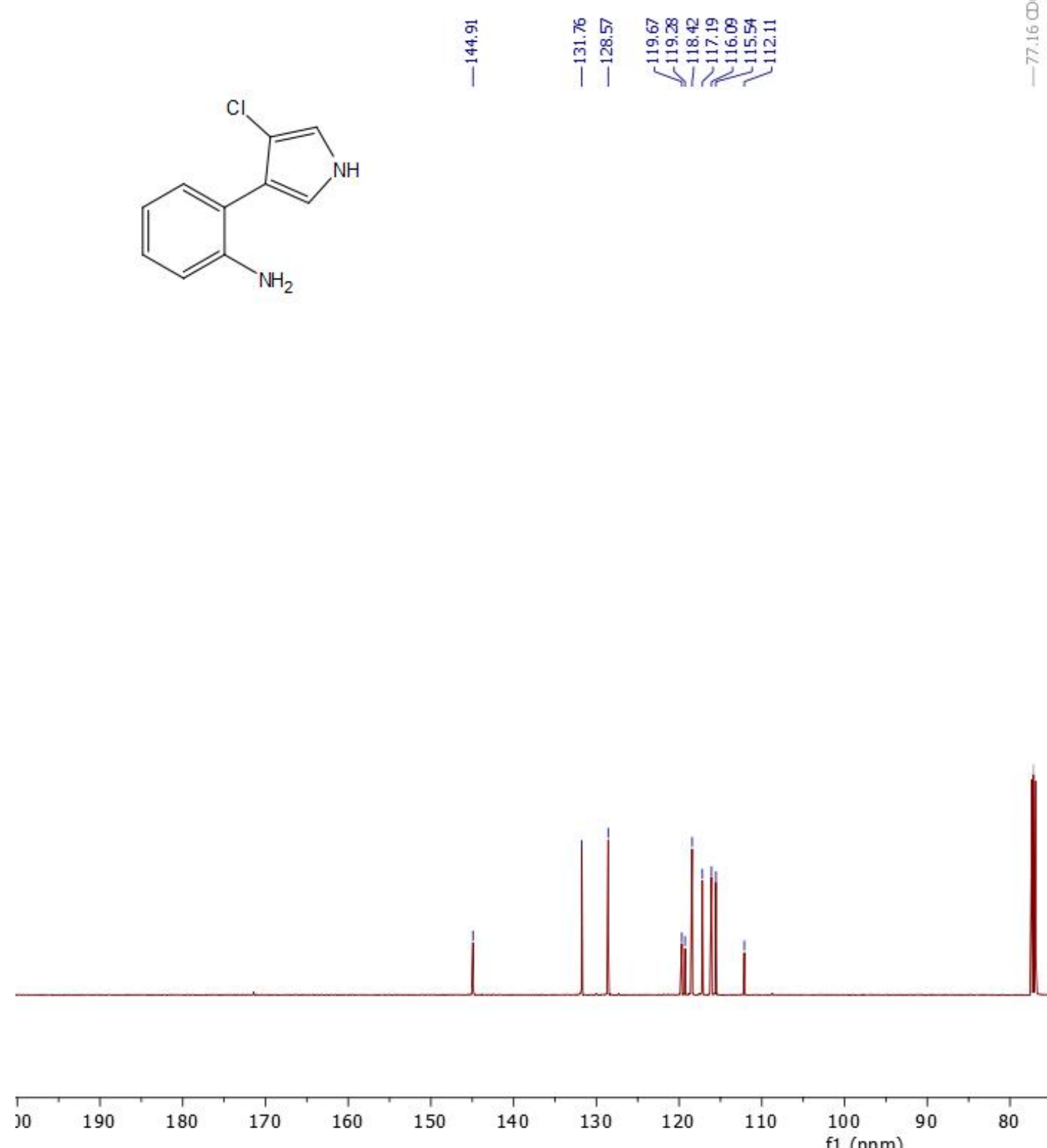

$190 \quad 180$ 
Figure S18: ${ }^{1} \mathrm{H}$ NMR spectrum of pyonitrin B (800 MHz, DMSO- $\left.d_{6}\right)$

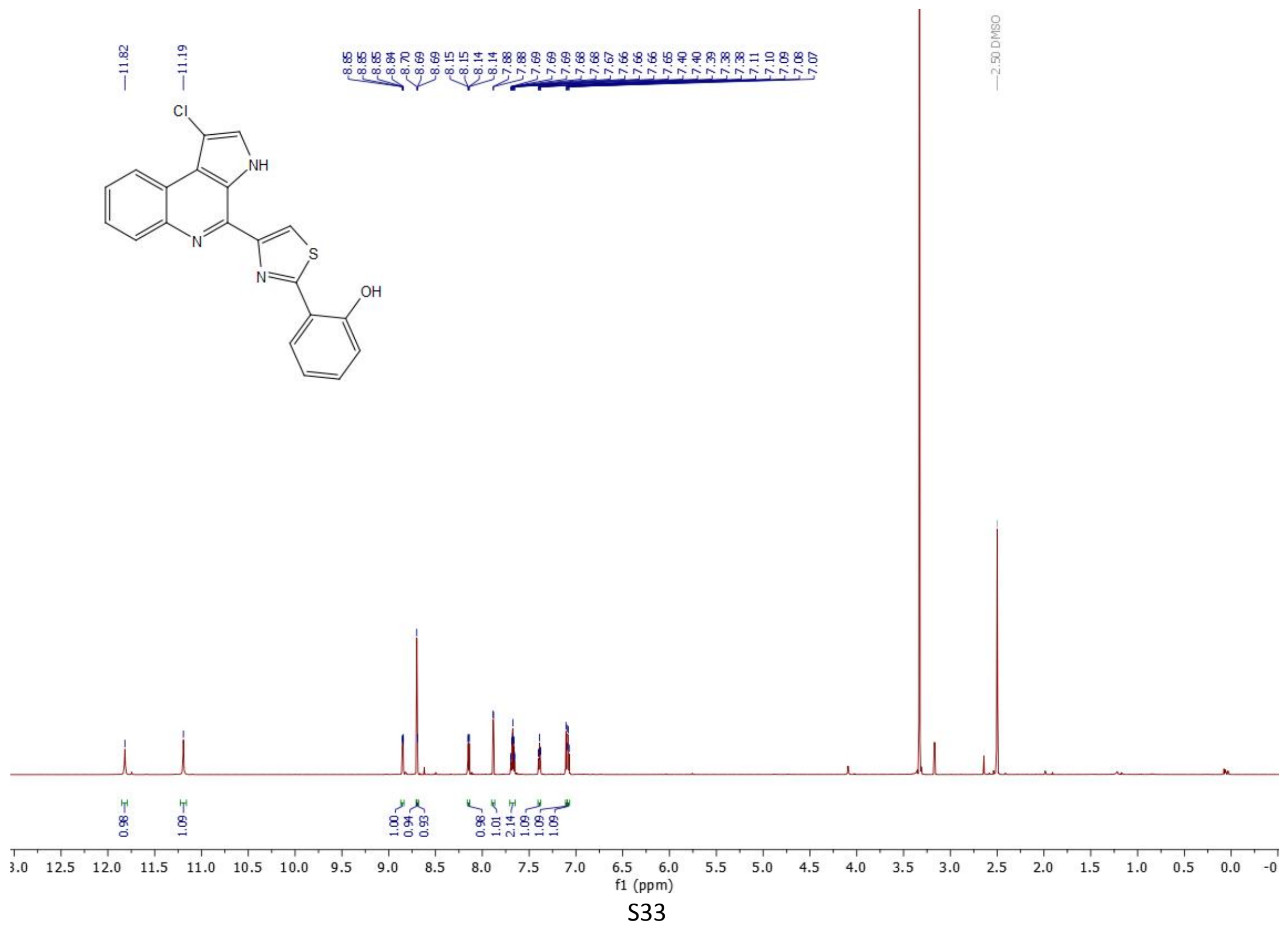


Figure S19: ${ }^{13} \mathrm{C}$ NMR spectrum of pyonitrin B $\left(200 \mathrm{MHz}, \mathrm{DMSO}-d_{6}\right)$

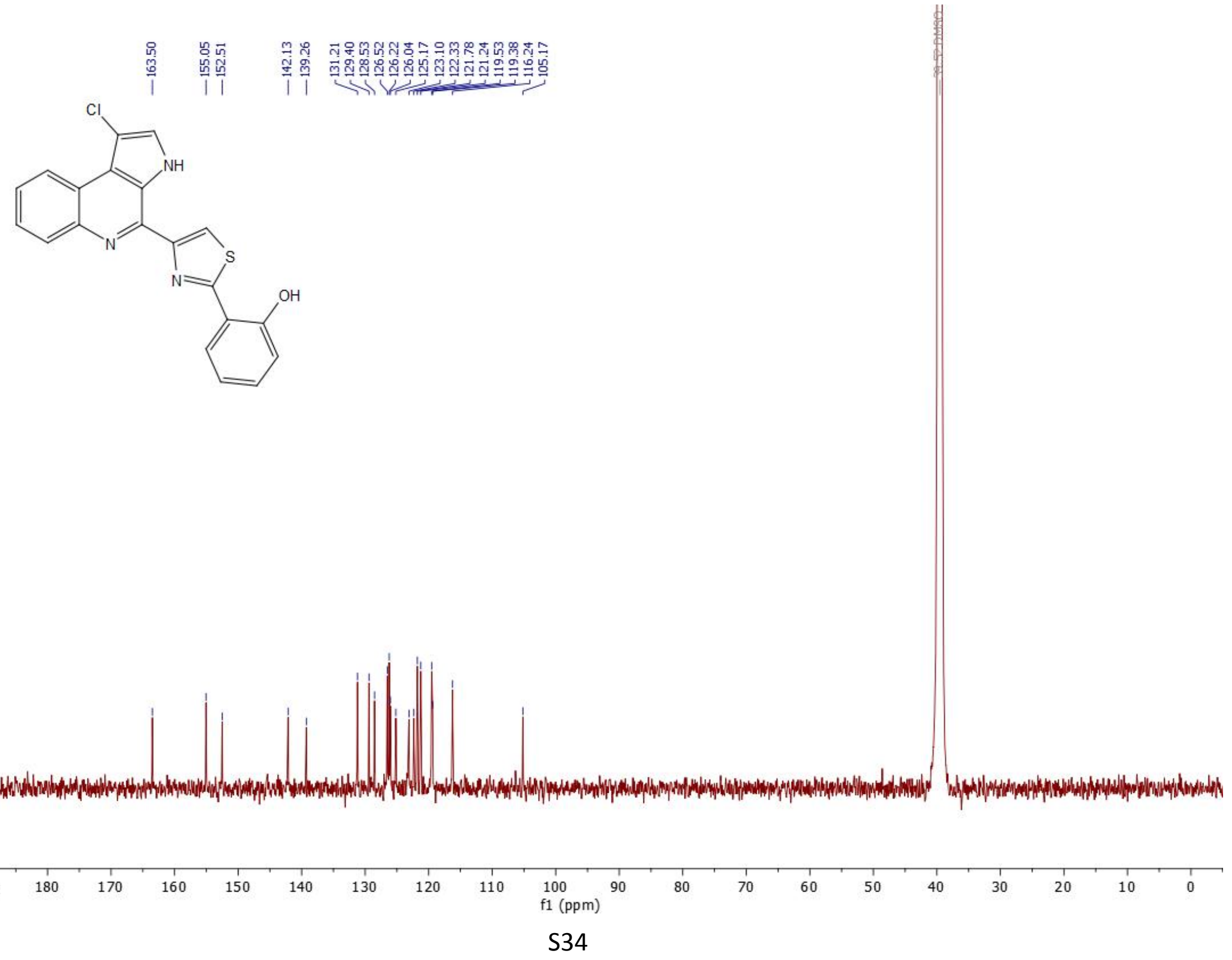


Figure S20: ${ }^{1} \mathrm{H}$ NMR spectrum of $20\left(500 \mathrm{MHz}, \mathrm{CDCl}_{3}\right)$

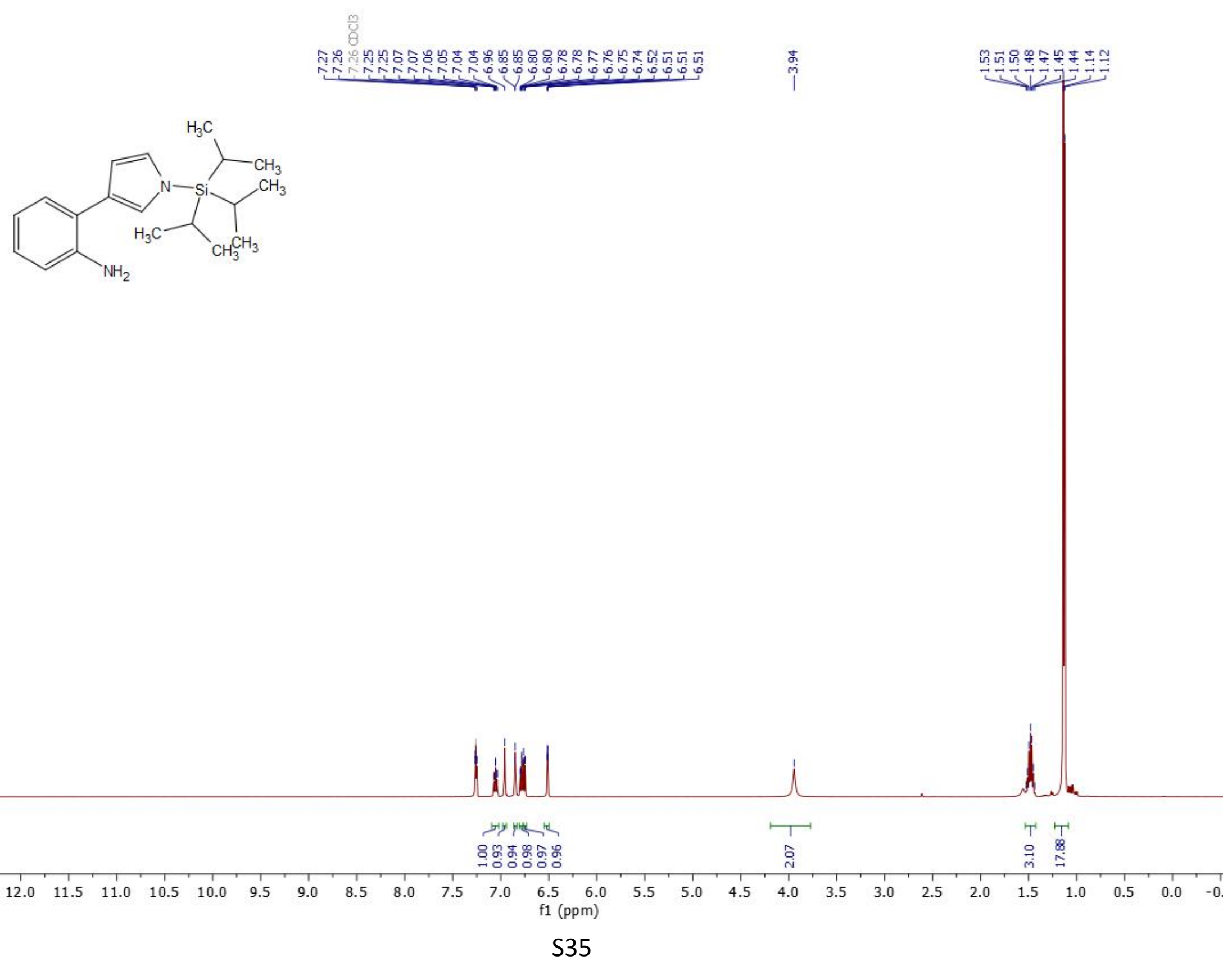


Figure S21: ${ }^{13} \mathrm{C}$ NMR spectrum of $20\left(125 \mathrm{MHz}, \mathrm{CDCl}_{3}\right)$

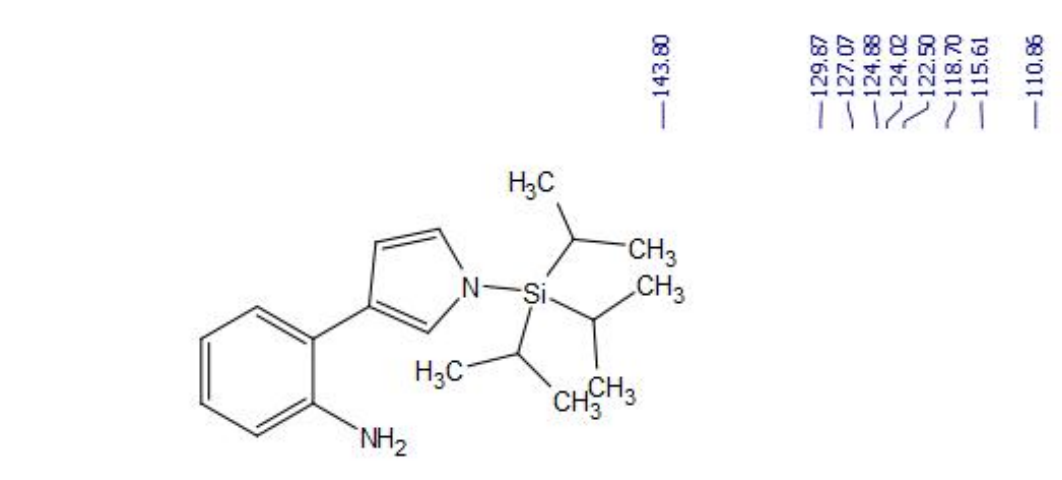

$\frac{10}{0}$
8
0
0
0
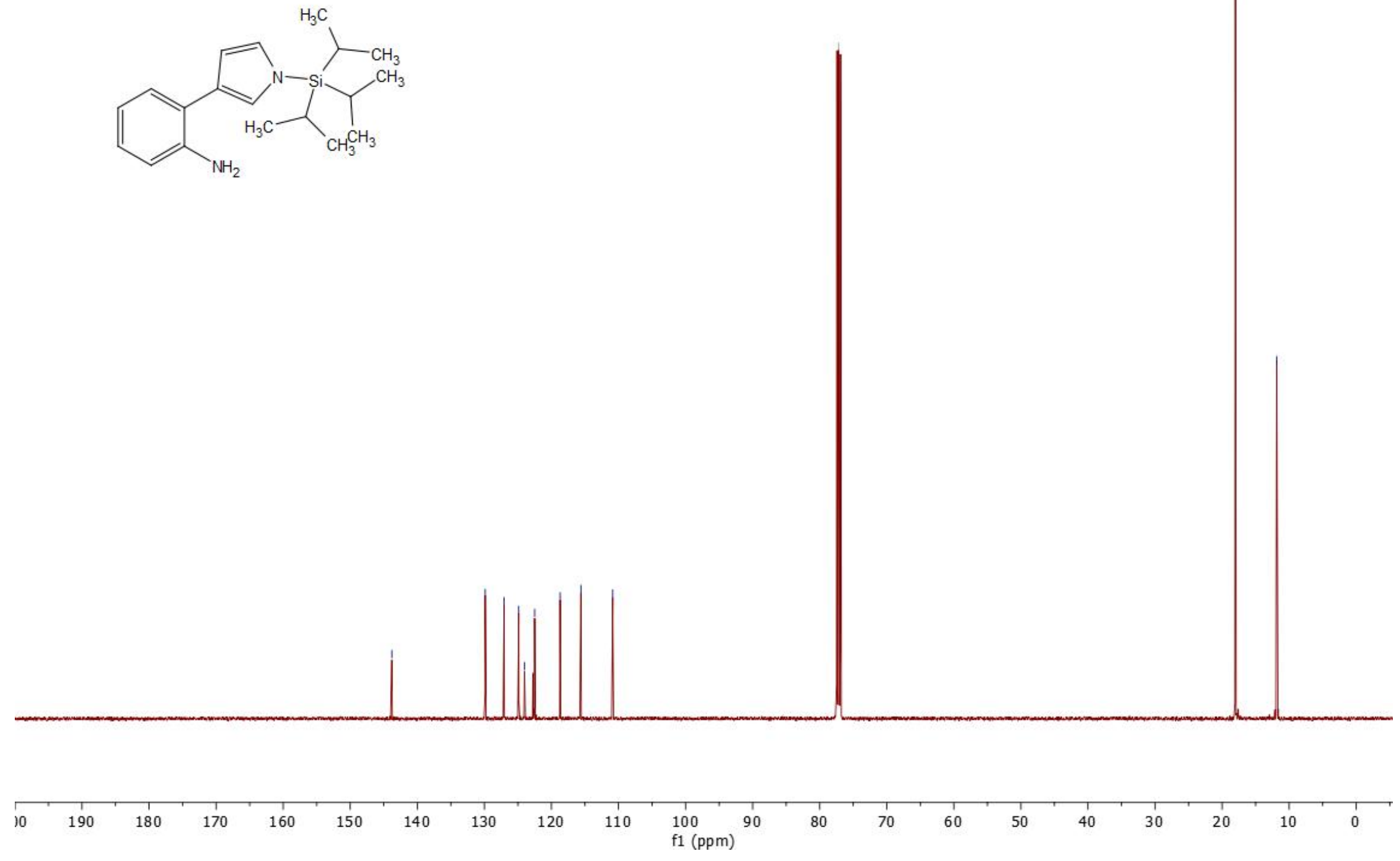

S36 
Figure S22: ${ }^{1} \mathrm{H}$ NMR spectrum of $24\left(500 \mathrm{MHz}, \mathrm{CDCl}_{3}\right)$
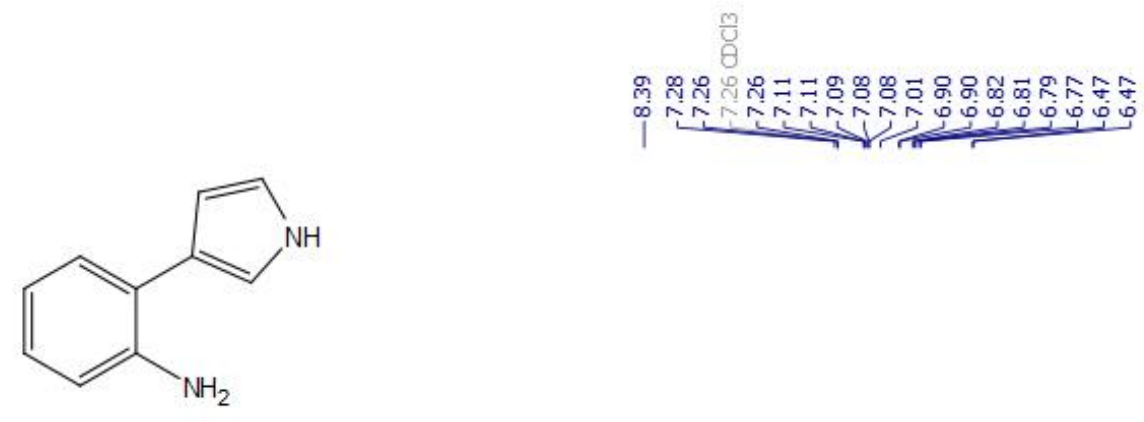

$\stackrel{\substack{n \\ i}}{i}$

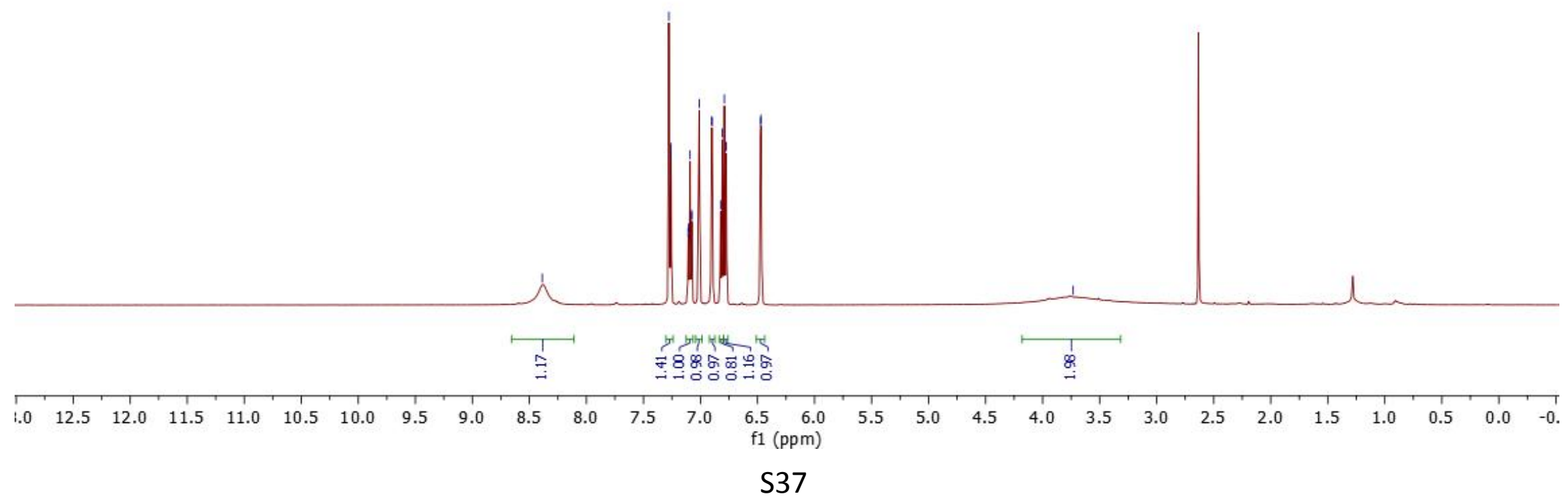


Figure S23: ${ }^{13} \mathrm{C}$ NMR spectrum of $24\left(125 \mathrm{MHz}, \mathrm{CDCl}_{3}\right)$
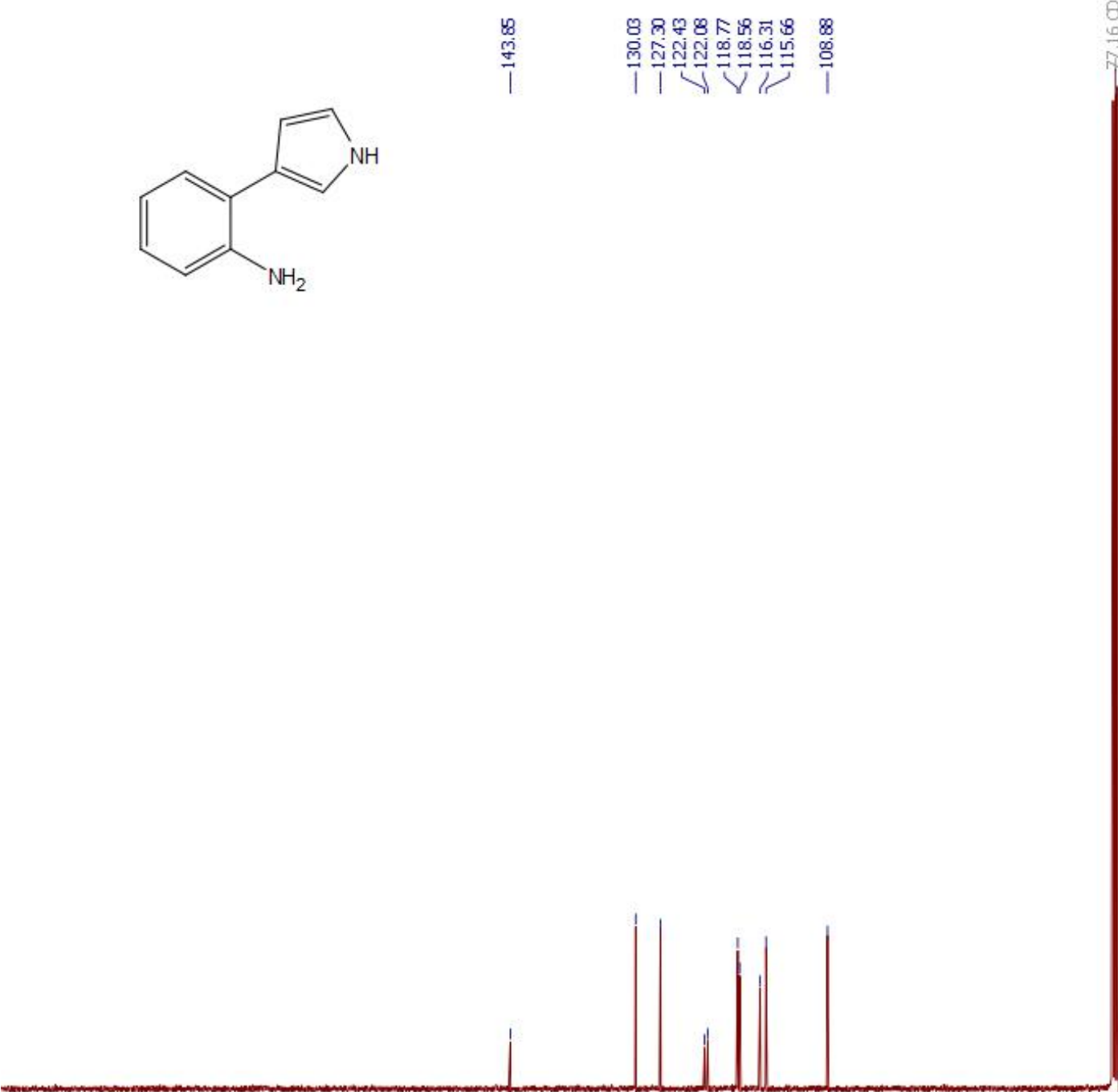

$10 \quad 190$

180

$70 \quad 160$

$150 \quad 140$

130

120

110 
Figure S24: ${ }^{1} \mathrm{H}$ NMR spectrum of pyonitrin $\mathrm{C}\left(800 \mathrm{MHz}, \mathrm{DMDO}-d_{6}\right)$

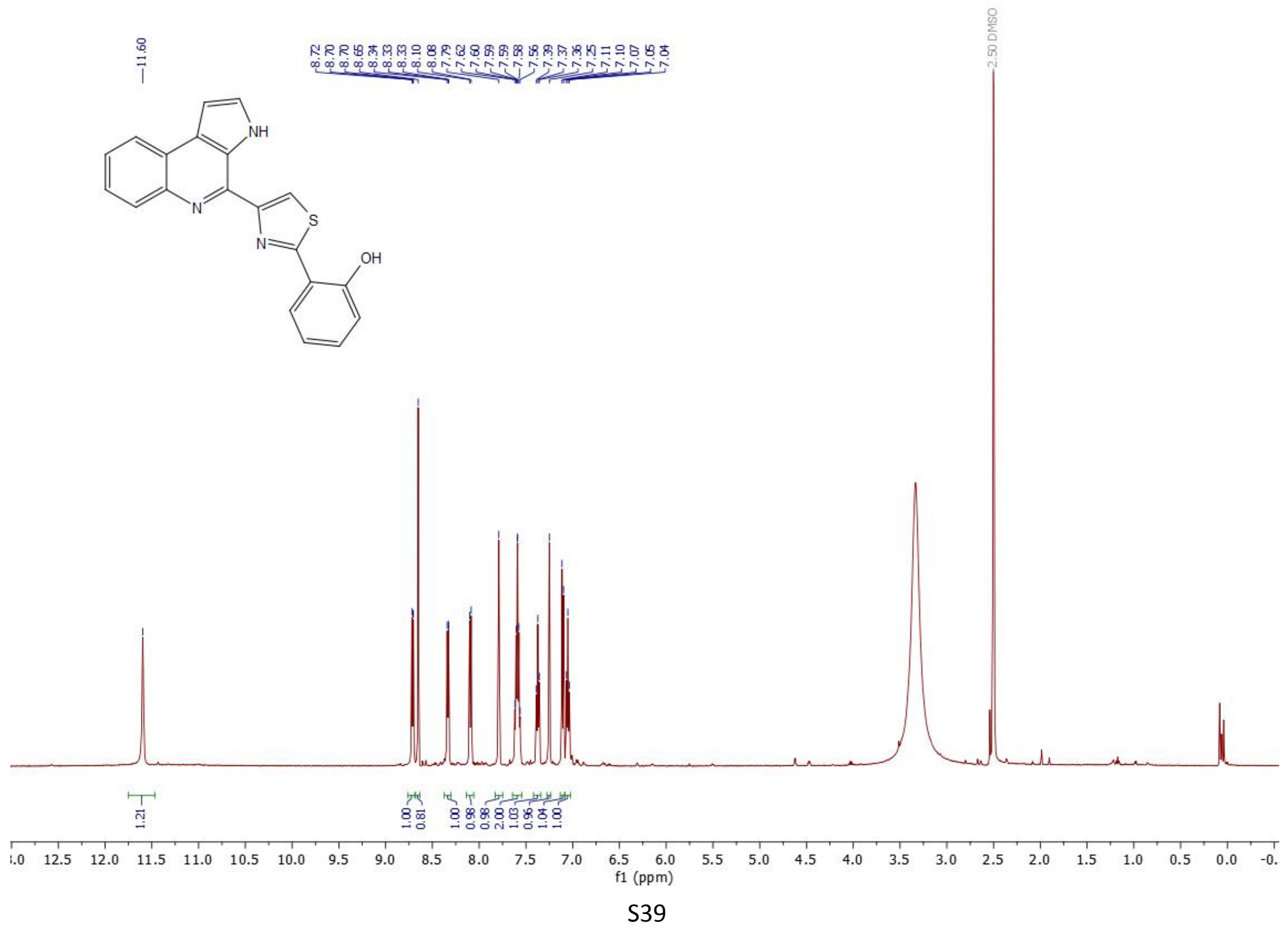


Figure S25: ${ }^{13} \mathrm{C}$ NMR spectrum of pyonitrin C (200 MHz, DMDO- $\left.d_{6}\right)$

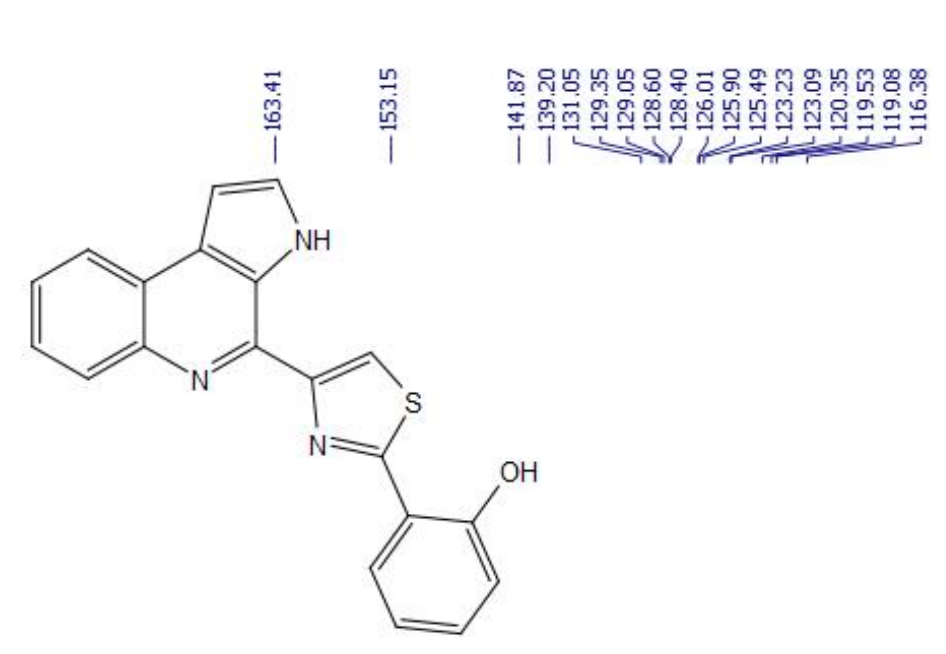


Figure S26: ${ }^{1} \mathrm{H}$ NMR spectrum of $21\left(500 \mathrm{MHz}, \mathrm{CDCl}_{3}\right)$
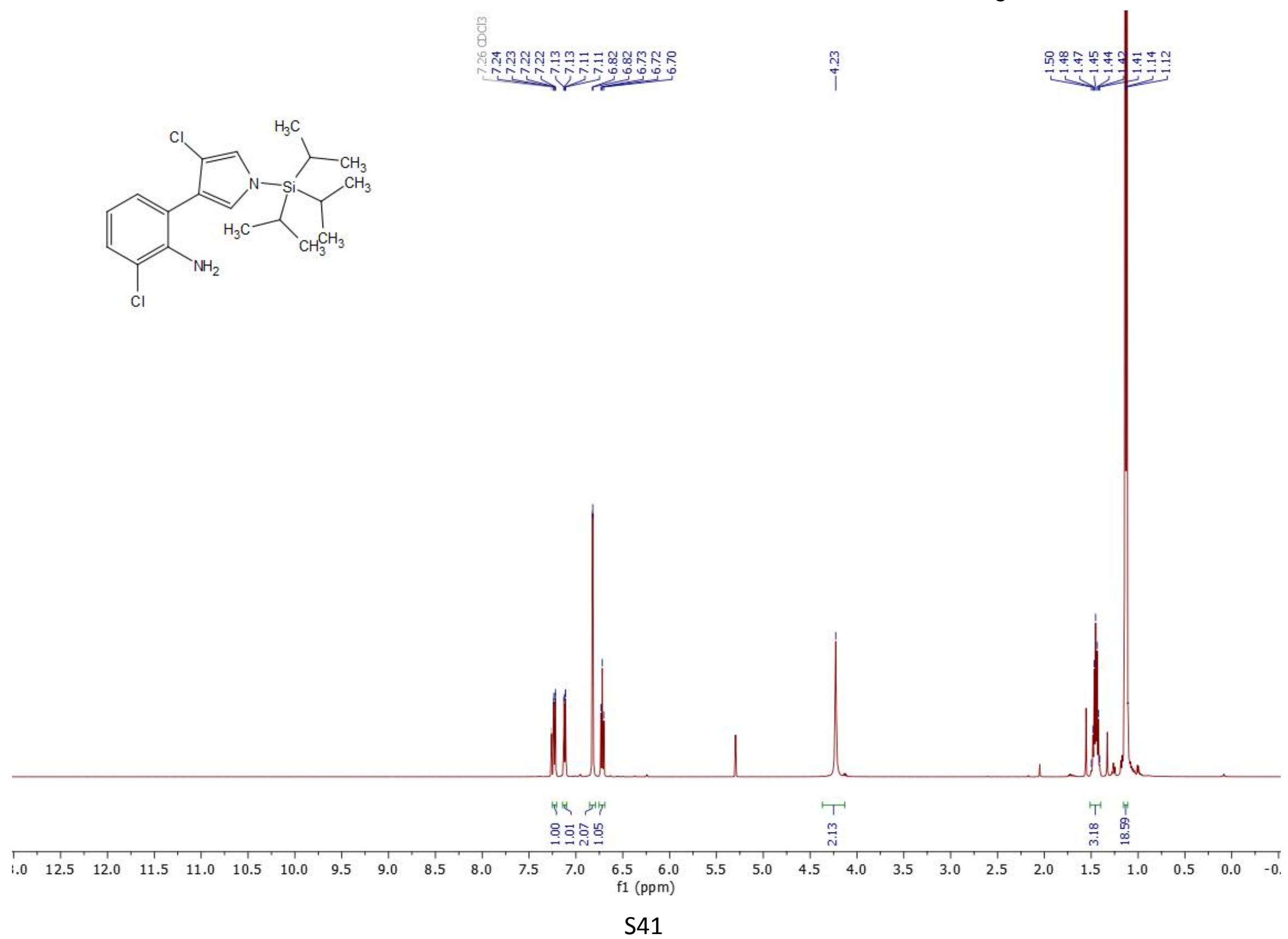
Figure S27: ${ }^{13} \mathrm{C}$ NMR spectrum of $21\left(125 \mathrm{MHz}, \mathrm{CDCl}_{3}\right)$
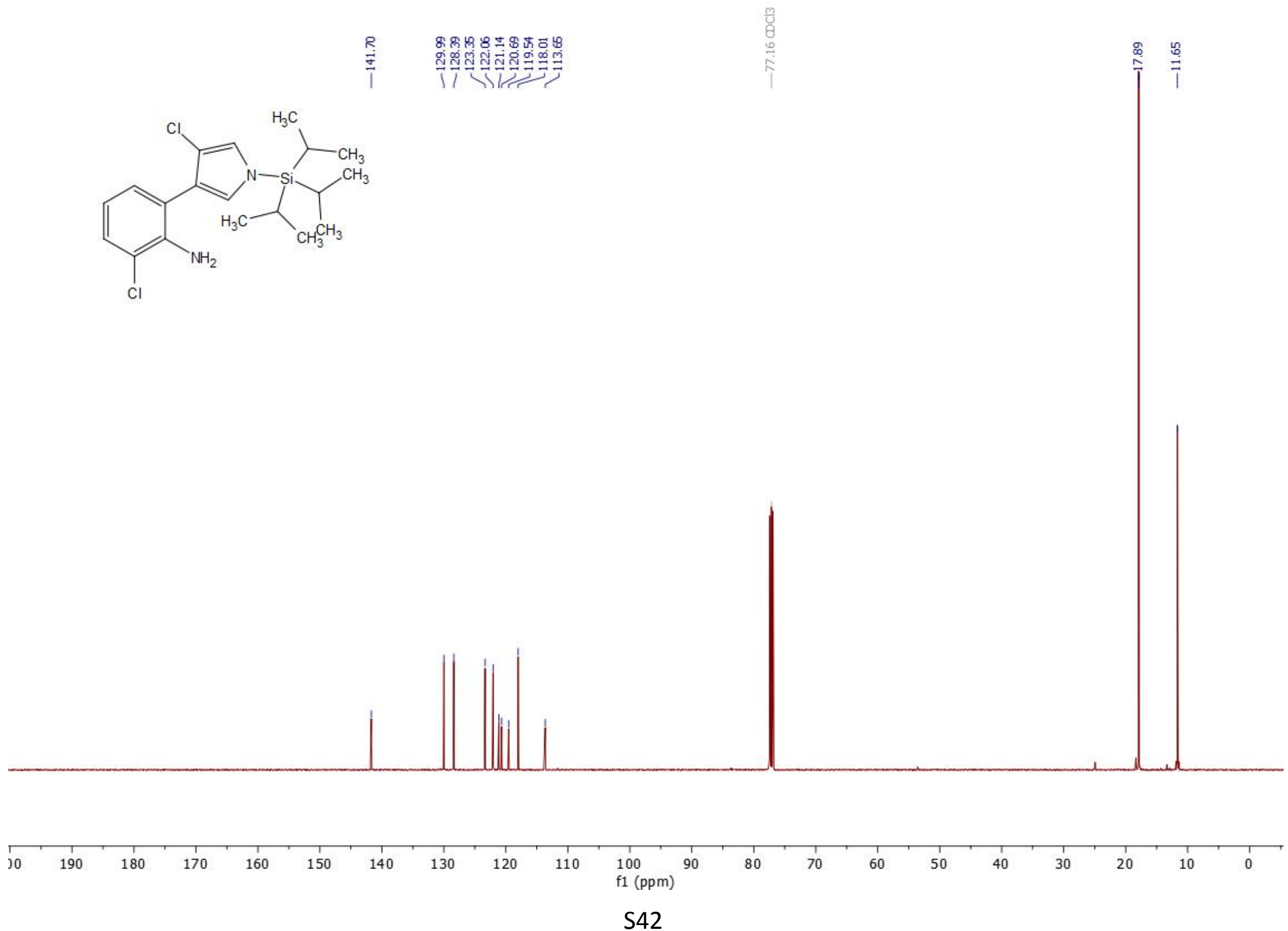
Figure S28: ${ }^{1} \mathrm{H}$ NMR spectrum of $25\left(500 \mathrm{MHz}, \mathrm{CDCl}_{3}\right)$
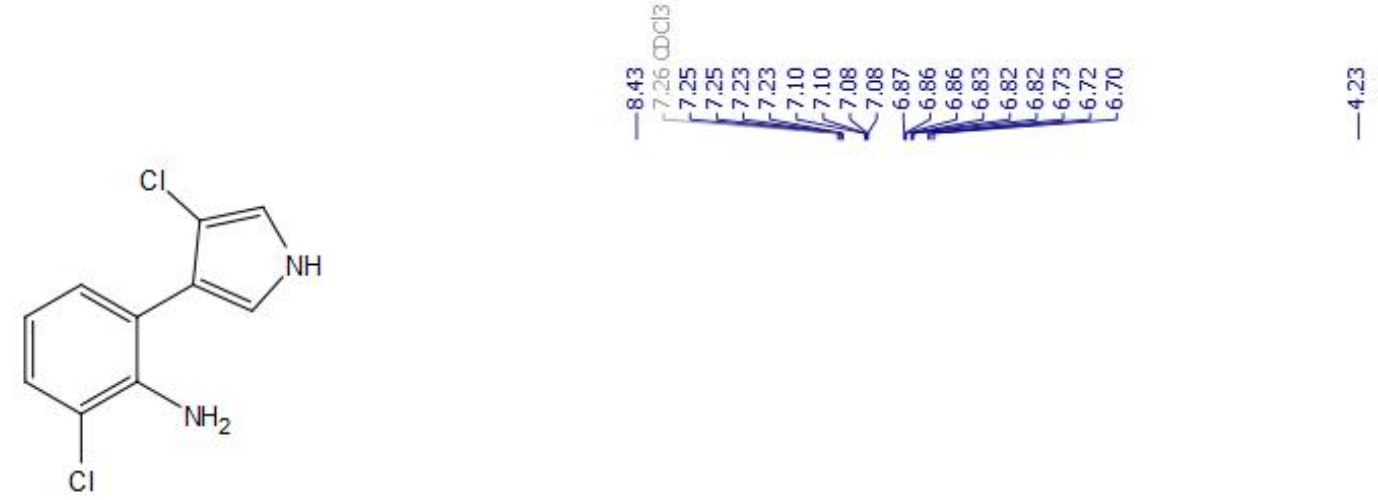

iั

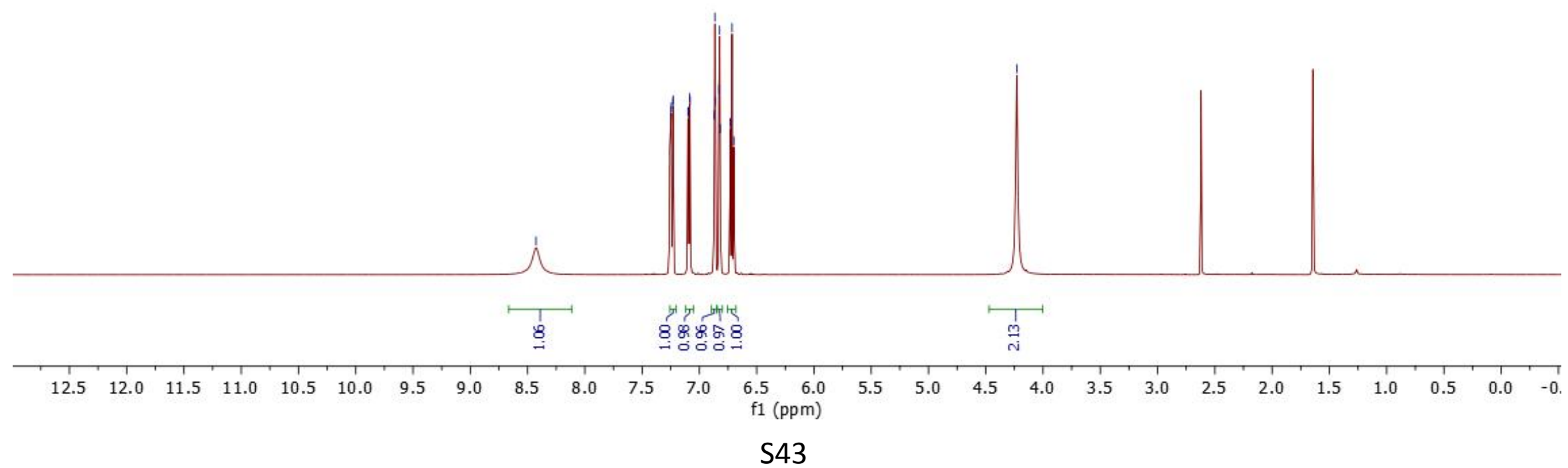


Figure S29: ${ }^{13} \mathrm{C}$ NMR spectrum of $25\left(125 \mathrm{MHz}, \mathrm{CDCl}_{3}\right)$

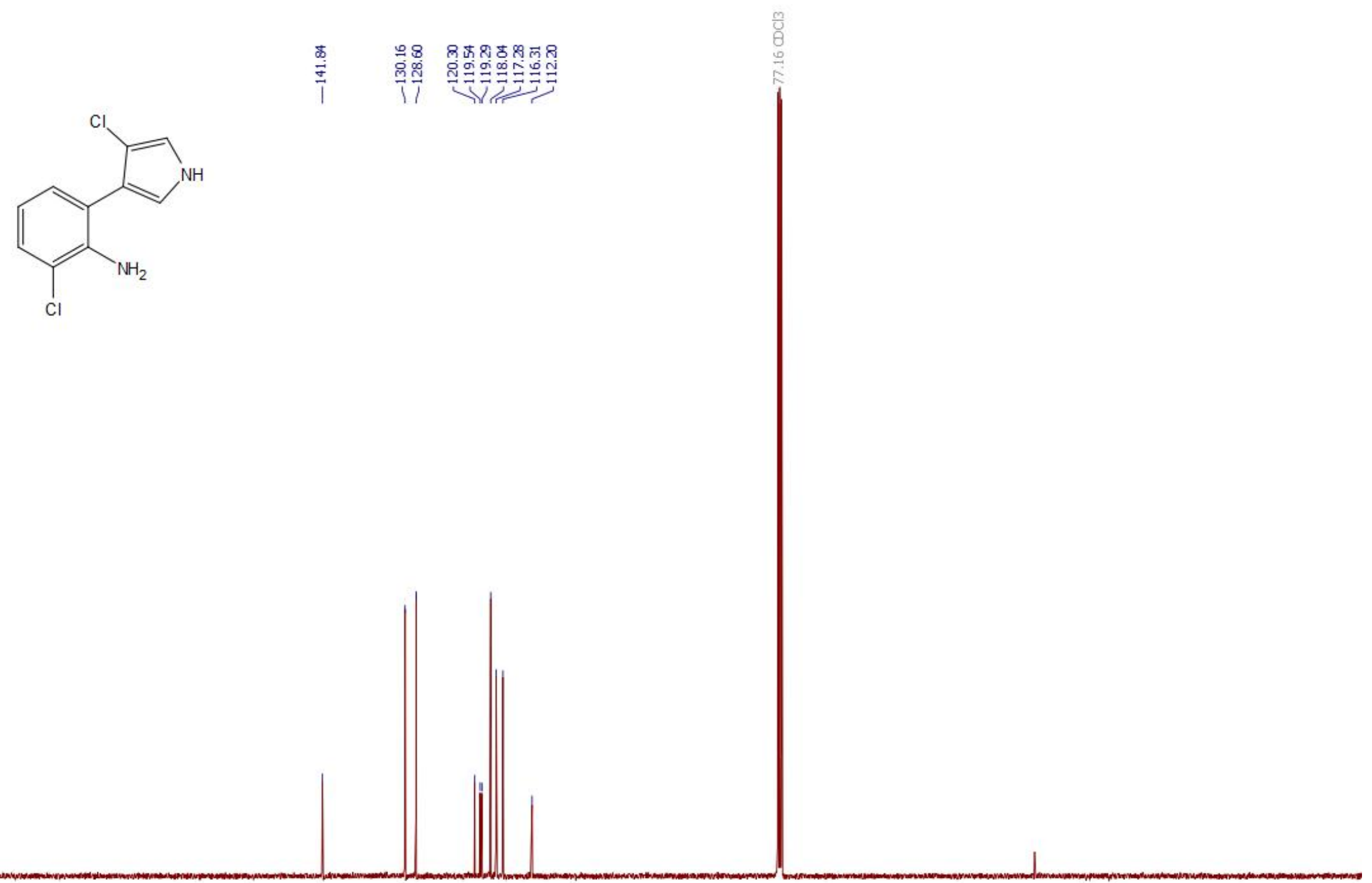


Figure S30: ${ }^{1} \mathrm{H}$ NMR spectrum of pyonitrin $\mathrm{D}\left(500 \mathrm{MHz}\right.$, DMSO- $\left.d_{6}\right)$

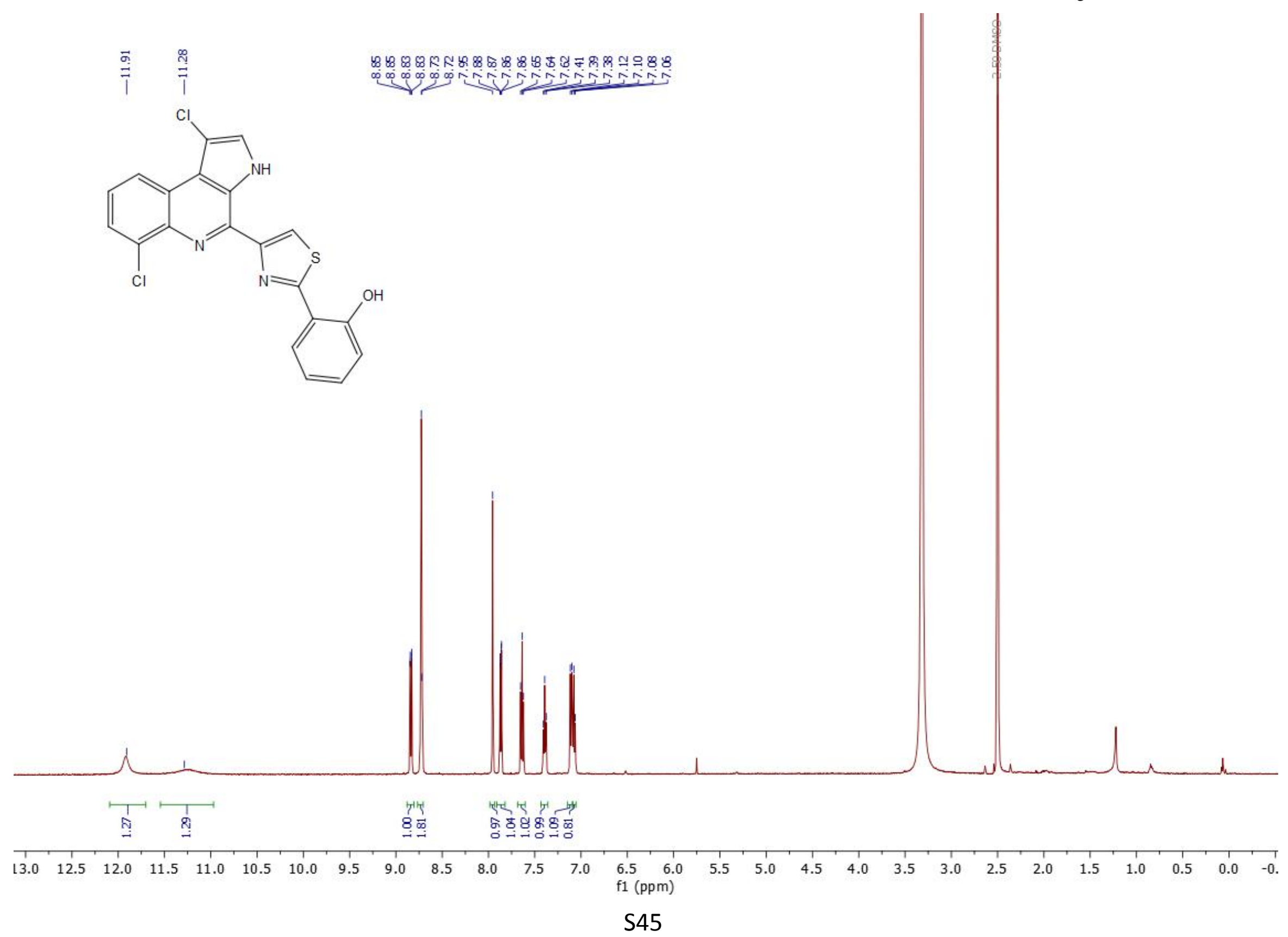


Figure S31: ${ }^{13} \mathrm{C}$ NMR spectrum of pyonitrin D (200 MHz, DMSO- $\left.d_{6}\right)$

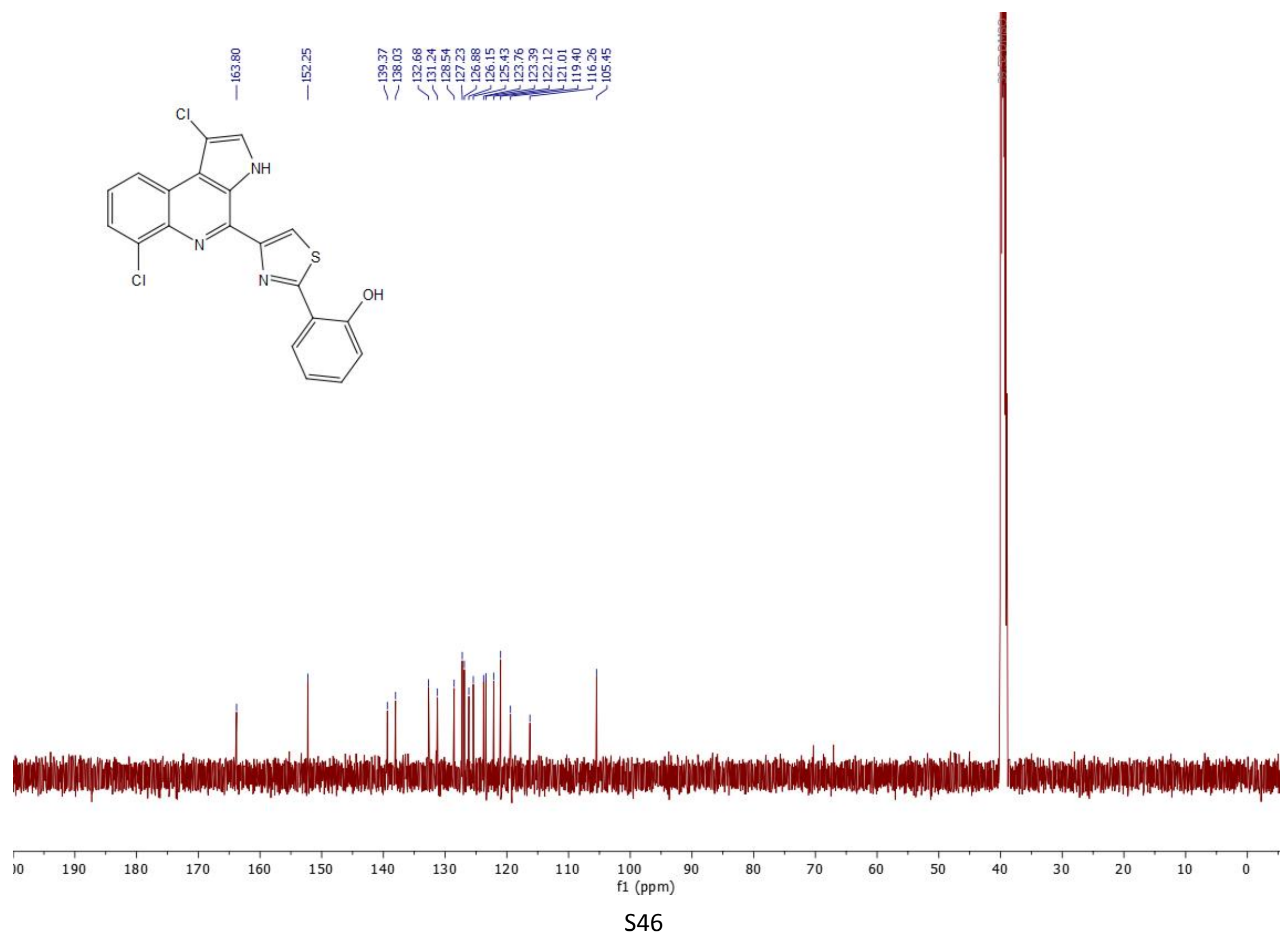


Figure S32: ${ }^{1} \mathrm{H}$ NMR spectrum of $26 a\left(500 \mathrm{MHz}, \mathrm{CDCl}_{3}\right)$
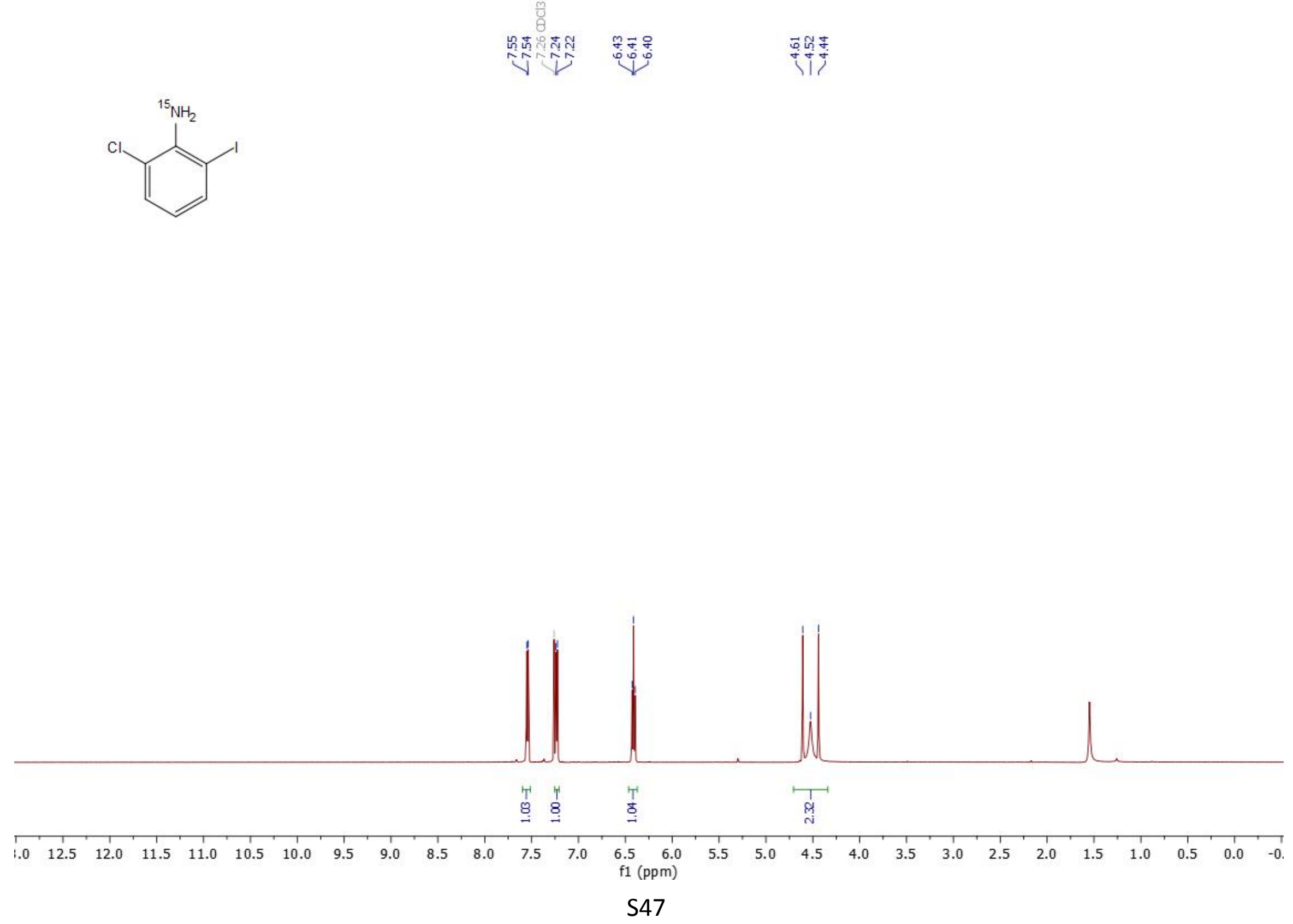
Figure S33: ${ }^{13} \mathrm{C}$ NMR spectrum of $26 a\left(125 \mathrm{MHz}, \mathrm{CDCl}_{3}\right)$<smiles>Sc1c(Cl)cccc1I</smiles>
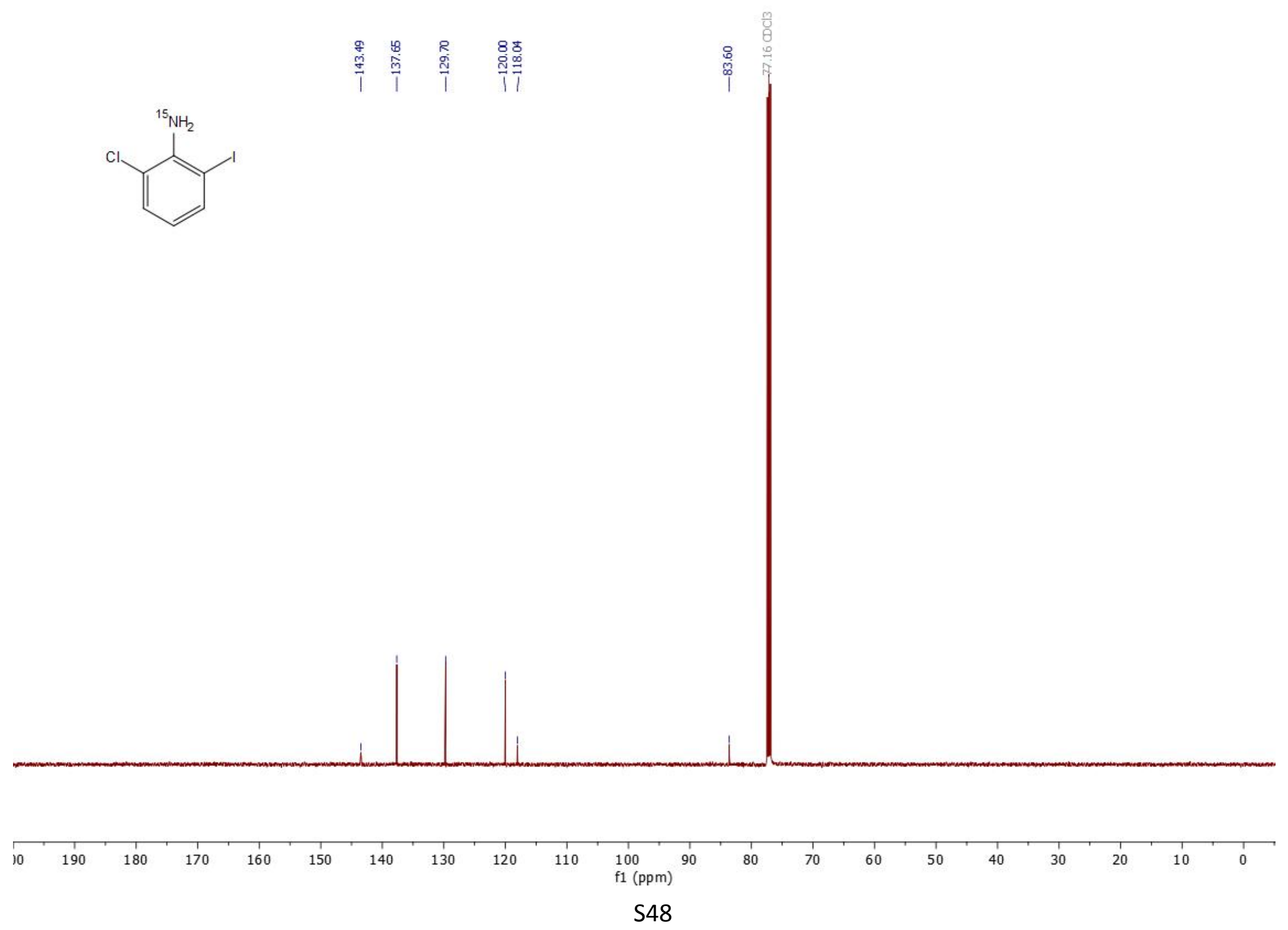
Figure S34: ${ }^{1} \mathrm{H}$ NMR spectrum of $\mathbf{2 6 b}\left(500 \mathrm{MHz}, \mathrm{CDCl}_{3}\right)$

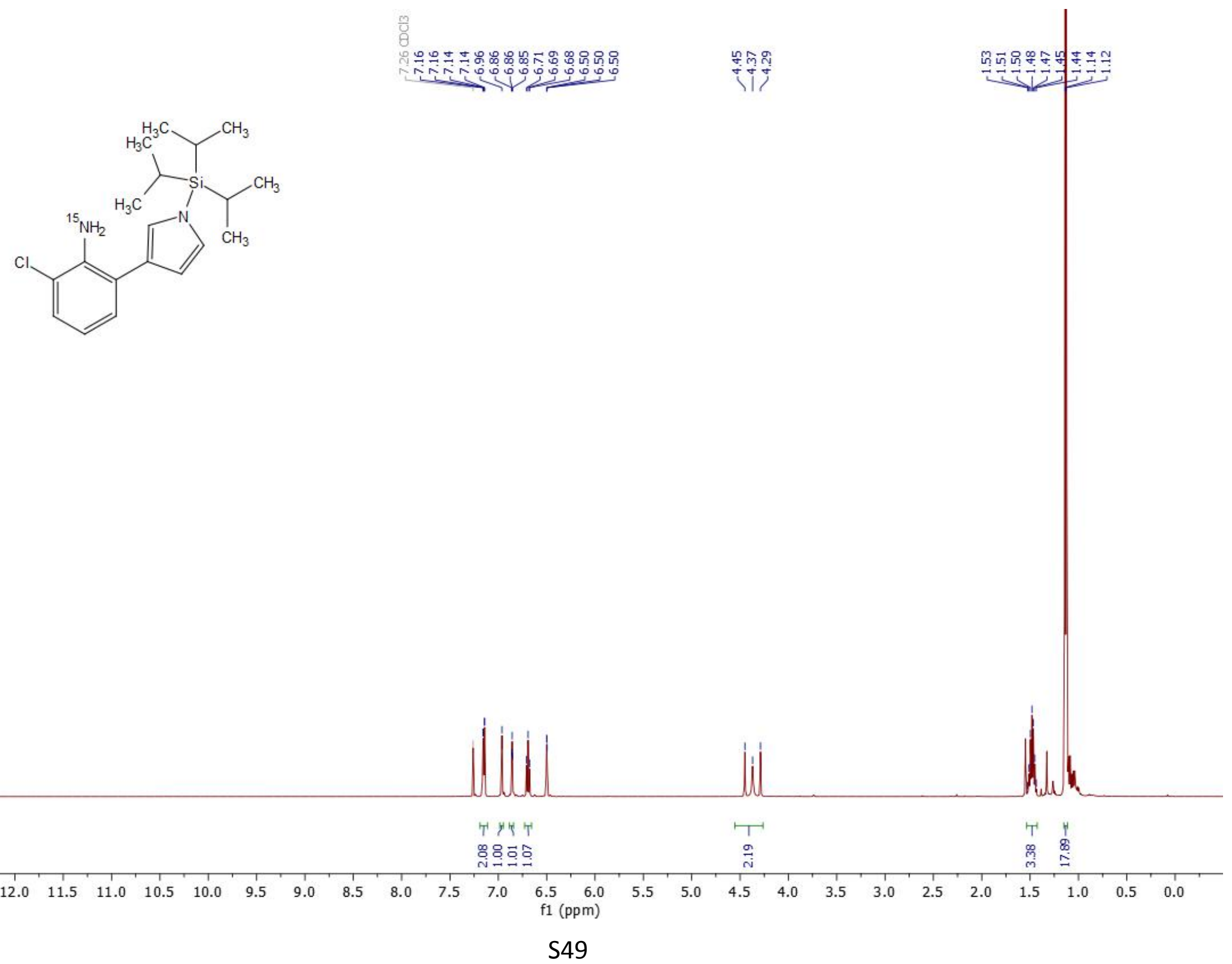


Figure S35: ${ }^{13} \mathrm{C}$ NMR spectrum of $\mathbf{2 6 b}\left(125 \mathrm{MHz}, \mathrm{CDCl}_{3}\right)$

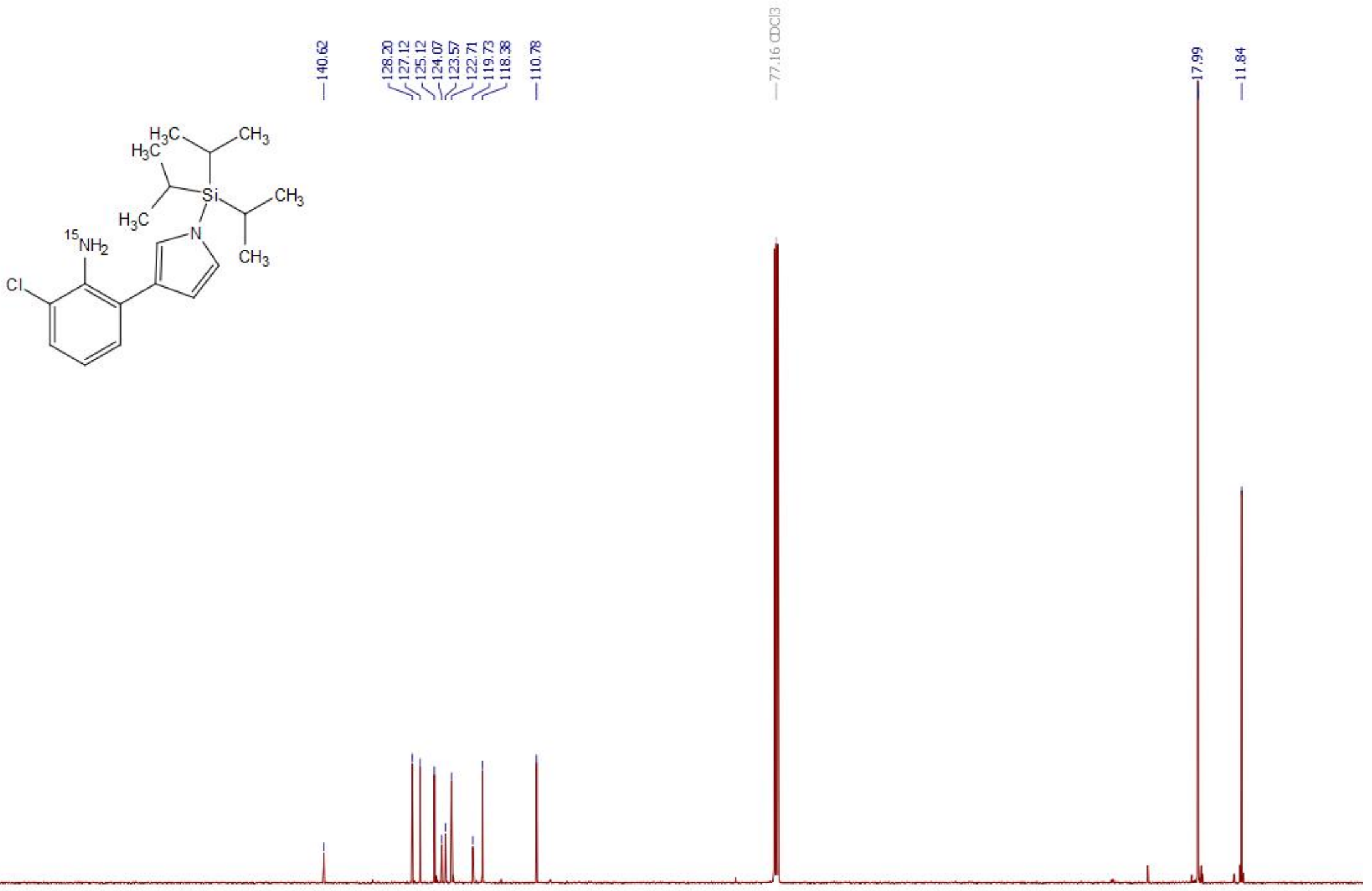


Figure S36: ${ }^{1} \mathrm{H}$ NMR spectrum of $26\left(800 \mathrm{MHz}, \mathrm{DMSO}-d_{6}\right)$

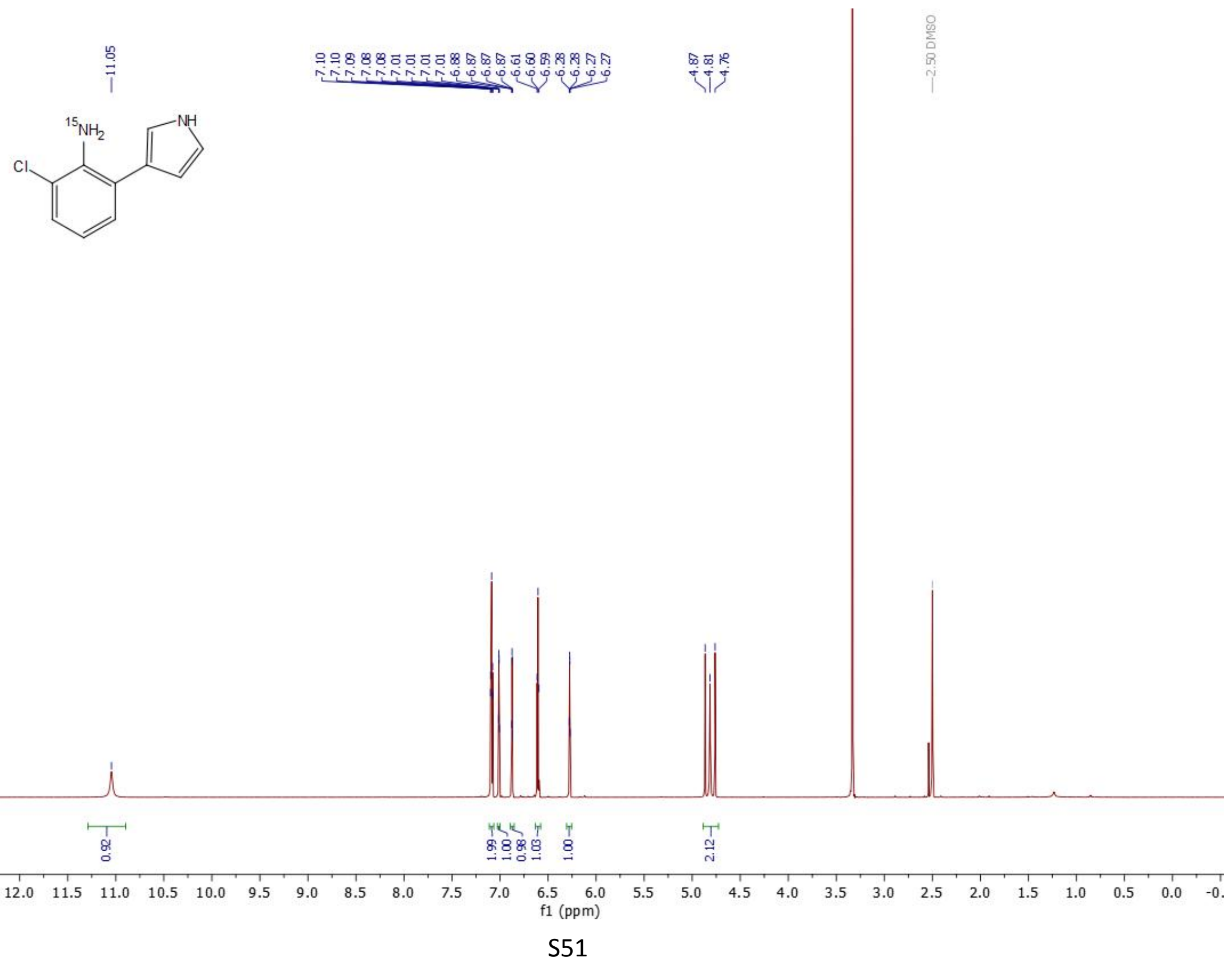


Figure S37: ${ }^{13} \mathrm{C}$ NMR spectrum of $26\left(200 \mathrm{MHz}, \mathrm{DMSO}-d_{6}\right)$

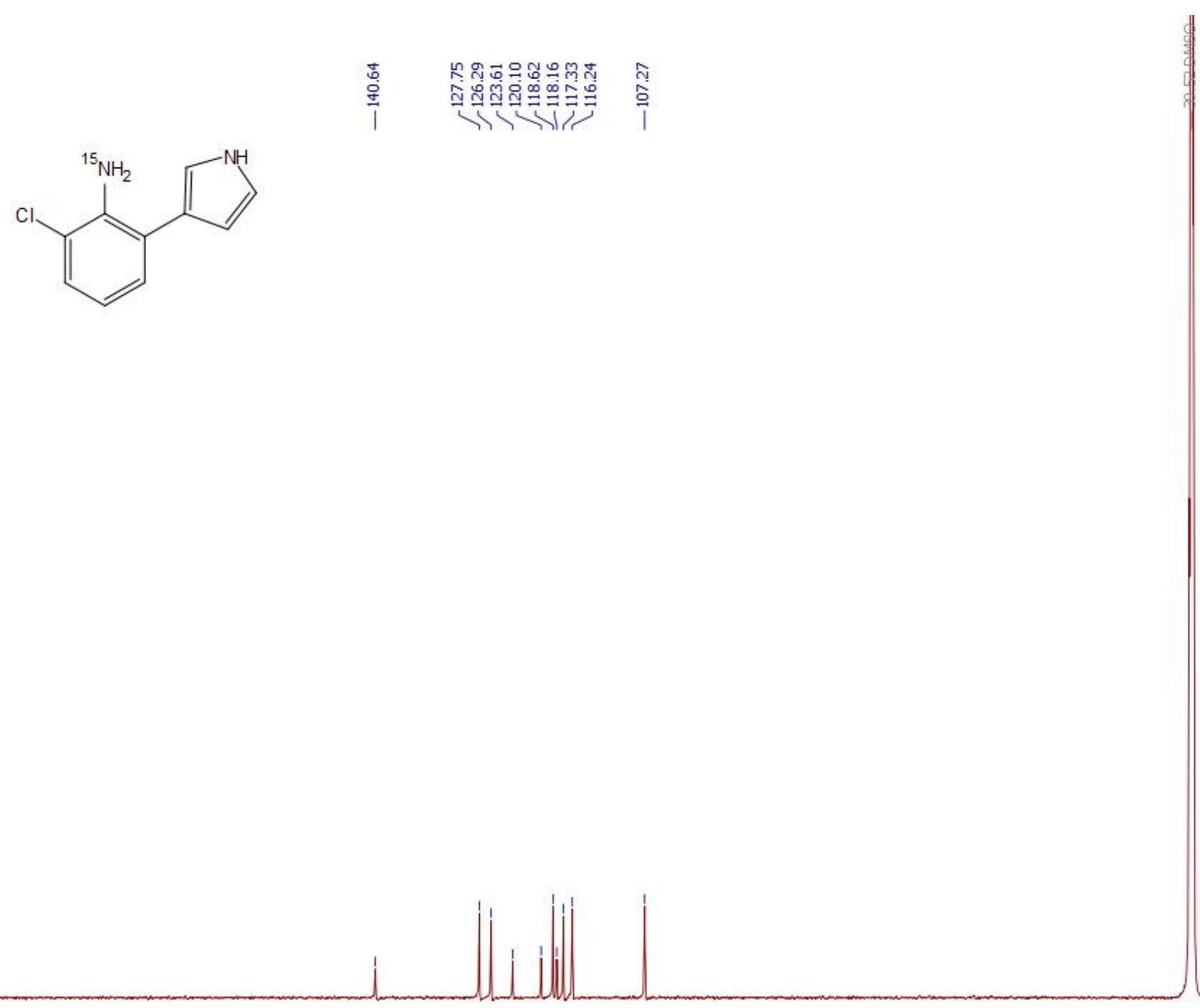

170

160

150

140

130

120

110 
Figure S38: ${ }^{1} \mathrm{H}$ NMR spectrum of ${ }^{15} \mathrm{~N}$ pyonitrin A $29\left(500 \mathrm{MHz}, \mathrm{DMSO}-d_{6}\right)$

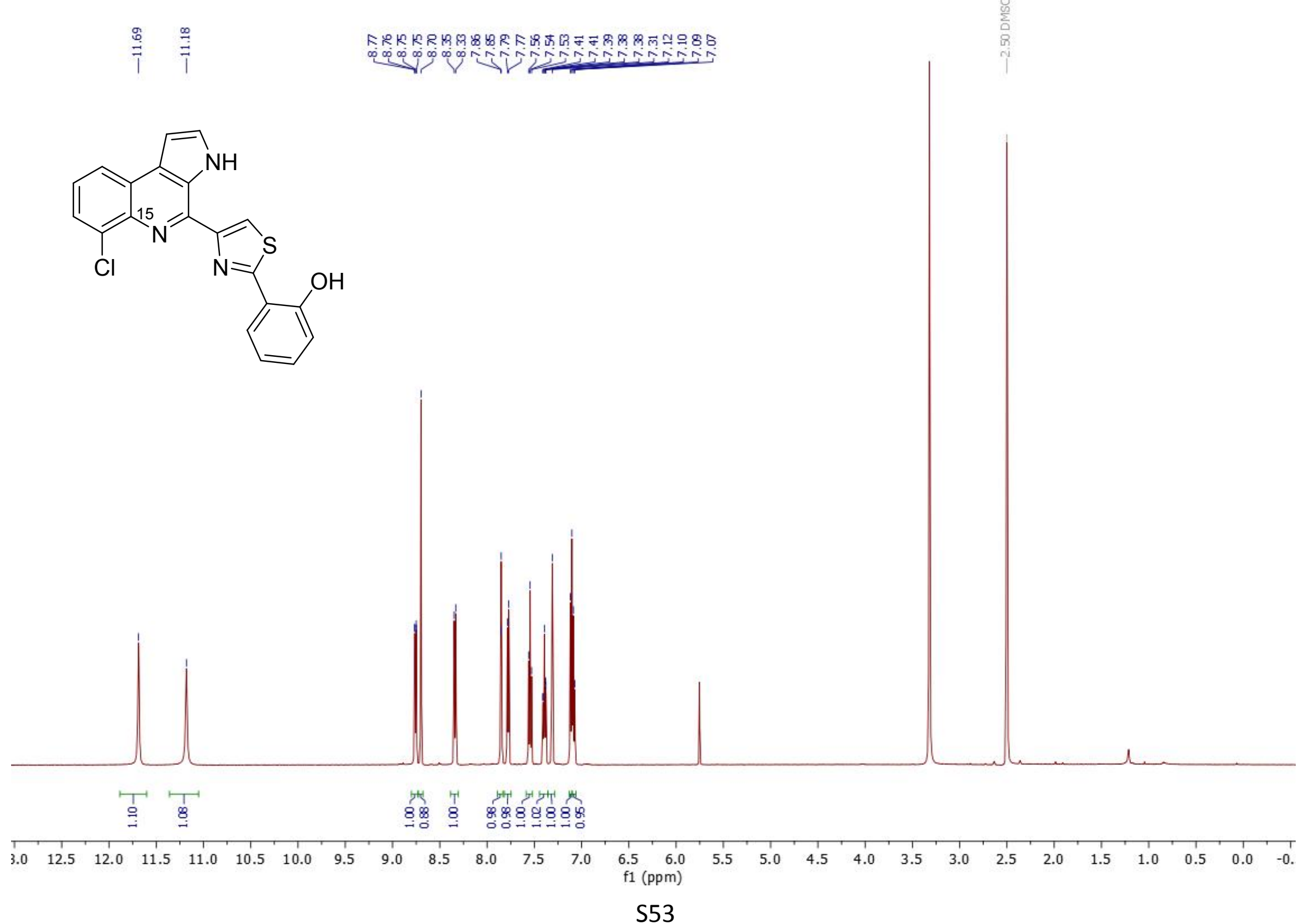


Figure S39: ${ }^{13} \mathrm{C}$ NMR spectrum of ${ }^{15} \mathrm{~N}$ pyonitrin A $29\left(125 \mathrm{MHz}\right.$, DMSO- $\left.d_{6}\right)$

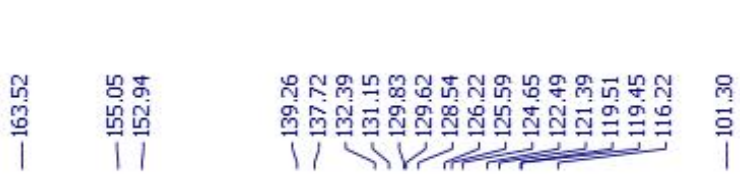<smiles>Oc1ccccc1-c1nc(-c2nc3c(Cl)cccc3c3cc[nH]c23)cs1</smiles>

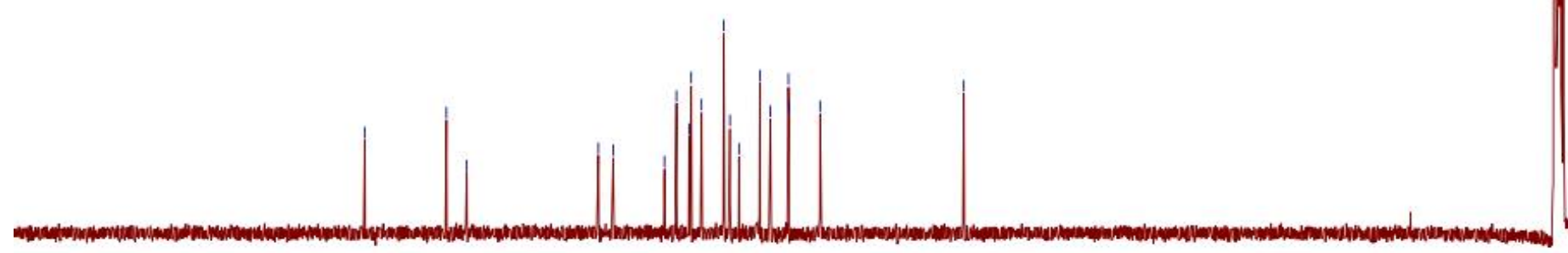


Figure S40: ${ }^{1} \mathrm{H}$ NMR spectrum of $28\left(500 \mathrm{MHz}, \mathrm{DMSO}-d_{6}\right)$

\$8の大ल

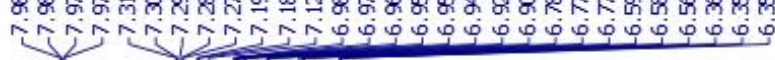
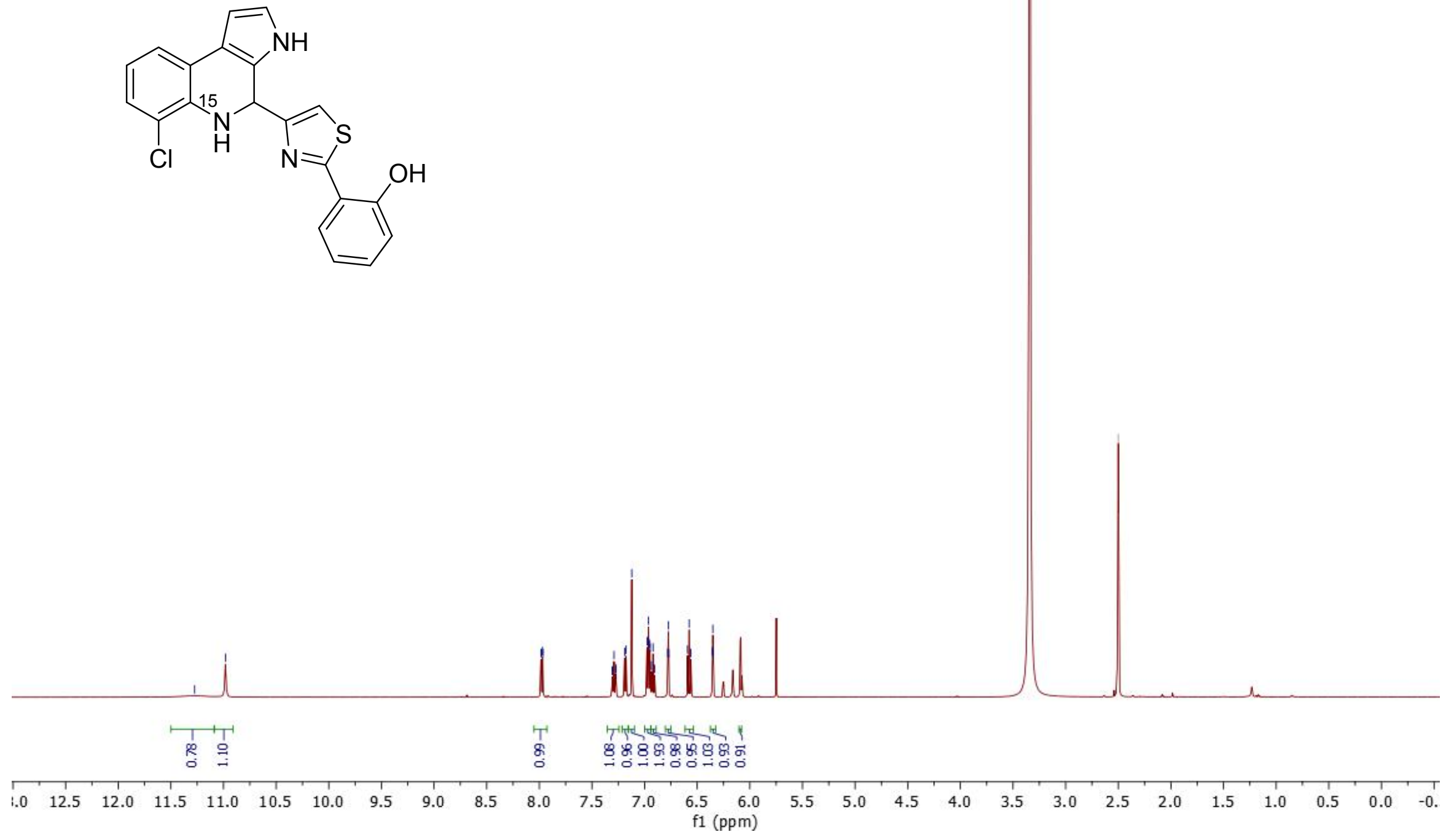
Figure S41: ${ }^{13} \mathrm{C}$ NMR spectrum of $28\left(125 \mathrm{MHz}, \mathrm{DMSO}-d_{6}\right)$

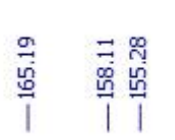

番
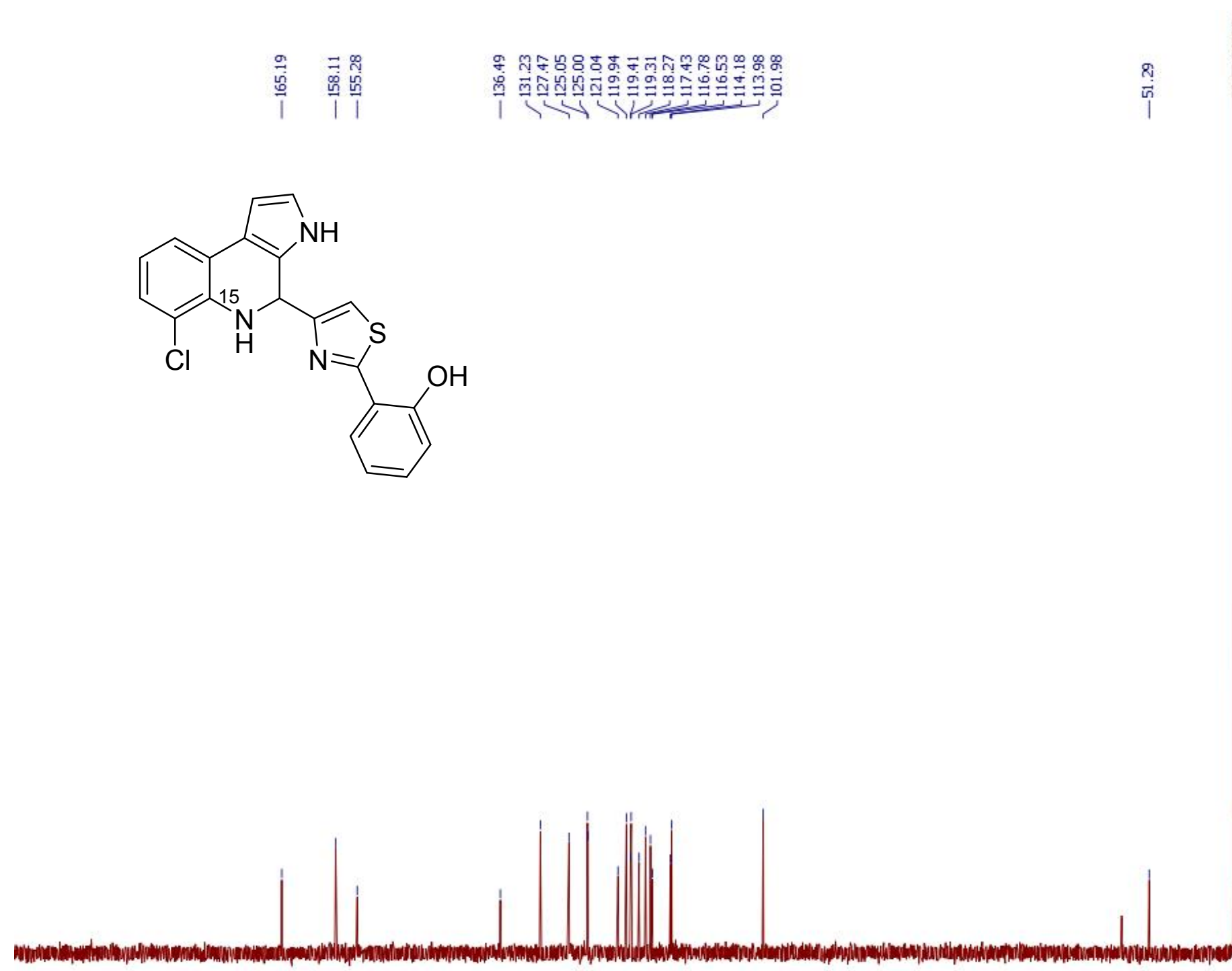

$$
\text { jo }
$$

190

180

170

160

150

140

130

120

110 f1 (ppm)

S56 
Figure S42: ${ }^{1} \mathrm{H}^{15} \mathrm{~N}$ HMBC NMR spectrum of $26\left(500 \mathrm{MHz}\right.$, DMSO- $\left.d_{6}\right)$

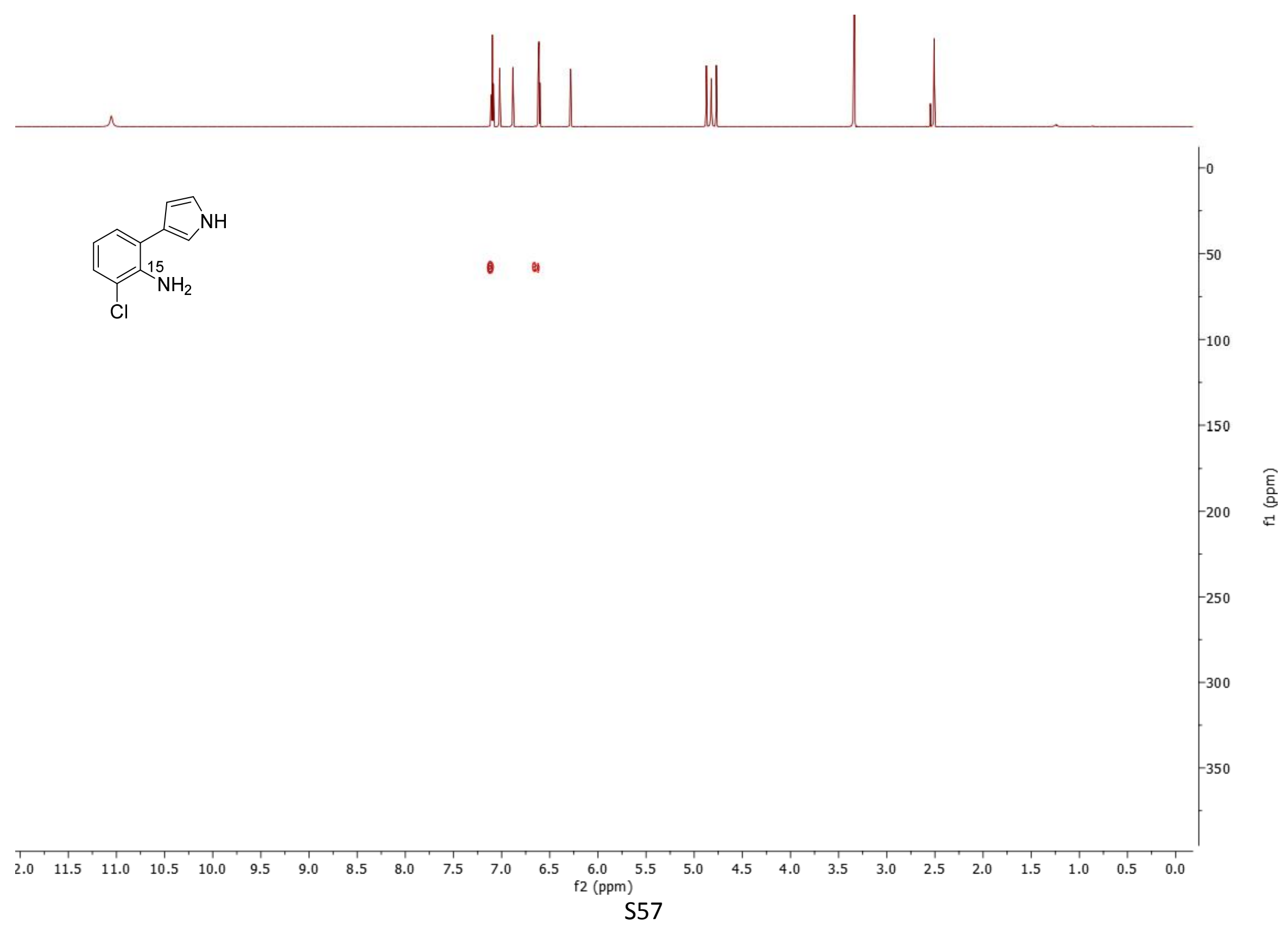


Figure S43: ${ }^{1} \mathrm{H}^{15} \mathrm{~N}$ HMBC NMR spectrum of $28\left(500 \mathrm{MHz}\right.$, DMSO- $\left.d_{6}\right)$

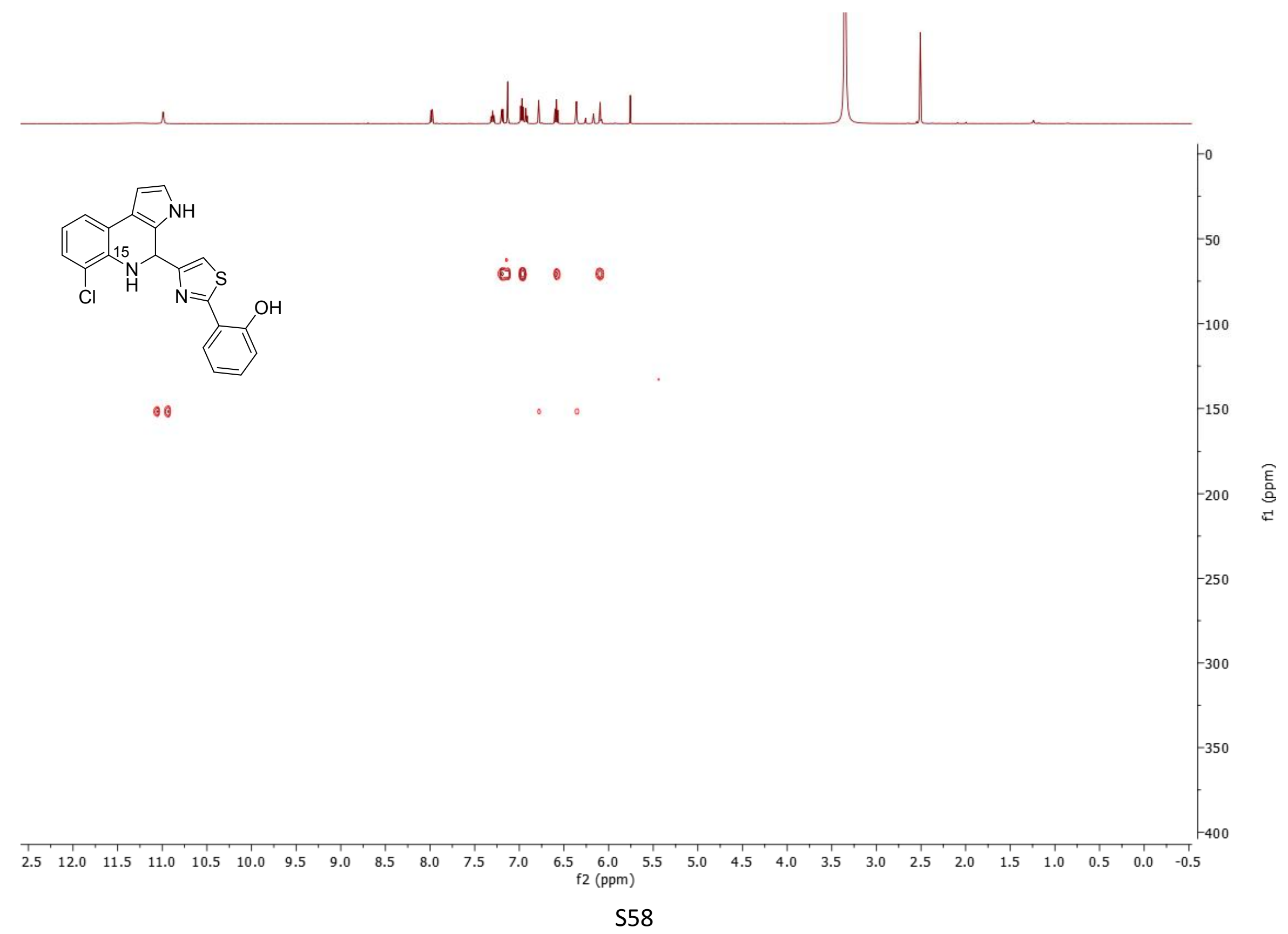


Figure S44: ${ }^{1} \mathrm{H}^{15} \mathrm{~N}$ HMBC NMR spectrum of $29\left(500 \mathrm{MHz}\right.$, DMSO- $\left.d_{6}\right)$

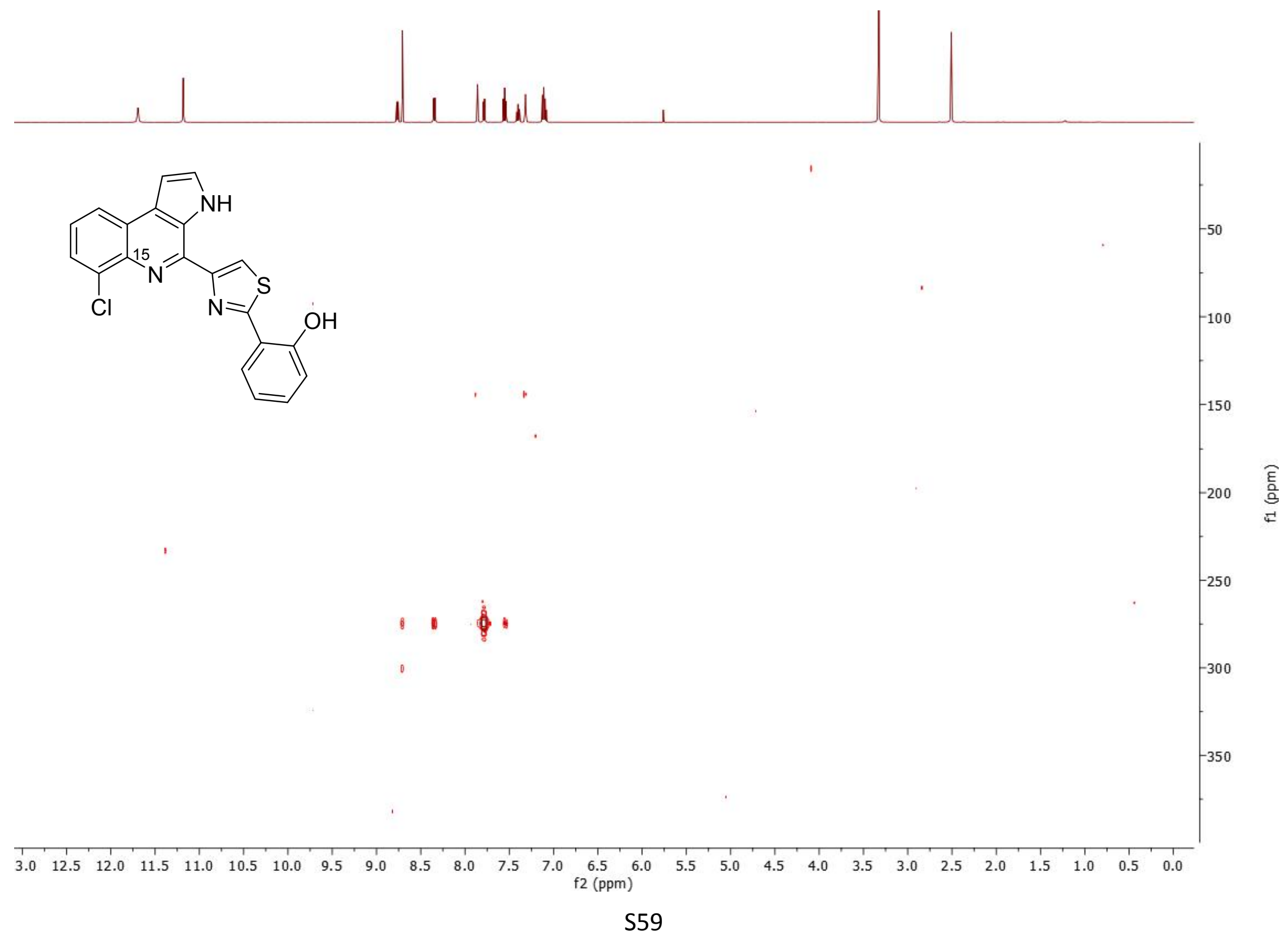


Figure S45: Overlay of the ${ }^{1} \mathrm{H}$ NMR of $26,7,28$ and reaction mixture
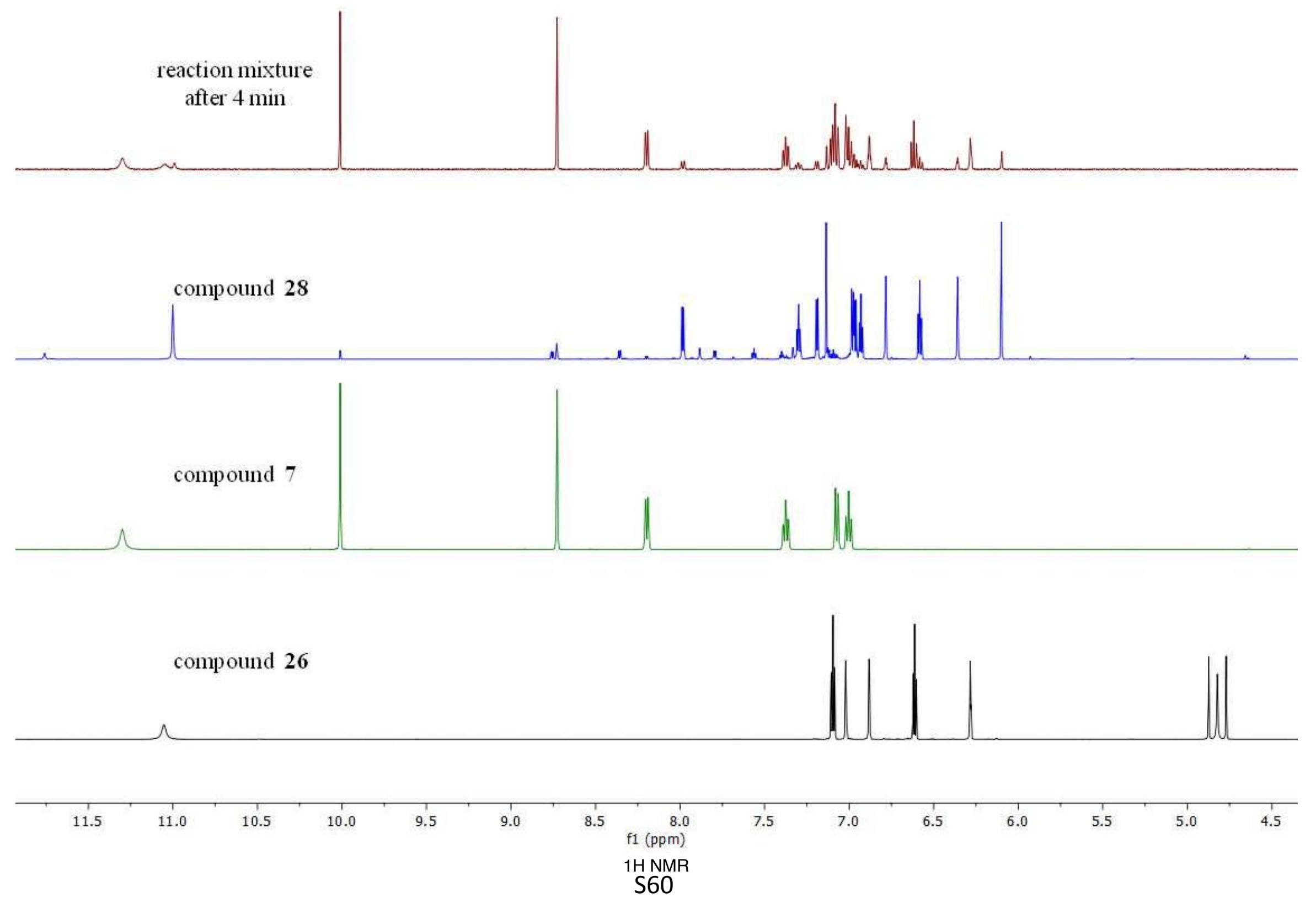